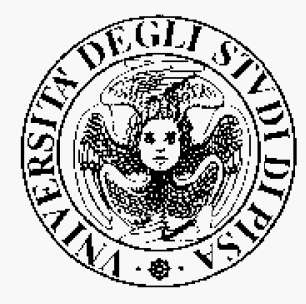

The University of Pisa July, 1998

The faculty of

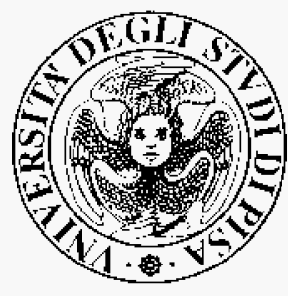

Mathematical, Physical and Natural Science

The dipartement of Physics

Master's Thesis (Tesi di Laurea)

\title{
A study of jet energy measurement at $\mathrm{CDF}$
}

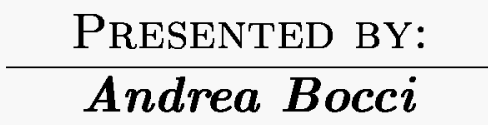

ADVISOR:

Giorgio Bellettini 


\section{Contents}

$\begin{array}{lr}\text { Introduction } & \mathbf{1}\end{array}$

1 QCD and Jets in $p \bar{p}$ collision 3

1.1 Introduction . . . . . . . . . . . . . . . . . . . 3

1.2 QCD: the Standard Theory of Strong Interactions . . . . . . . 4

1.3 QCD Improved Parton Model . . . . . . . . . . . . . . . . . . . . . 5

1.4 Parton Shower and Jet Simulation . . . . . . . . . . . . . . . 9

1.4.1 Parton Branching . . . . . . . . . . . . . . . . . 10

1.5 Hadronization Models . . . . . . . . . . . . . . . . 13

1.5.1 Independent Fragmentation . . . . . . . . . . . 13

1.5.2 String Model . . . . . . . . . . . . . . . . . . 15

1.5.3 Cluster Model . . . . . . . . . . . . . . . . . . . . . . 17

1.6 QCD Events Generators ................ 19

2 Physics Motivations $\quad 20$

2.1 Introduction . . . . . . . . . . . . . . . . . . . 20

2.2 Top Physics at CDF and Run I Experience . . . . . . . . . . . 21

2.2.1 Top Production and Decay Channels . . . . . . . . . 21

2.2 .2 b-Tagging Algorithms . . . . . . . . . . . . . . 22

2.2.3 Semileptonic Channel . . . . . . . . . . . . . 23

2.2.4 All Hadronic Channel . . . . . . . . . . . . . . . . . 27

2.3 Top Quark Mass in Run II . . . . . . . . . . . . . . . . . . . . . . 29

2.4 Light Higgs Physics in Run II . . . . . . . . . . . . . . . . . . 31

2.4.1 $q \bar{q} \rightarrow W H, H \rightarrow b \bar{b} \ldots \ldots . \ldots . \ldots . \ldots 32$

3 The Collider Detector at Fermilab 35

3.1 The Tevatron Collider . . . . . . . . . . . . . . 35

3.2 CDF Detector Overview . . . . . . . . . . . . . . . . 36

3.3 Tracking System . . . . . . . . . . . . . . . . . . . . . . . . . . . 37

3.4 Calorimeters . . . . . . . . . . . . . . . . . . . . . . . . . . . . 39

3.5 Muon Detection . . . . . . . . . . . . . . . 41 
3.6 Trigger . . . . . . . . . . . . . . . . . . . . . . . 42

4 Jets at CDF $\mathbf{4 4}$

4.1 Jet Clustering and Jet Energy Definition . . . . . . . . . . . . 44

4.1.1 JETCLU, the CDF Jet Cluster Algorithm . . . . . . . 44

4.1.2 Jet Energy and Momentum . . . . . . . . . . . . 46

4.2 Jet Energy Corrections . . . . . . . . . . . . . . . . . . . . . . 46

4.2.1 Relative Corrections . . . . . . . . . . . . . . . 47

4.2.2 Absolute Corrections . . . . . . . . . . . . . . . . 48

4.2.3 Underlying Event Subtraction . . . . . . . . . . 50

4.2.4 Out-of-Cone Addition . . . . . . . . . . . . . . . . 50

4.2.5 Specific Corrections . . . . . . . . . . . . 51

5 Particle Level Study and Jet Algorithm 53

5.1 Introduction . . . . . . . . . . . . . . . . 53

5.2 Physics Effects . . . . . . . . . . . . . . . . 55

5.2.1 Reconstruction method and Sample Selection . . . . . 57

5.3 Out Of Cone Corrections . . . . . . . . . . . . . . . . . . 58

$5.4 K_{T}$ Algorithm . . . . . . . . . . . . . . . 61

5.4 .1 The $K_{T}$ Algorithm . . . . . . . . . . . . . . . . 61

5.4.2 Comparison of $K_{T}$ and Cone Algorithms . . . . . . . 63

5.4.3 Variation of $\mathrm{K}_{T}$ Algorithm Parameters . . . . . . . . . 63

5.5 Merging In Extra Jets With The Cone Algorithm . . . . . . . 65

5.5.1 A More Sophisticated Method of Merging Jets . . . . . 68

5.6 The ABC Jet Algorithm . . . . . . . . . . . . . . . . . . . 70

5.7 Applying Extra Jet Cuts . . . . . . . . . . . . . . . . 71

5.8 Conclusions . . . . . . . . . . . . . . . . . 72

6 Detector Resolution Study: Method $\quad 73$

6.1 Introduction . . . . . . . . . . . . . . . . . 73

6.2 Preliminary Study Using CTC . . . . . . . . . . . . . . . 74

6.3 Classification Method . . . . . . . . . . . . . . . 78

6.4 Classification Method: An Example . . . . . . . . . . . . 86

6.5 How Many "Golden" Towers Are There? . . . . . . . . . . . . 89

6.6 New definition of tower energy . . . . . . . . . . . . . . . 90

7 Detector Resolution Study: Results $\quad 94$

7.1 The "Photon + Jet" Sample . . . . . . . . . . . . . . . . 94

7.2 Event Selection . . . . . . . . . . . . . . . . . . 95

7.3 Jet Momentum Balancing . . . . . . . . . . . . . . . 97

7.3.1 Data Sample Selection Cuts . . . . . . . . . 97 
7.3.2 "Classification" vs. "JTC96" . . . . . . . . . . . . . 98

7.4 "Dijet-like" $k_{t}$ Balancing . . . . . . . . . . . . . . . . . . 99

7.4.1 "Dijet-like" $k_{t}$ Description . . . . . . . . . . . . . . . 99

7.4 .2 Origin of the $k_{t}$ Components . . . . . . . . . . . . 102

7.4.3 Central Detector Resolution . . . . . . . . . . . . . 103

7.4.4 "Classification" vs. "JTC96" . . . . . . . . . . . . 105

$\begin{array}{ll}\text { Conclusions } & 108\end{array}$

$\begin{array}{ll}\text { Acknowledgements } & 109\end{array}$

$\begin{array}{ll}\text { Bibliography } & 111\end{array}$ 


\section{List of Tables}

1.1 Elementary point-like particles in the Standard Model . . . . 5 5

2.1 Subsamples of $W+\geq 4$ jet events which are used for the top quark mass measurement . . . . . . . . . . . . 25

2.2 Systematic uncertainties on the top mass measurement in the semileptonic channel . . . . . . . . . . . . . . 28

2.3 Systematic uncertainties on the top mass measurements in all hadronic channel . . . . . . . . . . . . . . . . . . . . 29

3.1 Parameters of the CDF tracking detectors . . . . . . . . 39

3.2 Angular coverage and resolution of the CDF calorimeters . . . 42

4.1 Underlying Event and Out-Of-Cone corrections . . . . . . . 51

4.2 Fitted parameters of AA corrections . . . . . . . . 52

5.1 Di-jet mass resolution for the three different cone sizes. . . . . 59

5.2 Out-of-cone corrections for the three different cone sizes . . . 61

6.1 Results of PYTHIA $\gamma+$ jet simulation. ISR turned off. . . . . 77

6.2 Tower classification for "Photon + Jet" data sample. . . . . . 89

6.3 Summary of new tower energy definition . . . . . . . . . 93

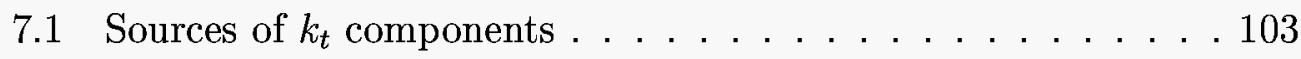




\section{List of Figures}

1.1 Sketch of a hard proton-antiproton interaction $\ldots \ldots \ldots$

1.2 Parton distribution functions . . . . . . . . . . . . 8

1.3 Parton branching of a) incoming parton (spacelike branching)

and $b$ ) outgoing parton (timelike branching) . . . . . . 11

1.4 Initial state branching . . . . . . . . . . . . . . 11

1.5 Representation of parton branching by path in $(\mathrm{t}, \mathrm{x})$-space $\ldots 12$

1.6 Parton shower in timelike evolution branching . . . . . . . 13

1.7 Hierarchy of mesons in independent fragmentation . . . . . . 14

1.8 Working mechanism in the string model . . . . . . . . 16

1.9 Parton shower in the string hadronization model . . . . . . . 17

1.10 Parton shower in the string hadronization model, before final state clustering into hadrons. . . . . . . . . . . 18

2.1 Top quark production: a) dilepton channel, b) all hadronic channel . . . . . . . . . . . . . . . . . . 22

2.2 Top production: a) semileptonic channel, b) direct $\mathrm{W}+$ multijets 22

2.3 Reconstructed mass distribution of the combined four subsamples . . . . . . . . . . . . . . . . 26

2.4 Top mass reconstruction in all hadronic channel . . . . . . . 29

2.5 The CDF top mass values in each decay channel . . . . . . . 30

2.6 CDF measurement of $M_{W}$ and $M_{t o p}$ and Higgs mass prediction 30

2.7 Production of Higgs boson in the process WH with $H \rightarrow b \bar{b}$. . 32

2.8 Expected signal+background mass distribution for the WH process with $10 \mathrm{fb}^{-1}$ of data at $2 \mathrm{TeV} \ldots \ldots \ldots 33$

2.9 Expected signal+background mass distribution for the $\mathrm{WH}$ process with $10 \mathrm{fb}^{-1}$ of data at $2 \mathrm{TeV} \ldots \ldots \ldots . \ldots 34$

3.1 Overall view of the Tevatron Collider . . . . . . . . . . 36

3.2 Overall view of CDF detection system . . . . . . . . 37

3.3 Coverage of the calorimeter towers in $\eta-\phi$ space $\ldots \ldots .40$

4.1 Relative correction for Run 1B . . . . . . . . . . . . 49 
4.2 Absolute corrections: Behrends curves. . . . . . . . . . . . 50

5.1 The $W \rightarrow q \bar{q}$ mass distribution with pure algorithm effects (solid), and pure detector effects (dashed) . . . . . . . . . 54

5.2 The di-jet invariant mass distribution without final state radiation ........................ 55

5.3 The di-jet invariant mass for cone $0.4 \ldots \ldots 6$

5.4 Minimum $W$ energy fraction of the two leading jets for the reconstructed events with mass $>100 \mathrm{GeV}$. . . . . . . . . 57

5.5 Invariant mass distribution using a cone 0.4 for PYTHIA and HERWIG . . . . . . . . . . . . . . . . . . 57

5.6 An example of the Paw-based event display . . . . . . . . . . 59

5.7 Di-jet invariant mass for cone radius 0.4 (solid), 0.7 (dashed), and 1.0 (dotted) . . . . . . . . . . . . . . . . . 60

5.8 The distribution of the difference $\mathrm{E}_{T}$ parton $-\mathrm{E}_{T}$ jet for cone radius 0.4 and for four different slices of $\mathrm{E}_{T}$ jet . . . . . . . . 60

5.9 The invariant mass distribution with some out-of-cone corrections and different cone sizes . . . . . . . . . . . . . . 61

5.10 Comparison of $\mathrm{K}_{T}$ and Cone algorithms . . . . . . . . . . 63

5.11 Comparison of various resolution parameters for the $K_{T}$ and Cone algorithms . . . . . . . . . . . . . . . . . 64

5.12 Comparison of different merging schemes within the $\mathrm{K}_{T}$ algorithm . . . . . . . . . . . . . . . . . . . 64

5.13 The 2-jet and 3-jet mass distributions for jets coming and not coming from $W \ldots \ldots \ldots . \ldots . \ldots 65$

5.14 The fraction of energy from the $W$ for each of the first three jets 66

5.15 The $\Delta R$ separation between the third jet and the closest leading jet . . . . . . . . . . . . . . 66 66

5.16 Comparison of the mass distributions for the cone 1.0 and the cone 0.4 algorithm . . . . . . . . . . . . . . . . . . . 67

5.17 Test-case signal versus background example . . . . . . . . . 68

5.18 Jet energy fraction of the extra jet closest to the second jet . . 69

5.19 Sophisticated merging compared to simple merging . . . . . . 69

5.20 Invariant mass distribution from the $\mathrm{ABC}$ algorithm . . . . . . 70

5.21 Percentage of events in the tails of the mass distribution versus the third jet cut efficiency . . . . . . . . . . . . . 71

6.1 Schematic view of the calorimeter region near the impact point of a track . . . . . . . . . . . . . . . . . . . . . . . . . . . . 79

6.2 Tracks momentum distribution for $\gamma+$ jet data sample events 80 
6.3 a) Relative $\phi$ position distribution of tracks inside target tower.

b) Calorimeter energy (in $3 \times 1$ window) versus the momentum fraction distribution . . . . . . . . . . . . . . . . . . 81

6.4 Calorimeter response to a photon of $4 \mathrm{GeV}$ in the central region as a function of $z$ position in the wedge . . . . . . . . 82

6.5 a) CEM tower response as a function of hit point. b) CEM tower response as a function of photon energy. . . . . . . . . . 83

6.6 $\Delta z=z_{\text {track }}-z_{C E S}$ distribution when cut on CEM energy is or is not applied . . . . . . . . . . . . . . . . . . 86

6.7 Lego plot and side view of the calorimeter . . . . . . . . . 87

6.8 CTC view of the same event . . . . . . . . . . . . . 87

6.9 a) View of the central calorimeter of a Monte Carlo event. b) Same view after the classification method is applied . . . . . 88

$6.10\langle C E M\rangle_{c h}-C E M$ distribution for "track tower" . . . . . . . . 91

6.11 Photon-Jet balancing using raw (CEM+HAD) energy for the jet . . . . . . . . . . . . . . . . 93

7.1 Leading order diagrams for photon production in $p \bar{p}$ collisions. 95

7.2 Momentum balancing width $\sigma_{B}$ distribution as function of $P_{T}^{\gamma} \quad 98$

$7.3 k_{t}$ example: schematic view of a "Photon + Jet" event . . . . 99

$7.4 k_{t}$ example: jet and photon exactly and not back-to-back . . . 100

7.5 Width of $k_{t_{\xi}}$ distribution (a) and of $k_{t_{\eta}}$ distribution (b) as a function of $\sqrt{E_{t}^{\gamma}}$. . . . . . . . . . . . . . 104

7.6 Effective detector resolution $\sigma_{D}=\sqrt{\sigma_{\xi}^{2}-\sigma_{\eta}^{2}}$ distribution as function of $\sqrt{E_{t}^{\gamma}} \ldots \ldots \ldots 6$

7.7 The value of $\sigma_{\xi}(\mathrm{a})$ and $\sigma_{\eta}(\mathrm{b})$ as function of $\sqrt{E_{T}^{\gamma}}$ for the two methods . . . . . . . . . . . . . . . . . . . 107

7.8 The value of $\sigma_{D}$ adopting the two methods as a function of $\sqrt{E_{T}^{\gamma} \ldots \ldots \ldots \ldots \ldots \ldots} 107$ 


\section{Introduction}

This thesis describes the effort being made to improve the Jet Energy Reconstruction as performed by the CDF international collaboration at the Tevatron collider.

This experiment studies proton-antiproton interactions at a center of mass energy of $1.8 \mathrm{TeV}$. During the three years data taking period - Run I, from 1992 to 1995 - the CDF experiment collected an amount of data correspond-

ing to a total integrated luminosity of $110 \mathrm{pb}^{-1}$. One of the major results obtained analyzing this data sample is the discovery of the top quark.

In the year 2000 a new period of data taking, Run II, will start with a higher luminosity and a slightly higher center of mass energy giving us the chance to explore high energy physics even deeper. In preparation of this new run several upgrades are being made to adapt the CDF detector to the high luminosity foreseen and to improve its capabilities.

Many signatures requested to trigger the detector aim at signaling a quark or a gluon in the final state. Unfortunately we are not able to measure quarks as free particles because they undergo a fragmentation process when turning into jets of particles. Thus it is of key importance to build up algorithms which reconstruct the energy of the initial parton starting from the jet informations. The description of the algorithm adopted till now will be given as an introduction to the new method being developed, that will be the main subject of this thesis.

In Chapter 1 we will give a theoretical introduction on strong interactions to describe the mechanism to produce hadronic jets.

In Chapter 2 we will describe some results from the experiment where the reconstruction of hadronic jets was important. Here we will also mention some important results which we think we can obtain during new the data taking period. We will give particular emphasis to those processes where an improved jet energy measured would bring to better results.

In Chapter 3 we will give a description of the CDF detector including some more details on the elements which are relevant for jet energy reconstruction.

The way of defining jets which has been used by CDF so far, will be the 
subject of chapter 4 .

Starting from the present CDF algorithm we studied the various problems which arise with jet reconstruction. Those problems can be grouped into two categories, the one including effects coming from physics and a second one including the effects due to a non-perfect resolution of our detector.

In Chapter 5 the physics effects limiting jet energy reconstruction will be addressed. We will discuss the radiation of hard gluons both from initial state and final state partons and we will show how these problems are connected with jet definition algorithms.

In Chapter 6 we will describe a new method to define jet energy making use of some detector informations which are not used in the present algorithm. The energy of each single calorimeter tower will be re-defined taking into account not only the energy released in the calorimeters, but also the informations on the shower development through it and the tracking informations coming from the Central Tracking Chamber.

Finally, in Chapter 7 we apply the studies described above on photon+jet events collected during the run I. The use of data is of key importance to claim that our corrections are working fine. We will show how a $30 \%$ improvement in jet energy resolution, a major step towards better jet physics in Run II, is obtained. 


\section{Chapter 1}

\section{QCD and Jets in $p \bar{p}$ collision}

In this thesis we study the resolution with which the energy of "jets" produced in the collision of protons and antiprotons at the center of mass energy of $1800 \mathrm{GeV}$ is measured. This chapter provides a sketchy theoretical background of the process. Several concepts introduced in this chapter will be used in the following - mainly in chapter 5 where physics limitations to jet energy resolution will be treated.

\section{$1.1 \quad$ Introduction}

A "jet" is a group of particles which are produced in the collision of particles and are closely related to the directions of their momenta. According to the current theory (see section 1.2), each jet is a manifestation of a scattered sub-nuclear particle (parton). The partons make up all hadrons ${ }^{1}$ but cannot be separated as individual free particles - this property is called "confinement of hadrons" - due to the strong binding between them, that is supposed to become stronger if partons are moved away from each other. As a consequence of the stronger and stronger binding, an energetic parton that is trying to break away from the rest of the system loses its energy by pulling out from the vacuum more partons which then condense into a group of hadrons, that's a jet.

There are two classes of partons: the building blocks of matter, called quarks (spin one half), and the force-mediating particles (spin one), called gluons. A proton, for example, is built up of three quarks which are bound

\footnotetext{
${ }^{1}$ Neutron and proton turn out to be just the lightest particles in a spectrum of strongly interacting fermion state, called baryons, numbering near a hundred at the latest count. An equally numerous sequence of strongly interacting bosons, called mesons, has also been discovered, the pions being the lightest. All particles which undergo strong interactions, baryons and mesons, are collectively called "hadrons".
} 
together by "virtual" gluons (strong force). But when we look at it with a very powerful "microscope" - a very energetic particle beam - a different picture will appear, because the quarks are reabsorbing gluons and the gluons can in turn become quark pairs. Thus a hadron is seen by energetic probe as a cloud of quarks and gluons sharing the total hadron momentum. Furthermore the observed momentum distribution of partons in the nucleons is energy dependent - the wavelength used in the microscope - as more and more virtual emission is seen as we probe deeper. What happens when two hadrons collide it is sketched in fig. 1.1. A pair of partons may be one from each hadron according to some probability distribution, to make a hard collision. The scattered partons then make their ways into hadrons which will be detected by the detectors. This is picture is a refinement of the so called "parton model", since to understand experimental results a more sophisticated, and more global, theory of particle interactions is needed.

\subsection{QCD: the Standard Theory of Strong In- teractions}

A large amount of information about elementary particles and forces had been incorporated into an unified framework known as the "Standard Model" $[1,3]$.

According to the Standard Model, all matter is composed of two basic types of particles, quarks and leptons, and their corresponding antiparticles. The quarks and leptons come in several varieties ("families" or "flavors"), as listed in table 1.2. The standard model describes three forces (interactions) acting between these particles: the electromagnetic interaction between charged particles, described by Quantum Electrodynamics (QED) [2], the weak interaction unified with QED in the Glashow-Weinberg-Salam $S U(2) \times U(1)$ model [1] and the strong interaction between quarks and gluons, described by the $S U(3)$ gauge theory (Quantum Chromodynamics, QCD)[4].

QCD is a renormalizable theory similar to QED, in that quarks, which carry a strong charge called "color", interacts with gluons (analogous to photon in QED) via a Lagrangian similar to the QED Lagrangian. However, unlike QED, the gauge symmetry is non-Abelian, causing gluons also to posses color charge and consequently interact with themselves as well as with quarks. Unlike the QED case, the additional gluon-gluon interactions cause the strong coupling constant $\alpha_{s}$ to have a qualitatively different behaviour with $Q^{2}$ (the interaction momentum transfer scale) than the QED coupling 


\begin{tabular}{|l|c|c|c|c|}
\hline \hline QUARKS & \multicolumn{5}{|c|}{ flavor } & Charge \\
\hline & \multicolumn{5}{|c|}{} \\
\hline down-type quarks & $\mathrm{d}$ & $\mathrm{s}$ & $\mathrm{b}$ & $-\frac{1}{3}$ \\
\hline up-type quarks & $\mathrm{u}$ & $\mathrm{c}$ & $\mathrm{t}$ & $+\frac{2}{3}$ \\
\hline \hline LEPTONS & \multicolumn{5}{|c|}{ flavor } & Charge \\
\hline & $\mathrm{e}$ & $\mu$ & $\tau$ & -1 \\
\hline Charged leptons & $\nu_{e}$ & $\nu_{\mu}$ & $\nu_{\tau}$ & 0 \\
\hline Neutrinos & \multicolumn{5}{|c|}{} \\
\hline \hline
\end{tabular}

Table 1.1: Elementary point-like particles in the Standard Model.

constant $\alpha_{Q E D}$.

To a first approximation in $Q^{2} / \Lambda_{Q C D}$ one has:

$$
\alpha_{s}\left(Q^{2}\right)=\frac{4 \pi}{\left(11-\frac{1}{2} N_{f}\right) \ln \frac{Q^{2}}{\Lambda_{Q C D}}}
$$

where $N_{f}$ is the number of quark flavors with mass less than $Q$ and $\Lambda_{Q C D}$ is a parameter which, qualitatively, indicates magnitude of the scale at which $\alpha_{s}\left(Q^{2}\right)$ becomes strong. $\Lambda_{Q C D}$ is determinated experimentally to be about $0.2 \mathrm{GeV}$.

The constant $\alpha_{s}\left(Q^{2}\right)$ becomes large and perturbation theory breaks down at momentum transfer comparable with the masses of the light hadron, i.e. $Q^{2} \simeq 1 \mathrm{GeV}$. This could be an indication that the confinement of quarks and gluons inside hadrons is actually a consequence of the growth of the coupling at the low scales. This large value of the coupling constant is the source of most of the mathematical complexities and uncertainties that still surround QCD calculations at low $Q^{2}$. On the other hand it is of great importance that this "running" coupling goes to zero in the infinite $Q^{2}$ limit. This fact, called asymptotic freedom, allows perturbation theory to be used in theoretical calculations to produce experimentally verifiable predictions for hard scattering processes.

\subsection{QCD Improved Parton Model}

From the hadron picture above outlined, the proton and antiproton can be seen as "broad-band" beams of partons carrying varying fractions of the 
momentum of their parent hadron. One can picture this scattering process as a sequence of three different phases occurring at different time scales:

- partons approach each other with some momentum distribution - "Parton Distribution Function, PDF" - inside the parent hadron.

- A hard collision takes place between a pair of partons regarded as free particles. The cross section can be predicted by perturbation theory.

- New partons are generated by the two scattered partons and subsequently the quarks and gluons rearrange themselves into hadrons. This process is called hadronization or fragmentation.

In fig. 1.1 is sketched a $p \bar{p}$ interaction as pictured in the parton model.

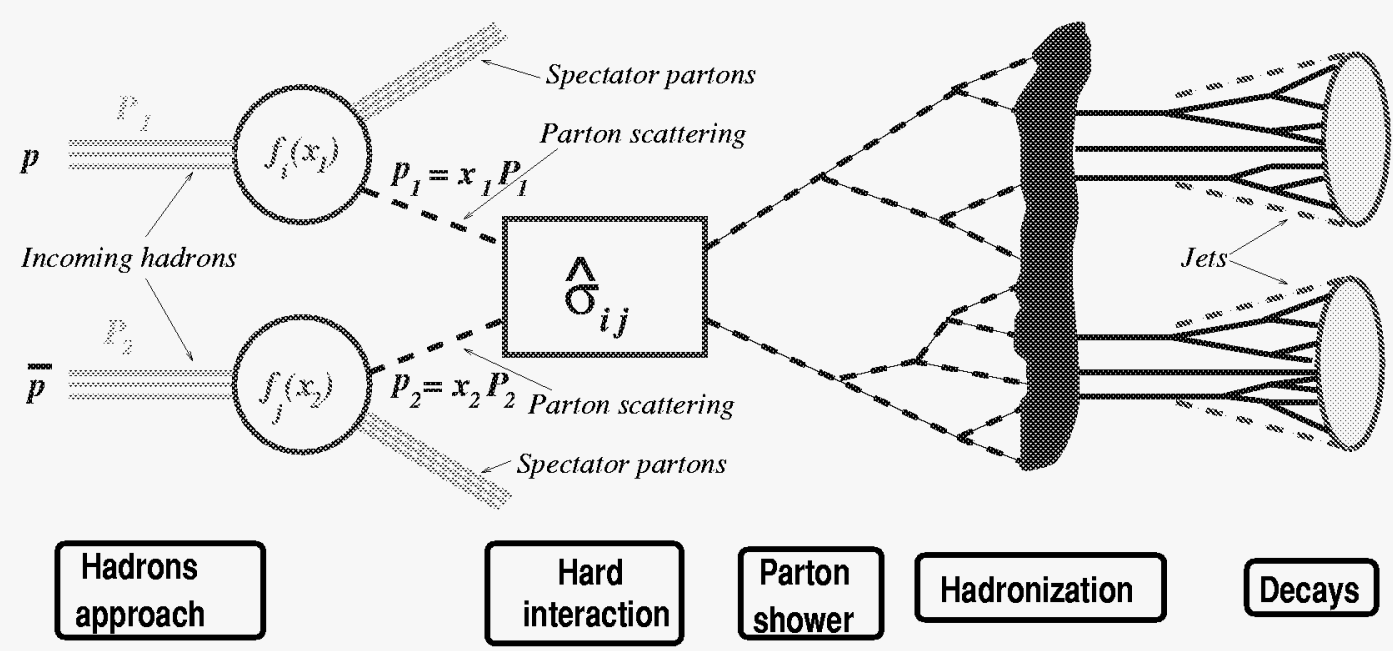

Figure 1.1: Sketch of a hard proton-antiproton interaction.

$P_{1}$ and $P_{2}$ are the momenta of the incident hadrons, with $p_{1}=x_{1} P_{1}$ and $p_{2}=x_{2} P_{2}$ being the momenta of the partons participating in the hard interaction. In the leading order the cross section for the scattering of parton of types $i$ and $j$ (denoted by $\hat{\sigma}_{i, j}$ ) is identical to the normal parton scattering cross section calculated in the same way as for a QED process.

The contributions from soft interactions which occur long before the hard scattering ${ }^{2}$ can be "factored out" and absorbed into the effective momentum

\footnotetext{
${ }^{2} \mathrm{~A}$ very important theoretical issue is whether the partons in hadron $H_{1}$, through the influence of their color fields, change the distribution of parton in hadron $\mathrm{H}_{2}$ before the hard scattering approximation is applied, thus spoiling the simple parton picture that we have outlined. Soft gluons which are emitted long before the collision are potentially trou-
} 
spectrum of the incoming hadrons, typically in the Parton Distribution Functions. In this way the remaining cross section involves only high momentum transfers (and therefore short times and distances) and in particular does not depend on the details of the hadron wave function or the type of hadrons. It is a single short-distance interaction and is computable in perturbation theory thanks to asymptotic freedom.

After the scattering, partons lose their energy in a perturbative evolution to a lower virtual mass $(\sim 1 \mathrm{GeV})$, thorough gluon bremsstrahlung and $q \bar{q}$ pair production.

Hadronization occurs at a much later time scale characterized by $1 / \Lambda$, where $\Lambda$ is the scale in $\alpha_{s}$ at which the coupling becomes strong. The interactions which change quarks and gluons into hadrons certainly modify the outgoing state, but they occur too late to modify the original probability for the scattering events to happen, which can therefore be calculated in perturbation theory.

Being jets the subject of this thesis, the fragmentation process will be discussed in much more detail, while only brief mention to Parton Distribution Functions and hard parton collisions will be given.

\section{Parton Distribution Functions (PDF)}

To compute the hard-process cross section we need to know the energy of the interacting partons. Usually one supposes that partons have a momentum fraction $x$ of the proton momentum ${ }^{3}$ with distributions as plotted in fig. 1.2. In the naive parton model, one defines distribution of parton $i, f_{i}\left(x, \mu^{2}\right)$, as the number of partons of kind $i$ within a high momentum proton with fractional momentum between $x$ and $x+d x$ ( $\mu$ is a factorization scale). These functions $f_{i}$ (one for each kind of partons) are what we have called parton distribution functions. They summarize the presumably intrigued interplay among partons in the proton projectile. In principle they are completely determinated by the QCD lagrangian, but bound states imply small $Q^{2}$ scales and therefore non-perturbative calculation. The only way to obtain these

blesome in this respect. This is a feature not present in process involving only one incoming hadron (as Deep Inelastic Scattering) but it is distinctive of hadron-hadron interactions. The theorem of factorization [5] states that since the initial and final distributions of partons interact on time scales that are vastly different from that of the hard scattering, the interference between the hard scattering and the initial and final states should be small. The property of factorization allows us to use the QCD parton model to describe the inelastic process.

${ }^{3}$ Transverse momenta of partons is neglected. 
functions is to extract them from experimental data. ${ }^{4}$

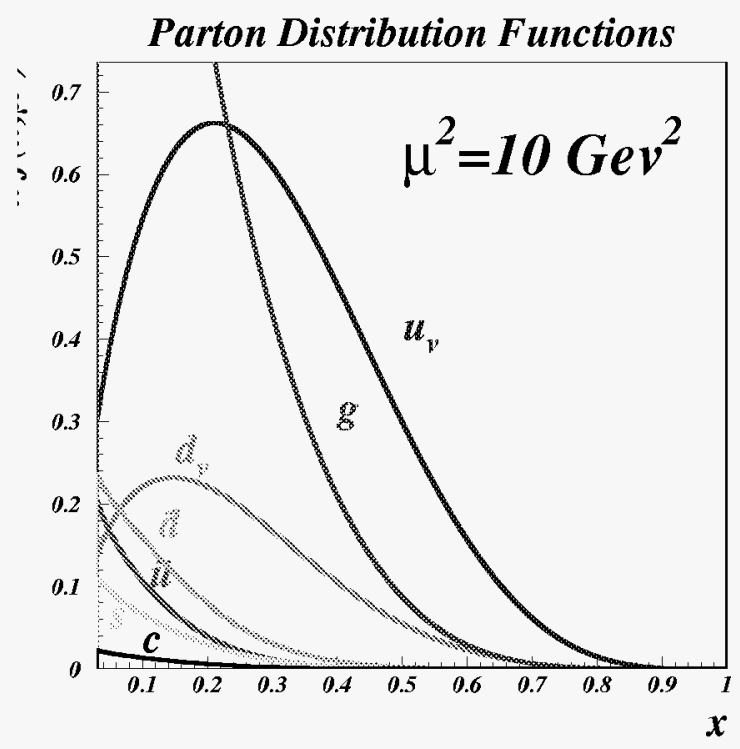

Figure 1.2: Parton distribution functions.

The factorization scale $\mu$ discriminates whether a parton, inside the incoming hadron, takes part in the hard scattering. In other words if the transverse momentum of a partons is greater than the scale $\mu$, it contributes to the short-distance cross section (as the partons $i$ and $j$ in the fig. 1.1). Instead, if its transverse momentum is less than the scale $\mu$, it is considered part of the hadron structure ("spectator partons").

\section{Hard Parton Collisions}

As described above, the scattering of two hadrons provides two broad-band beams of incoming partons. These incomings beams have a spectrum of longitudinal momenta determinated by the parton distribution functions. With the notations introduced above and in fig. 1.1, the cross section for a hard scattering process at CMS energy squared $s^{5}$ initiated by two hadrons with four-momenta $P_{1}$ and $P_{2}$ can be written as

\footnotetext{
${ }^{4}$ Of course data cover a finite range of $Q^{2}$. The evolution of the structure functions with $Q^{2}$, anyway, can be computed in perturbation theory, with the aid of the AltarelliParisi equation. The input to this equation are the structure functions measured at some energy, e.g. from Deep Inelastic scattering (DIS). A recent set of PDF [6] resulting for a global next-to-leading-order QCD fit to DIS and other data is shown in Fig. 1.2 at scale $\mu^{2}=10 \mathrm{GeV}^{2}$.

$$
{ }^{5} s=\left(P_{1}+P_{2}\right)^{2} \text {. }
$$
}




$$
\sigma\left(P_{1}, P_{2}\right)=\sum_{i, j} \int f_{i}\left(x_{1}, \mu^{2}\right) f_{j}\left(x_{2}, \mu^{2}\right) \hat{\sigma}_{i, j} \hat{s}, \alpha_{s}\left(Q^{2} / \mu^{2}\right) \delta\left(\hat{s}-x_{1} x_{2} s\right) d x_{1} d x_{2}
$$

The Feynman rules for calculating the cross section in terms of a perturbation expansion in the strong coupling $\alpha_{s}$ are determined from the QCD Lagrangian - complications from initial and final state $Q C D$ radiation ${ }^{6}$ are described later. The lowest order cross sections for elementary $2 \rightarrow 2$ parton process have been calculated by several authors [7]. They can be used to calculate the total cross section of any hard process $p \bar{p} \rightarrow X_{\text {spectators. }}$ In real events one or more extra partons can be produced within the hard scattering (" higher order process"), in which case we are dealing with parton subprocess of the form $2 \rightarrow 3,2 \rightarrow 4$, etc. However they are rare compared to the basic $2 \rightarrow 2$ interaction of partons. Moreover, the dividing line between initial and final state gluon radiation and hard higher order processes is experimentally ambiguous.

\subsection{Parton Shower and Jet Simulation}

Complete perturbative calculation in QCD have been performed only to nextto-leading order in most cases, or to one further order in $\alpha_{s}$ for a few observables. However, there are regions of phase space in which higher-order terms are enhanced and cannot be neglected. Indeed, whenever an external line of a QCD Feynman diagram with momentum $p$ and mass $m$, not necessarily small, emits a gluon of momentum $q$, there is a propagator factor in the amplitude of:

$$
\frac{1}{(p \pm q)^{2}-m^{2}}=\frac{ \pm 1}{2 p \cdot q}=\frac{ \pm 1}{2 \omega E(1-v \cos \theta)}
$$

where:

$\omega$ is the energy of the gluon,

E and $v$ the energy and velocity of the parton emitting it,

$\theta$ the gluon angle of emission.

Hence we can identify two special regions of phase space where these contributions diverge:

\footnotetext{
${ }^{6}$ In QCD the interacting quarks and gluons can radiate gluons in both the initial state, before the hard scattering, and in the final state, after the hard scattering.
} 
- for $v \cos \theta \rightarrow 1$ a gluon o light quark splits into two almost collinear partons having a "collinear enhancement";

- for $\omega \rightarrow 0$ a soft gluon is emitted at any velocity and emission angle.

As an alternative to a more and more precise prediction in perturbation theory, one may seek for an approximate result in which the leading contributions of these soft and collinear configurations can be identified and summed to all order, improving the convergence of the perturbation series. We shall see that this leads to a physically appealing "parton shower" picture which can be implemented in computer simulations, often called QCD MonteCarlo programs.

Formally the parton shower is first obtained with an approximate perturbative treatment of the QCD dynamics at scales of squared momentum transfer $t$ greater than some infra-red cut-offs value $t_{0}$, typically taken to be of the order of $1 \mathrm{GeV}^{2}$. The MonteCarlo method is particularly convenient because the perturbative treatment at $t>t_{0}$ can be combined with a nonperturbative model of the hadronization process, assumed to take place at scales $t<t_{0}$. In this way one obtains a QCD event generator, i.e. a program which provides a complete model for a given process involving the interaction and/or production of hadrons in a high energy $p \bar{p}$ interaction.

In the following section a heuristic description of the parton shower and a brief overview of the MonteCarlo method will be given. The discussion will highlight the physical meaning of the model rather than aim at a rigorous approach.

\subsubsection{Parton Branching}

In fig. 1.3 the collinear enhancement is associated with parton branching on a incoming or outcoming line of a QCD Feynman diagram. The shaded blobs represent the rest of the diagram. The branching line is shown for the gluon case.

There are two kinds of branching:

- spacelike branching: figure 1.3a) shows the kinematics for the case of branching on an incoming line. This branching will relate the process with incoming $b$ to that with incoming $a$ and emitted $c$.

- timelike branching: figure 1.3b) shows the kinematics for the branching of a parton $a$ into $b+c$.

Of course, in real events, several branching occur "in cascade". We consider next the fact of multiple branching, for example multiple gluon emission 

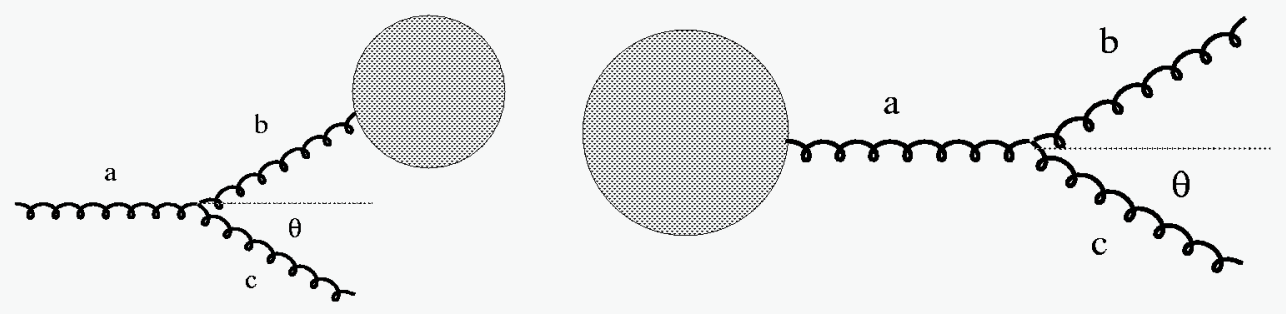

Figure 1.3: Parton branching of a) incoming parton (spacelike branching) and b) outgoing parton (timelike branching)

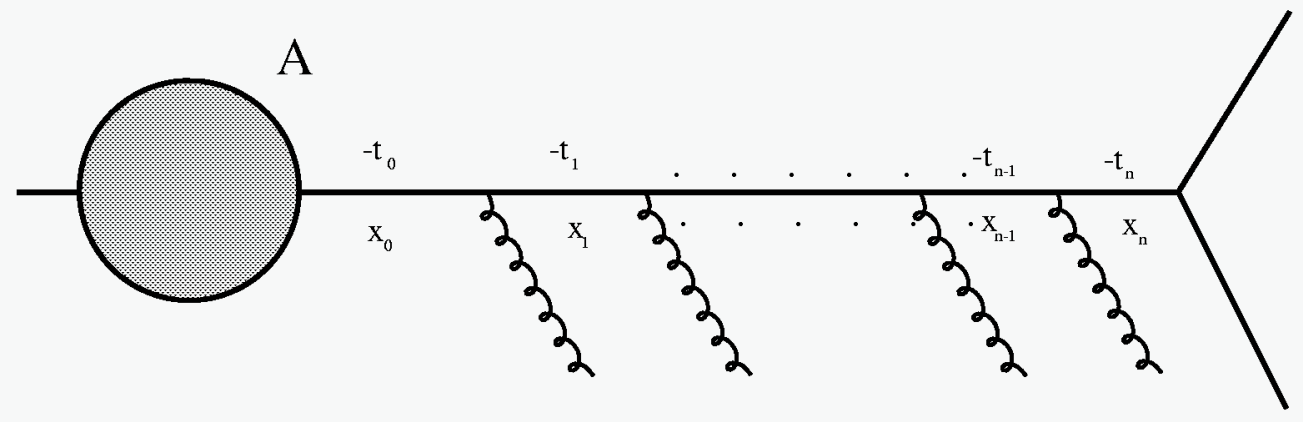

Figure 1.4: Initial state branching

from a spacelike quark, as illustrated in Fig1.4. An incoming quark from $A$, initially with a low virtual mass-squared $-t_{0}$ and carrying a fraction $x_{0}$ of the hadron momentum, moves to more virtual masses and lower momentum fractions by successive small-angle emissions. Eventually it participates in a hard scattering process at a scale $Q^{2}$.

The cross section for the hard scattering process will depend on the scale $Q^{2}$ and on the momentum fraction distribution of the parton seen at this scale, $f\left(x, Q^{2}\right)$. We can derive the evolution of the PDF $f(x, t)$ introducing a pictorial representation, which also is often used in the MonteCarlo simulation. We represent every sequence of branching by a path in $(t, x)$-space. One such path is shown in Fig 1.5. Each branching corresponds to a step downwards, from a higher to a lower value of the momentum fraction $x$, at a value of $t$ equal to (minus) the virtual mass-squared after the branching. At $t=t_{0}$, the paths have some distribution $f\left(x_{0}, t_{0}\right)$ characteristic of the hadron A at that scale. Following the path in the $(t, x)$ space and considering the change in parton distribution when $t$ is increased to $t+\delta t$ we can derive a differential equation - the Altarelli-Parisi equation - for $f(x, t)$ and find the structure function $f(x, t)$ of the parton evolved after all branchings, just before hard scattering. 7

\footnotetext{
${ }^{7}$ The above formulation is convenient for obtaining analytical solutions for the evolution of parton distributions. Often, to study more detailed features of the branching process,
} 


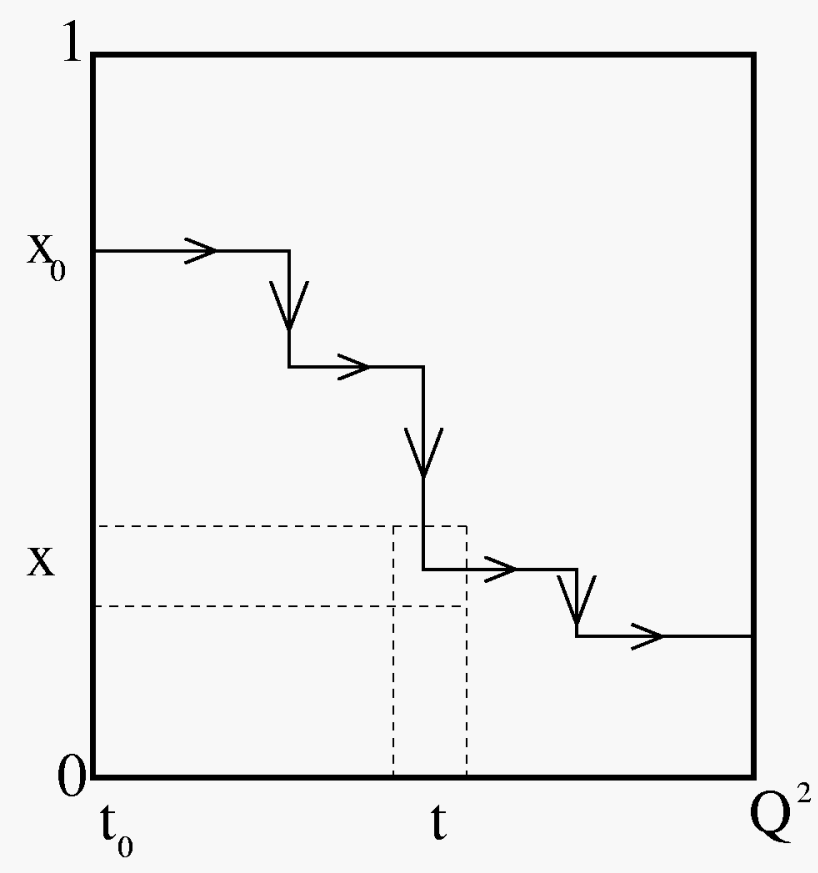

Figure 1.5: Representation of parton branching by path in $(t, x)$-space.

Each emitted gluon in Fig. 1.4, and in general each parton within a parton shower, can itself undergo further branching, which can be dealt by a similar algorithm. In the timelike branching of an outgoing parton, the same equation can be used to evolve $f(x, t)$, which now will represent the momentum fraction distribution of produced partons.

The main difference in the timelike case is that $t$ evolves downwards towards the cut-off value $t_{0}$ rather than upwards towards the hard process scale $Q^{2}$. As consequence of successive timelike branching, a parton cascade develops, as illustrated in Fig. 1.6. Each outgoing line becomes the source of a

and in particular to say anything about the structure of the corresponding final states, a numerical approach based on the MonteCarlo simulation technique is used.

First of all a function $\Delta(t)$ (called "Sudakov form factor"), that is the probability of evolution from $t_{0}$ to $t$ without branching, is introduced to integrate the Altarelli-Parisi equation. Hence, $\Delta(t) / \Delta\left(t^{\prime}\right)$ represents the probability of evolving from $t^{\prime}$ to $t$ without branching. The MonteCarlo program assumes a random branching rate

$$
\frac{\Delta(t)}{\Delta\left(t^{\prime}\right)}=\mathcal{R}
$$

where $\mathcal{R}$ is a random number distributed uniformly in the interval $[0,1]$ and performs a step-by-step evolution in the $(t, x)$ plane (see fig. 1.5). The value of $\left(t_{i}, x_{i}\right)$ generated by successive applications of the algorithm defines the virtual masses and momentum fractions of the exchanged quark, from which the momenta of the emitted gluons can be computed. 


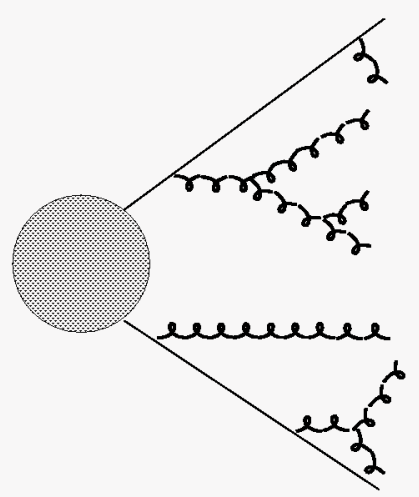

Figure 1.6: Parton shower in timelike evolution branching

new cascade, until a "no branching" step in the evolution of its virtual mass is reached. Those that do branch produce partons of lower virtual masses, which became more likely to generate "no branching" partons. Eventually all outgoing lines have stopped branching and the cascade process ends.

At this stage, which depends on the cut-off scale $t_{0}$, the outgoing partons have to be converted into hadrons via a hadronization model. Different available models are discussed in the next section.

\subsection{Hadronization Models}

After the parton shower has terminated, we are left with as set of partons with virtual mass-squared of the order of the cut-off scale $t_{0}$. From this point we enter the low momentum-transfer, long-distance regime in which non-perturbative effects become important. The main effect is hadronization which converts the partons into the observed hadrons.

One general approach to hadronization is the hypothesis of local partonhadron duality [8]. Here one supposes that the flow of the momentum and quantum numbers at the hadron level tends to follow the flow established at the parton level. Thus, for example, the flavor of the quark initiating a jet should be found in a hadron near the jet axis.

In order to make more detailed predictions, we need a specific hadronization model. Over the years, three classes of models have been developed, which will be outlined briefly in the following sections.

\subsubsection{Independent Fragmentation}

The simplest scheme for generating hadron distributions from those of partons is to suppose that partons fragment independently of each other. The 
original approach of Field and Feynman [9] was designed to reproduce the limited transverse momenta and approximate scaling of energy fraction distributions observed in quarks jets at moderate energy. The model assumes that quark jets can be analyzed on the basis of the following principles:

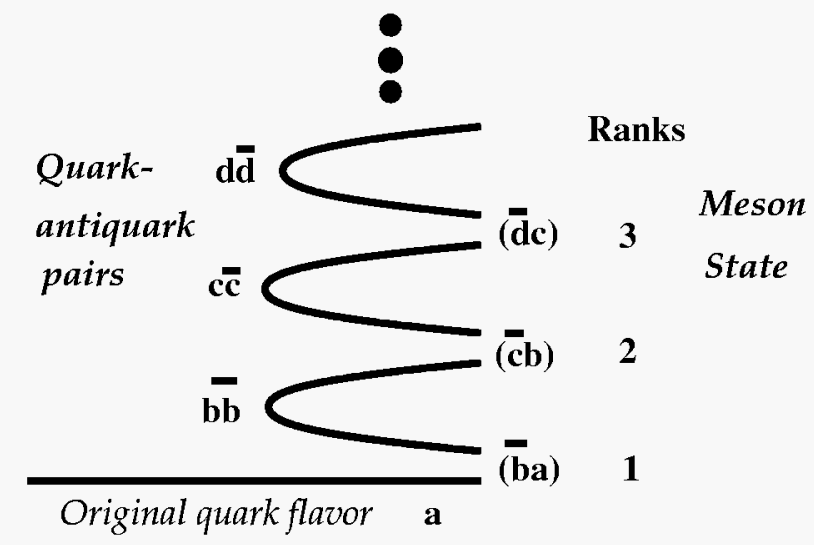

Figure 1.7: Hierarchy of mesons formed when an initial quark of flavor " $a$ " com-

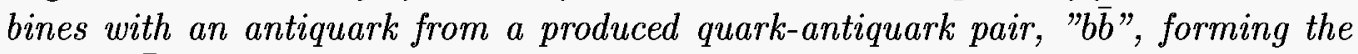
meson "ba" of rank 1. The remaining quark of flavor " $b$ " then combines with an antiquark from another produced quark-antiquark pair forming the meson of rank 2 and so on.

- A quark of flavor ' $a$ ' separating from the interaction region and having some momentum $P_{0}$ in the $\hat{z}$ direction creates a color field in which new $q \bar{q}$ pairs are produced.

- Quark ' $a$ ' then combines with an antiquark, say ' $\bar{b}$ ', from the new $b \bar{b}$ pair to form a meson of flavor $a \bar{b}$, leaving the remaining $b$ quark to combine with further antiquarks.

- A 'hierarchy' of meson is thus formed of which $a \bar{b}$ is first in 'rank', $b \bar{c}$ is second in rank, $c \bar{d}$ is third in rank, etc., as illustrated in Fig. 1.7.

- The 'chain decay' ansatz assumes that, if the rank 1 meson carries away the momentum $\xi$, from a quark of flavor ' $a$ ' and momentum $P_{0}$, the remaining cascade starts with a quark of flavor ' $b$ ' and momentum $P_{1}=P_{0}-\xi$. The remaining hadrons are distributed in precisely the same way as hadrons in a jet originated by a quark of flavor ' $b$ ' with momentum $P_{1}$.

- When the cascade stops after $n$ steps, the remain quarks $q_{n}$ that has not yet been assigned to hadron can no longer be treated independently. 
The unpaired quarks from all jets are together turned into hadrons ${ }^{8}$.

- The complete jet can eventually be described by the function $F(z)$ known as "fragmentation function" - defined as $F(z) d z=$ the probability of finding a meson independently of hierarchy with fractional momentum $z$ within $d z$ in a jet.

- For gluon fragmentation, the gluon is first split into a $q \bar{q}$ pair, either assigning all the gluon's momentum distribution to one or the other quark $(z=0$ or 1$)$ with equal probability, so that the gluon behaves as a quark of random flavor, or using the $g \rightarrow q \bar{q}$ Altarelli-Parisi spitting function. [10]

A weakness of the independent fragmentation scheme, as formulated above, is that the chains conserve momentum for each jet but not energy. Therefore it is necessary to rescale all momenta slightly after hadronization is completed to ensure the correct final energy.

\subsubsection{String Model}

When a color-neutral $q \bar{q}$ pair is produced, a color force field is created between them. It is believed that for a confining theory like QCD the color lines of force are mostly concentrated in a narrow tube connecting $q$ with $\bar{q}$, acting like a string with constant tension (independent of the separation between $q$ and $\bar{q})^{9}$.

The color force field, created by final partons, may generate a massless $q \bar{q}$ pair of zero energy-momentum at a point of the string. The string then splits into two independent color-neutral strings. As time develops the string breaks randomly into smaller pieces carrying smaller fraction of the original energy. When the invariant mass of a string piece gets small enough, it is identified as a hadron - or a cluster of hadrons - and the breaking stops within that piece. Thus the whole system eventually evolves into hadrons.

\footnotetext{
${ }^{8}$ If we are working in the lab frame these hadrons are slow and would play little part in determining the jet properties in an experiment.

${ }^{9}$ This picture is consistent with Regge phenomenology, heavy quarkonium spectroscopy and lattice QCD, which indicates a value of the string tension$$
\kappa=\frac{1 \mathrm{GeV}}{1 \mathrm{fm}} \approx 0.2 \mathrm{GeV}^{2}
$$ 
Since the breaking up of the string proceeds iteratively through $q \bar{q}$ pair creation. The string fragmentation approach does not look very different from independent fragmentation of the simple quark-antiquark system [11].

The string model becomes more distinct from independent fragmentation when gluons are present [12]. These are supposed to produce kinks on the strings. For example, in a color-neutral $q \bar{q} g$ system a single string runs from $q$ to $g$ to $\bar{q}$. So far, massless particles are present only to the end of the string. In this approach a massless gluon may point at the middle of the string.
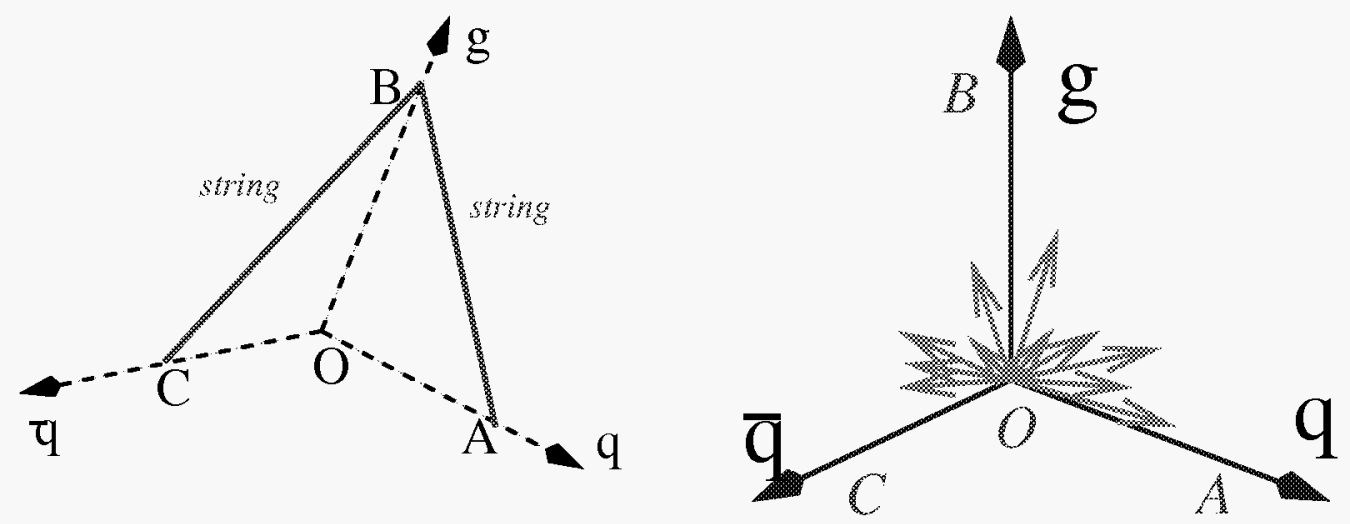

Figure 1.8: Working mechanism in the string model. a) A color-neutral $q \bar{q} g$ system connected by the string $A B C$ and b) its fragmentation. $A$ depletion of hadrons can be noticed in the COA region.

The fragmentation of the kinked string leads to an angular distribution of hadrons in three jet final states that is different from that predicted by independent fragmentation and in better agreement with experiment [13]. In a $q \bar{q} g$ system, there are only two string segments, $\mathrm{AB}$ and $\mathrm{BC}$ as illustrated in fig. 1.8a). The resulting hadron distributions are therefore not symmetrically distributed about the parton axes $\mathrm{OA}, \mathrm{OB}, \mathrm{OC}$ - which would be expected for independent jet fragmentation. Instead there is an excess of hadrons in the angular regions $\mathrm{AOB}$ and $\mathrm{BOC}$ with corresponding depletion in $\mathrm{COA}$. This has been called the string effect (fig. 1.8b)).

A schematic picture of the production of a multihadronic final state according to the string model is shown in Fig. 1.9. We note that, whenever a gluon splits perturbatively into a $q \bar{q}$ pair during the evolution of the parton shower, an additional string segment is produced. On the other hand, the gluons which remain at the end of the shower lead to kinks in the string segment which connects them. Each string segment then breaks up into hadrons as described above. 


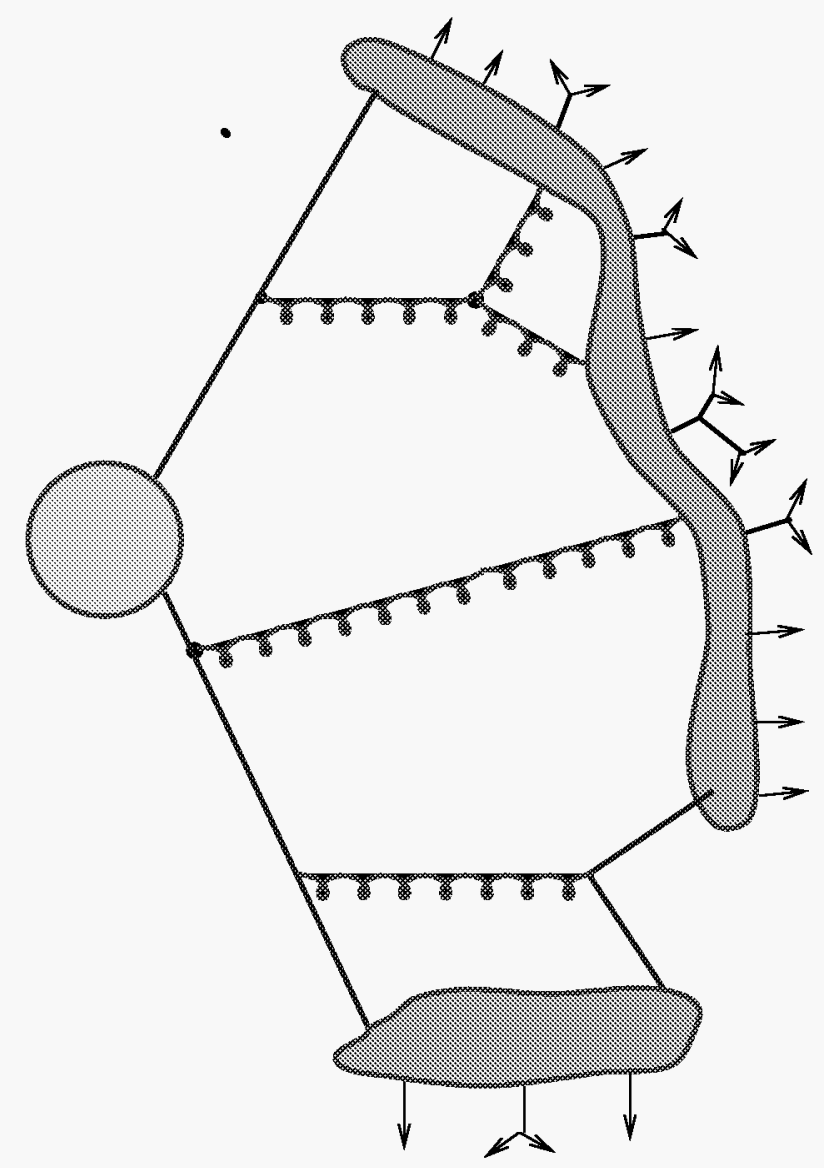

Figure 1.9: Parton shower in the string hadronization model.

\subsubsection{Cluster Model}

An important property of the parton branching process is the preconfinement of color [14]. In a class of cluster hadronization models, color-singlet clusters of partons form after the perturbative phase of jet development that then merge into the observed hadrons. The simplest way for color-singlet clusters to form after parton branching is through non-perturbative splitting of gluons into $q \bar{q}$ pairs [15]. Neighboring quarks and antiquarks can then combine into colored singlets. The resulting cluster mass spectrum is universal and steeply falling at large masses. Typical cluster masses are normally two or three times $\sqrt{t_{0}}$.

For normally adopted values of $t_{0}$, of order of $1 \mathrm{GeV}^{2}$ or less, most clusters have masses of up to a few $\mathrm{GeV} / \mathrm{c}^{2}$ and it is reasonable to treat them as superpositions of meson resonances. In a popular model [16], each cluster is assumed to decay isotropically in its rest frame into a pair of hadrons. One needs to invoke a more complicated decay scheme for the small fraction of 
clusters that have masses of more than a few $\mathrm{GeV} / \mathrm{c}^{2}$, for which isotropic two-body decay is an implausible hypothesis.

The hadronic energy and transverse momentum distributions predicted by this model agree quite well with experiment, without the introduction of any adjustable fragmentation functions. Also, the angular distribution in three-jet events is successfully described, as in the string model, provided soft gluon coherence is taken into account.

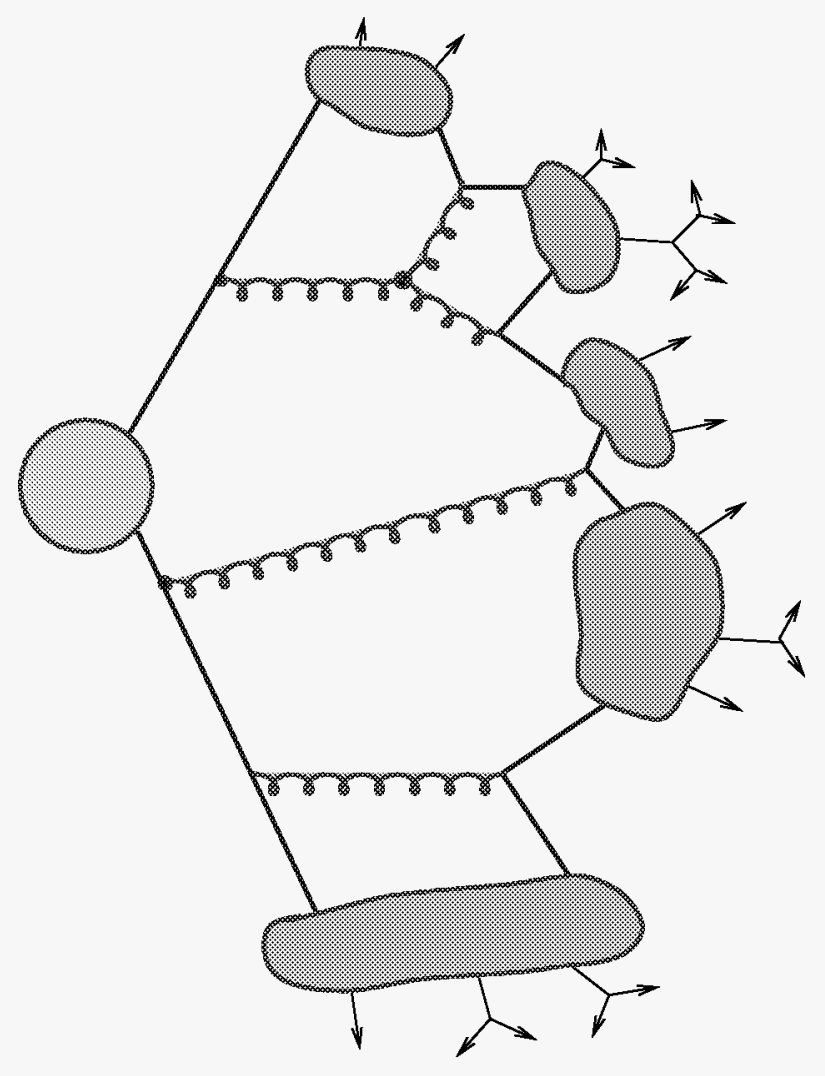

Figure 1.10: Parton shower in the string hadronization model, before final state clustering into hadrons.

Fig. 1.10 shows the cluster hadronization of the same parton shower as in Fig. 1.9. The gluons that remain at the end of the parton shower are split non-perturbatively into quark-antiquark pairs. The planar configuration of the shower determines that neighboring pairs (not from the same gluon) can form color-singlet mesonic clusters, which then undergo isotropic quasi-twobody decay into the observed partons. 


\subsection{QCD Events Generators}

There are several programs for generating full events from parton showers, using each one of the three above mentioned hadronization models. In this thesis two of those program will be used and compared (see chapter 5.2): the PYTHIA [17] and the HERWIG programs [18].

The string hadronization model outlined above, with many further refinements, is the basis of the JETSET simulation program [19] which, in combination with initial- and final-state parton branching, is used in the simulation program PYTHIA. The program HERWIG uses a low-mass cluster hadronization model [16] in conjunction with initial- final-state parton branching to simulate a wide variety of hard scattering process. 


\section{Chapter 2}

\section{Physics Motivations}

In this chapter the importance of jet energy resolution for Run I physics results and the role it will play in Run II will be described. After an introduction on CDF experimental program, we report top physics results (section 2.2), including analysis description and a discussion on uncertainty sources on the measured top mass. Section 2.3 describes the planned Top physics program in Run II. In section 2.4 a study on light Higgs search in Run II will be reported, showing the need for a better jet energy resolution if one wants to have solid discovery chance.

\subsection{Introduction}

In the year 2000 proton and antiproton will start again to collide inside the Tevatron ring after some years of shut-down. The machine will be upgraded and, thanks to the new Main Injector, luminosity will increase by about one order of magnitude up to $2 \times 10^{32} \mathrm{~cm}^{-2} \mathrm{~s}^{-1}$. At the same time the center of mass energy will increase from $1.8 \mathrm{TeV}$ to $2.0 \mathrm{TeV}$ and new physics will be on sight.

The goal of this new run is the accumulation of an integrated luminosity of $2 \mathrm{fb}^{-1}$ at $\sqrt{\mathrm{s}}=2.0 \mathrm{TeV}$ in the first two years of operation. After that the future of the Tevatron is not yet decided. Preliminary studies indicate that the average luminosity can be further increased, out that $20 \mathrm{fb}^{-1}$ of data can be accumulated before the new Large Hadron Collider at CERN will begin its run.

The increased luminosity requires extensive changes to experimental apparatus. Base on ten years of experience with CDF and Tevatron physics the detector is been upgraded with many powerful new features (for the details of CDF detector see chapter 3).

The new run moves the basic experimental program into a regime of 
precision physics. To exploit this chance it is fundamental to optimize the detector performance also in data acquisition and in offline analysis. Here again the Run I experience can play a decisive rule, because after 10 years we have learnt from the data acquisition performance limiting parameters and we can correct them for the new run.

From Run I data analysis, we have experienced that the main source of errors in many processes is the limited jet energy resolution. An improvement in jets resolution will have a big impact on the future Run II results.

In this chapter we will describe two important examples where an improved jets energy resolution will have a major impact: Top quark mass measurement and light Higgs Physics.

\subsection{Top Physics at CDF and Run I Experi- ence}

Tevatron Run I brought the discovery of the top quark, the first direct measurement of its mass and cross section [20, 21], and valuable first experience in top quark physics. In this section we will review the main results reached in semileptonic and all hadronic channel of top decay where jet physics had an important role, and the error in jet energy measurement caused the largest systematic error in the top mass. The new prospects opened by a better jet energy resolution in Run II will be discussed.

\subsubsection{Top Production and Decay Channels}

In a $p \bar{p}$ collision the top quark is expected to be produced in pairs, mostly by gluon-gluon fusion or by a quark-antiquark annihilation. Another weaker production mechanism would generate a single top by gluon-W fusion [22]. In the Standard Model framework the dominant top decay mechanism is $t \rightarrow W^{+} b\left(\bar{t} \rightarrow W^{-} \bar{b}\right)$. W is on-shell if $M_{t o p}>M_{W}$ as is the case. A $b$ quark generates a jet, while the $W$ can decay leptonically or hadronically. The independent decay of the top generates three final state topology. If at least one of the two $W$ s decays adronically, two topologies are generated that contain four or six jets in the final state as shown below.

- dileptonic channel: both $\mathrm{W}$ bosons decay leptonically (Fig. 2.1a) to a muon or electron. This is the channel with the smallest branching ratio $-5 \%$ adding the electron and the muon channel - but also the one with the best signal to background ratio. When the $W$ decays to a $\tau$ lepton, $W \rightarrow \tau \bar{\nu}_{\tau}$, the signal is confused by background. 

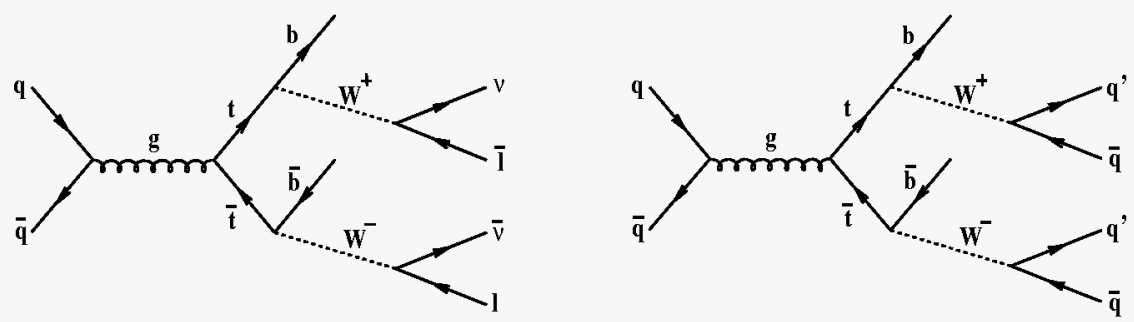

Figure 2.1: Top quark production: a) dilepton channel, b) all hadronic channel.
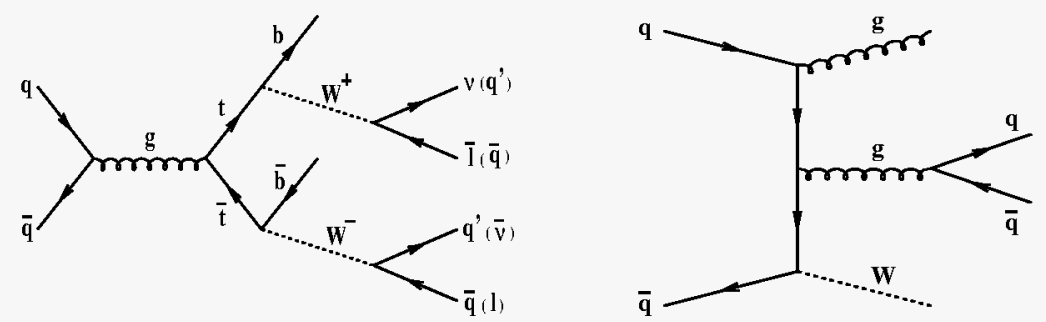

Figure 2.2: Top production: a) semileptonic channel, b) direct $W+$ multijets.

- semileptonic channel: one boson decays leptonically and the second decays hadronically (Fig. 2.2a). The final state presents one high $P_{T}$ lepton and some amount of $\mathbb{Z}_{\mathrm{T}}$ due to the neutrino. This channel has a large BR (30\%) and a fair signature. The main background comes from higher order process where $\mathrm{W}$ is produced directly together with jets ("W+multijets", Fig. 2.2b). The $W+$ multijets event rate is about 2-10 times higher than the $t \bar{t}$, depending on sample selection cuts. This sample will be sometimes referred as "lepton+jets".

- hadronic channel: both W bosons decay hadronically (Fig. 2.1b). This channel has the largest BR (44\%), but it is hard to disentangle it from the background because of the large QCD multijet cross-section.

\subsection{2 b-Tagging Algorithms}

All decay channels of the two top quarks have two $b$ quarks in the final state, that, after fragmentation, generate typically $B^{ \pm}$mesons. It was fundamental for the top quark discovery to recognize jets whose parent parton was a b quark - an event where one jet is recognized as a b-jet is called " $b$-tagged" event. Therefore, a "b-tagged" event is a top candidate event" ${ }^{1}$. Moreover, this kind of jets have some interesting properties and different corrections have to be applied with respect to other jets.

\footnotetext{
${ }^{1}$ The ratio signal/background falls off from 0.5 for pre-tagged events to 4 for b-tagged events.
} 
It is possible to recognize b-jets thanks to long decay length of a meson in the jet - a meson $B^{ \pm}$has a mean life $\tau_{o} \approx 10^{-12} \mathrm{~s}$ with a decay length $c \tau_{o} \approx 462 \mu \mathrm{m}$. The Silicon Vertex Detector (SVX) can reconstruct the tracks of charged particles with a resolution of about $13 \mu \mathrm{m}$ in the $r-\phi$ plane, more than adequate to signal $B$ decays. Two b-tagging algorithms are used in the CDF data analysis: SVX and SLT tag.

\section{SVX, the Jet-Vertexing Algorithm}

The SVX $b$-tagging algorithm looks for secondary vertices in jets due to $b$ decay. The first step is the seed vertex search. A candidate seed for the secondary vertex is searched from two good tracks found in the SVX detector falling inside a cone around the jet - cuts to remove photon conversions, $K_{s}$ and $\Lambda$ decay tracks are applied. Then, all SVX tracks falling inside the cone and passing a cut on absolute impact parameter are associated to the seed and a constrained fit is made. As a product of such a fit, the length between primary and secondary vertex is calculated. Finally if the number and quality of tracks associated to the secondary vertex pass some final requirements, the jet is said "SVX tagged".

Accurate Monte Carlo simulations of $t \bar{t}$ events passing top candidate sample selection cuts have shown that SVX tags $t \bar{t}$ events in the single lepton channel have an efficiency of $(42 \pm 5) \%$.

\section{SLT, the Soft Lepton Finder Algorithm}

The second technique for tagging $b$ quarks (SLT tagging) consists in looking for an additional lepton from semileptonic $b$ decay. Electrons and muons are found by matching CTC tracks with electromagnetic energy clusters or stubs in the muon chambers. To maintain acceptance for leptons coming directly from $b$ decay as well as from the daughter $c$ quark, the $P_{T}$ threshold is kept low $(2 \mathrm{GeV} / \mathrm{c})$. The major backgrounds in the SLT analysis are hadrons that are misidentified as leptons, and electrons from unidentified photon conversions.

The overall tagging efficiency for $t \bar{t}$ single lepton candidate events passing the mass sample selection cuts is computed with Monte Carlo simulation to be $(20 \pm 4) \%$. The fake background rate is estimated in $(4 \pm 1) \%$.

\subsubsection{Semileptonic Channel}

The top quark mass presented in the CDF "evidence" [23] and "observation" [24] publications was obtained from a 2-constraint fit to samples of single lepton +4 jets candidate events. At present, top mass measurement is 
being made also in the 6 jets and in the dilepton samples but the best value (see fig. 2.5 ) is obtained in the single lepton channel. More information can be found in $[25]$.

\section{Data Sample}

The data sample used to measure the top quark mass is a subsample of the one used to measure the $t \bar{t}$ production cross section ${ }^{2}$. To select the sample, the following cuts are applied to the inclusive single lepton sample to reduce the background:

- one isolated $e$ or $\mu$ of large $E_{T}$ :

$-E_{T}>20 \mathrm{GeV}$

$-|\eta|<1.1^{3}$

- The lepton is required to be isolated $\left(I_{\text {cal }}^{4}<0.1\right.$ and $\left.I_{t r k}{ }^{5}<0.1\right)$.

- Large missing $E_{T}$ : $\mathbb{Z}_{\mathrm{T}}>20 \mathrm{GeV}$.

- At least 3 large $E_{T}$ jets: $E_{T}>15 \mathrm{GeV}$.

In addition, the following clean-up filters are applied:

- all events with dilepton topology are rejected;

- no isolated tracks other than the one of the high $P_{T}$ lepton is allowed;

- the primary vertex $z$ coordinate should be small because of the limited SVX acceptance: $\left|z_{\text {vertex }}\right|<60 \mathrm{~cm}$.

In the sub-sample used for reconstructing the top quark mass, a fourth jet was also required. For the fourth jet, a loose cut was applied: $E_{T} \geq 8 \mathrm{GeV}$ and $|\eta| \leq 2.4$. This 4-jets sample is usually referred as "pretag lepton+jets" sample. The "tagged lepton+jets" mass sample is selected by requiring at least one jet to be tagged by at least one of the $b$-tagging algorithms (SVX or SLT).

\footnotetext{
${ }^{2}$ The integrated luminosity of the sample is $109 \mathrm{pb}^{-1}$

${ }^{3} \eta$ is the pseudorapidity defined as $\eta=\ln \left(\operatorname{tg} \frac{\theta}{2}\right)$, where $\theta$ is the polar angle relative to the proton beam.

${ }^{4} I_{\text {cal }} \times E_{T}^{\text {lepton }}$ is the transverse energy in the towers within a cone of radius $R=0.4$ centered on the electron but excluding the electron cluster's transverse energy.

${ }^{5} I_{t r k} \times P_{T}^{\text {lepton }}$ is the $P_{T}$ sum of CTC tracks within a cone $R=0.25$, excluding the lepton track contribution.
} 


\begin{tabular}{cc}
\hline \hline Subsample & Measured $M_{\text {top }}\left(\mathrm{GeV} / \mathrm{c}^{2}\right)$ \\
\hline$S V X$ double tag & $170.1 \pm 9.3$ \\
$S V X$ single tag & $178.0 \pm 7.9$ \\
$S L T$ tag $($ no $S V X)$ & $142_{-14}^{+23}$ \\
No tag $\left(E_{T}\left(j_{4}\right) \geq\right.$ & $180 \pm 9.0$ \\
\hline \hline
\end{tabular}

Table 2.1: Subsamples of $W+\geq 4$ jet events which are used for the top quark mass measurement. For each subsample the measured top mass is shown. The quoted uncertainties are statistical only.

\section{Mass Reconstruction}

We summarize briefly the procedure to extrapolate the top mass from the selected data sample:

- For each selected event, the 4 leading (in $E_{T}$ ) jets are associated to the 4 partons in a $t \bar{t}$ semileptonic decay: $b, \bar{b}, q$ and $\bar{q}^{\prime}$.

- A "kinematic $\chi^{2}$ " is built with $\mathrm{M}_{\mathrm{TOP}}$ as free parameter and where constraints kinematic is applied.

- The same method is repeated and a $\chi^{2}$ is found for any combination of the associations among jets and partons

- The lowest $\chi^{2}$ decides which is the correct combination, and the relative $M_{t o p}$ is the top mass for that event.

- The distribution of these values is analized in order to derive a top mass quark indicated by the sample as a whole. The top contribution is computed as a function of the top mass and suitably parametrized. The background shape is also parametrized by Monte Carlo and a likelihood function $\mathcal{L}$ is made for the observed distribution to be obtained as a weighted sum of a (top-mass dependent) signal distribution plus the background distribution.

- The likelihood $\mathcal{L}$ is maximized with respect to all parameters introduced and $M_{\text {top }}$.

- An analysis optimization can be performed dividing the sample in four subsamples with similar b-tag characteristics. The final result is the average of the results for the four independent samples. Table 2.1 summarizes the fit results for each subsample. 
Figure 2.3 is the reconstructed mass distribution of the average of the four subsamples defined above, together with the result of the combined fit. The inset shows the negative log-likelihood as a function of top mass, from which we can derive the statistical error ${ }^{6}$.

The fit result gives as best value for the top quark mass (statistical error only)

$$
M_{\text {top }}=175.8 \pm 4.8 \mathrm{GeV} / \mathrm{c}^{2}
$$

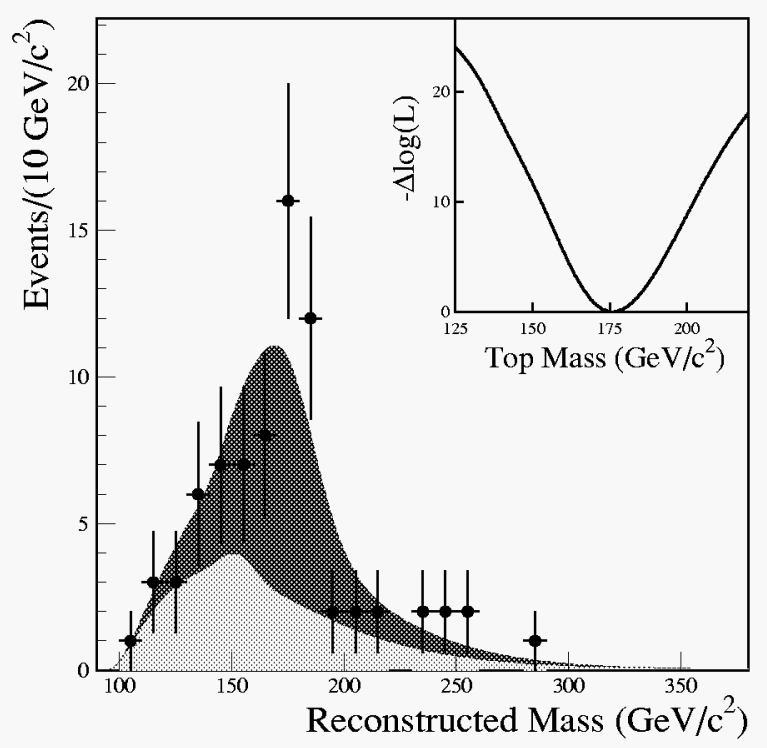

Figure 2.3: Reconstructed mass distribution of the combined four subsamples. The data (points with error bars) is compared with result of the combined fit (top + background, dark shading), and with the background component only (light shading). The inset shows the variation of the combined negative log-likelihood with top mass.

\section{Systematic Uncertainties}

The top quark mass measurement is subject to several kinds of systematic uncertainties some of which are due to the sophisticated method used to compute the mass. For some source of uncertainty is not only difficult to estimate the error, for others it is even a problem to define it. Here we would like only to summarize the sources of uncertainty and their physical origin and meaning. More details will be found in references.

\footnotetext{
${ }^{6}$ Assuming gaussian errors, the error on the mass is taken as the mass difference at which the negative log-likelihood increases by $1 / 2$.
} 
- Jet $E_{T}$ scale and soft gluon radiation: the largest systematic comes from the jet energy measurement. Chapter 3 deals with this subject. The final systematic uncertainty is $\pm 4.4 \mathrm{GeV} / \mathrm{c}^{2}$.

- Radiation Effect: this uncertainty is due to high transverse momentum gluons which are radiated from the initial or final state of a $t \bar{t}$ event and sometimes take the place of $t \bar{t}$ decay products among the four leading jets (parton-jet misassociation) ${ }^{7}$. The amount of systematics from initial state radiation is estimated in $1.4 \mathrm{GeV} / \mathrm{c}^{2}$. For the final state radiation is $1.1 \mathrm{GeV} / \mathrm{c}^{2}$.

- Shape of the background spectrum: the background shape is an important parameter in the likelihood fit. The impact of the uncertainty of his shape was monitored by assuming a number of different shapes. The systematics associated to this uncertainty is $1.3 \mathrm{GeV} / \mathrm{c}^{2}$.

- b-tagging bias: b-tagging can generate a bias because of the uncertainty in efficiency versus $E_{T}$ and in the rate of tagging non-b jets in real top events. The amount of systematic for this effect is $0.4 \mathrm{GeV} / \mathrm{c}^{2}$.

- Parton distribution function: Uncertainty in these functions induces an error of $0.3 \mathrm{GeV} / \mathrm{c}^{2}$.

- Monte Carlo generator: the difference between result obtained using different Monte Carlo models indicates an uncertainty of $0.1 \mathrm{GeV} / \mathrm{c}^{2}$.

Table 2.2.3 summarizes all sources of uncertainties.

\subsubsection{All Hadronic Channel}

In this channel systematic errors are dominated by jet energy resolution and hard gluon effects. It is important to emphasize how a better jet resolution can reduce the top mass uncertainty in this channel.

\section{Data Sample}

The kinematical selections are:

- $N_{j e t} \geq 6\left(\right.$ and $\left.N_{j e t} \leq 8\right)$

\footnotetext{
${ }^{7}$ The final state radiation impacts the top mass in two different ways. The amount of final state radiation affects the energy distribution within jets and adds additional jets to events. Formally, the first one is already accounted for in the jet energy scale systematics. So, for "final state radiation" we mean only the second effect.
} 


\begin{tabular}{|c|c|}
\hline \hline Source & Value $\mathrm{GeV} / \mathrm{c}^{2}$ \\
\hline Jet energy scale & 4.4 \\
Initial state radiation & 1.4 \\
Final state radiation & 1.1 \\
Shape of background spectrum & 1.3 \\
b-tag bias & 0.4 \\
Parton distribution function & 0.3 \\
Monte Carlo generator & 0.1 \\
\hline Total & 4.9 \\
\hline \hline
\end{tabular}

Table 2.2: Systematic uncertainties on the top mass measurement in the semileptonic channel.

- $\Delta R_{\min } \geq 0.5^{8}$

- $\sum E_{T} \geq 200 \mathrm{GeV}$

- $\sum E_{T} / \sqrt{\hat{s}} \geq 0.75$

- Aplanarity ${ }^{9}+0.0025 \times \sum_{3}^{N} E_{T} \geq 0.54$

\section{Reconstructed Mass}

The fit for top mass is made on a subsample with a supplementary requirement of one or more b-tags. Figure 2.4 shows the mass distribution and the $\log$-likelihood distribution with the similar meaning as for to semileptonic channel.

The best fit mass is:

$$
M_{t o p}=187 \pm 8 G e V / c^{2}
$$

\section{Systematic Uncertainties}

The systematic error is summarized in table 2.2.4. The meaning of each entries has been previously discussed.

\footnotetext{
${ }^{8} R_{\min }$ is the distance between the two closest jets in the $\eta-\phi$ space.

${ }^{9}$ The Aplanarity of an event lies in the range $0 \leq A \leq \frac{1}{2}$. A is small for coplaner events and big for roughly "spherical" events.
} 


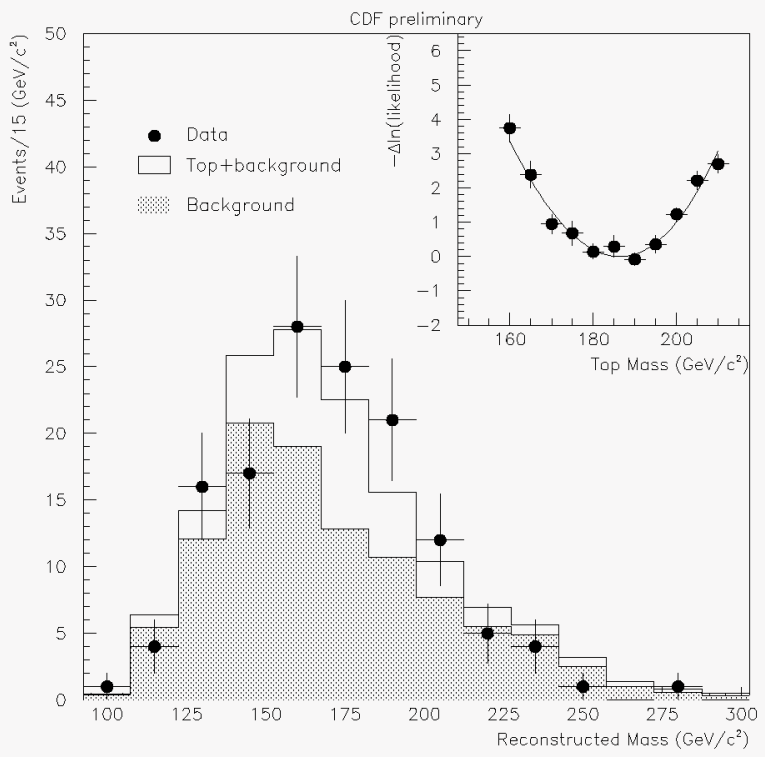

Figure 2.4: Top mass reconstruction in all hadronic channel. The observed events are 136 with at least one b-tag. The data (points with error bars) is compared with result of the combined fit (top + background, full line), and with the background component only (light shading). The inset shows the variation of the combined negative log-likelihood with top mass.

\begin{tabular}{|c|c|}
\hline \hline Source & Value $\mathrm{GeV} / \mathrm{c}^{2}$ \\
\hline Jet energy scale & 6.21 \\
Hard gluon radiation & 8.0 \\
Shape of background spectrum & 1.71 \\
b-tag bias & 0.17 \\
Parton distribution function & 5.02 \\
Monte Carlo generator & 0.35 \\
\hline Total & 12 \\
\hline \hline
\end{tabular}

Table 2.3: Systematic uncertainties on the top mass measurements in all hadronic channel.

\subsection{Top Quark Mass in Run II}

The Top quark mass will be one of the most important electroweak measurements to be performed at the Tevatron. In combination with the $W$ mass, $m_{t}$ gives information about the mass of the standard model Higgs boson.

Figure 2.5 shows the top mass resolution achieved in Run I and figure 2.6 


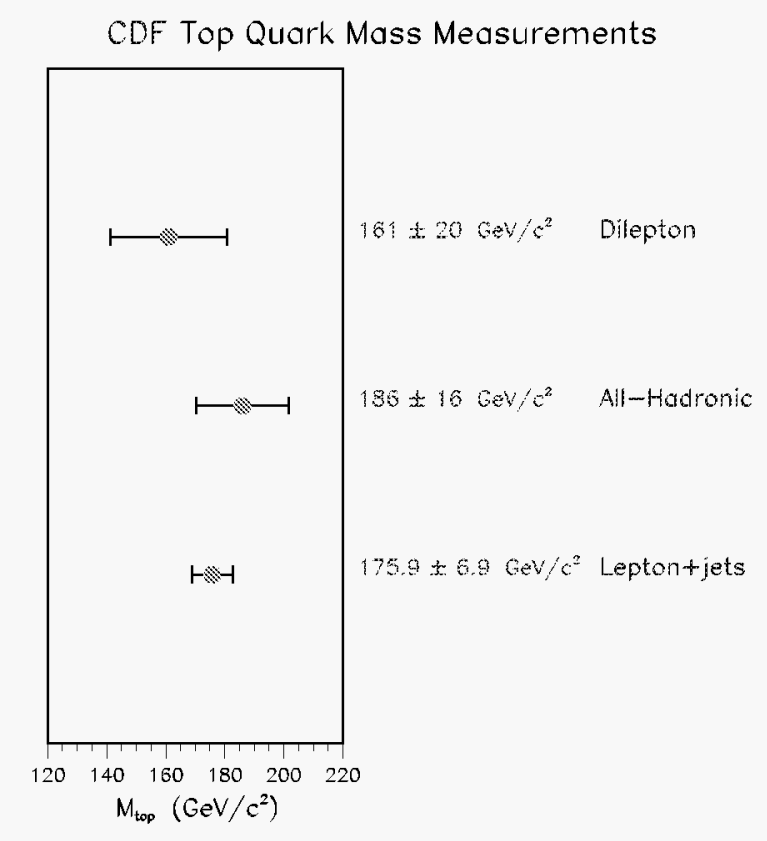

Figure 2.5: The CDF top mass values in each decay channel: statistical and systematic uncertainties are folded in quadrature.

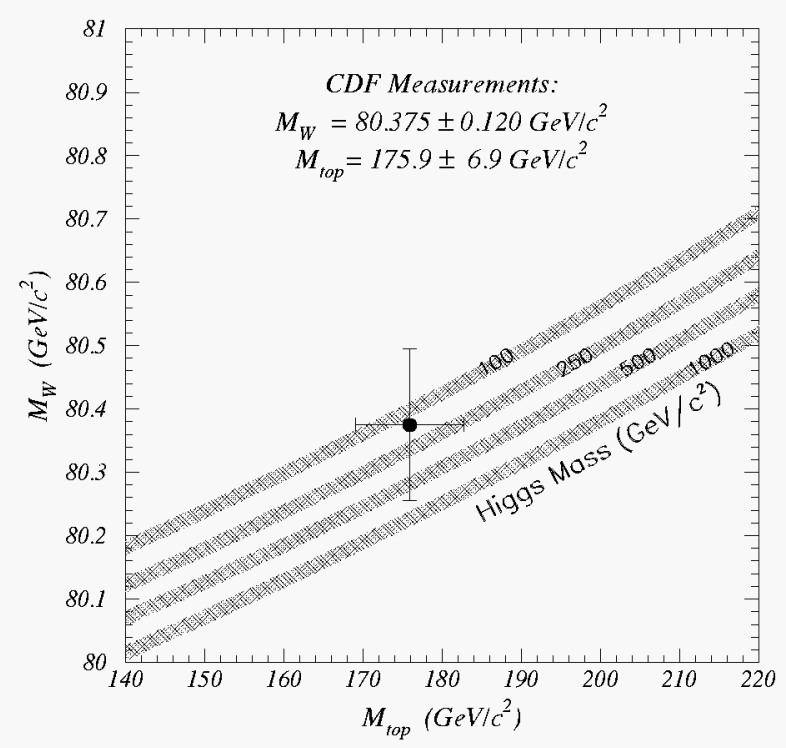

Figure 2.6: $C D F$ measurement of $M_{W}$ and $M_{\text {top }}$ and Higgs mass prediction. The curves are from a calculation [26] of the dependence of $M_{W}$ and $M_{\text {top }}$ in the minimal Standard Model using several Higgs masses. The bands are the uncertainties obtained by folding in quadrature the uncertainties on $\alpha\left(M_{Z}^{2}\right), M_{Z}$, and $\alpha_{s}\left(M_{Z}^{2}\right)$ 
shows the predicted top and $W$ mass measurements constraint on the Higgs mass with the present resolution on the top mass.

Currently, the statistical and systematic uncertainties on CDF top mass measurement are both about $5 \mathrm{GeV}$. The statistical uncertainty should scale as $1 / \sqrt{N}$. The CDF collaboration is confident to reduce the statistical uncertainty - in the optimized lepton $+\geq 4$-jet sample with at least one b-tagged jet - below $1 \mathrm{GeV} / \mathrm{c}^{2}$.

In Run II the uncertainty on the top mass will be dominated by systematics. Because of new integrated tracking, the acceptance for doubletagged lepton $+\geq 4$-jet events will increase by about a factor of 2.5 . In these double-tagged events, the probability of misassociation among jet and parton is lower, reducing this kind of systematic uncertainty. Moreover the b-tagged bias may be better understood for this class of events.

Almost all of the systematic uncertainties in the top mass measurement are coupled to the reliability of the Monte Carlo models to get the spectrum of fit masses in signal and background. Assuming that the theory model is accurate, most of the uncertainty on the jet energy is related to resolution effects. Instrumental contribution include calorimeter nonlinearity, losses in cracks, dead zones and absolute energy scale. A larger and more difficult part of the energy resolution concerns the reliability of the extrapolation to the original parton energy. Once again, a jet resolution study appears of great importance to improve the quality of physics results reachable in Run II.

\subsection{Light Higgs Physics in Run II}

One of the primary goals of present and future colliders is to understand the mechanism responsible for the spontaneous breaking of the $S U(2)_{L} \times U(1)_{Y}$ electroweak symmetry [27]. The simplest model for this mechanism is the standard Higgs model, based on a doublet of fundamental scalar fields. This model predicts the existence of a new particle, the Higgs boson, of unknown mass, but with fixed couplings to other particles. The search for the Higgs boson represents a benchmark in our search for the mechanism of electroweak symmetry breaking.

The current lower bound on the Higgs mass is $64.5 \mathrm{GeV}$ from LEP. In the near future the increased LEP energy will allow to cover masses up to $m_{H} \approx 95 \mathrm{GeV}$.

Much higher masses will be explored by the CERN Large Hadron Collider (LHC), which will cover from $m_{H} \approx 130 \div 170 \mathrm{GeV}$ at full energy and luminosity [28, 29].

However, the light intermediate-mass region, $m_{H} \approx 80 \div 130 \mathrm{GeV}$, which 
is also the favored region for a light Higgs boson predicted in the minimal supersymmetric theory, is the most difficult to investigate at the LHC. The CMS detector intends to cover this region with the rare decay $H \rightarrow \gamma \gamma$ [28]. Also the ATLAS detector covers down to $m_{H} \approx 110 \mathrm{GeV}$ with this mode, and would require $500 \mathrm{fb}^{-1}$ to cover down to $m_{H} \approx 80$ [29].

The dominant decay mode of the Higgs boson in this mass range is $H \rightarrow b \bar{b}$ with the a branching ratio of about $80 \%$. It is been suggested that the process $q \bar{q} \rightarrow W H$, followed by $H \rightarrow b \bar{b}$ and the leptonic decay of the $W$ boson, could be used at the Tevatron to discover the light intermediate-mass Higgs boson $[30,31,32]$. This signal may be more difficult to detect at LHC due to very large top-quark background.

It is vital for CDF that we do not leave the intermediate-mass Higgs window open, and the Run II can potentially play a crucial role in closing this window. In the next paragraph the conditions to discover a light Higgs boson will be described.

\subsection{1 $q \bar{q} \rightarrow W H, H \rightarrow b \bar{b}$}

The associate production of a Higgs boson and a $\mathrm{W}$ or $\mathrm{Z}$ boson, with the Higgs decaying to $b \bar{b}$ and the $W$ or $Z$ decaying leptonically $(e$ or $\mu$ ), is a possible way to detect the Higgs in the mass range $60 \div 130 \mathrm{GeV}$.

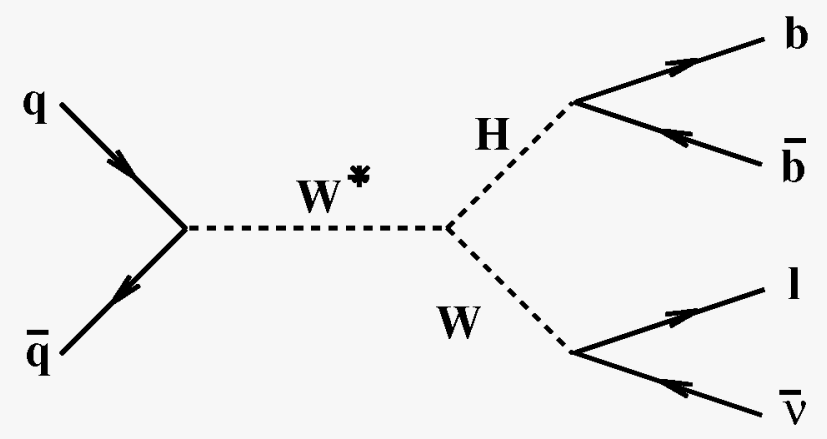

Figure 2.7: Production of Higgs boson in the process WH with $H \rightarrow b \bar{b}$

The main source of background simulating the Higgs decay to two jets will be $W+2$ jet events ${ }^{10}$.

\footnotetext{
${ }^{10}$ Other worthy backgrounds to WH process containing two heavy quarks each are:

1) the $W b \bar{b}$ process;

2) the WZ with $Z \rightarrow b \bar{b}, t \bar{t}$;

3) $W^{*} \rightarrow t b$;

4) W-gluon fusion $(t+q+b$ final state);

5) $W c \bar{c}$.
} 
From Run I data we would expect in Run II $W+2$ jets backgrounds to be 1000 times larger than signal. But thanks to $b$ tagging we can reduce by a great deal this background. Moreover the upgrades of CDF detector ${ }^{11}$ are expected to improve both fiducial acceptance to $|\eta| \lesssim 2.0$ and efficiency of b-tag algorithm.
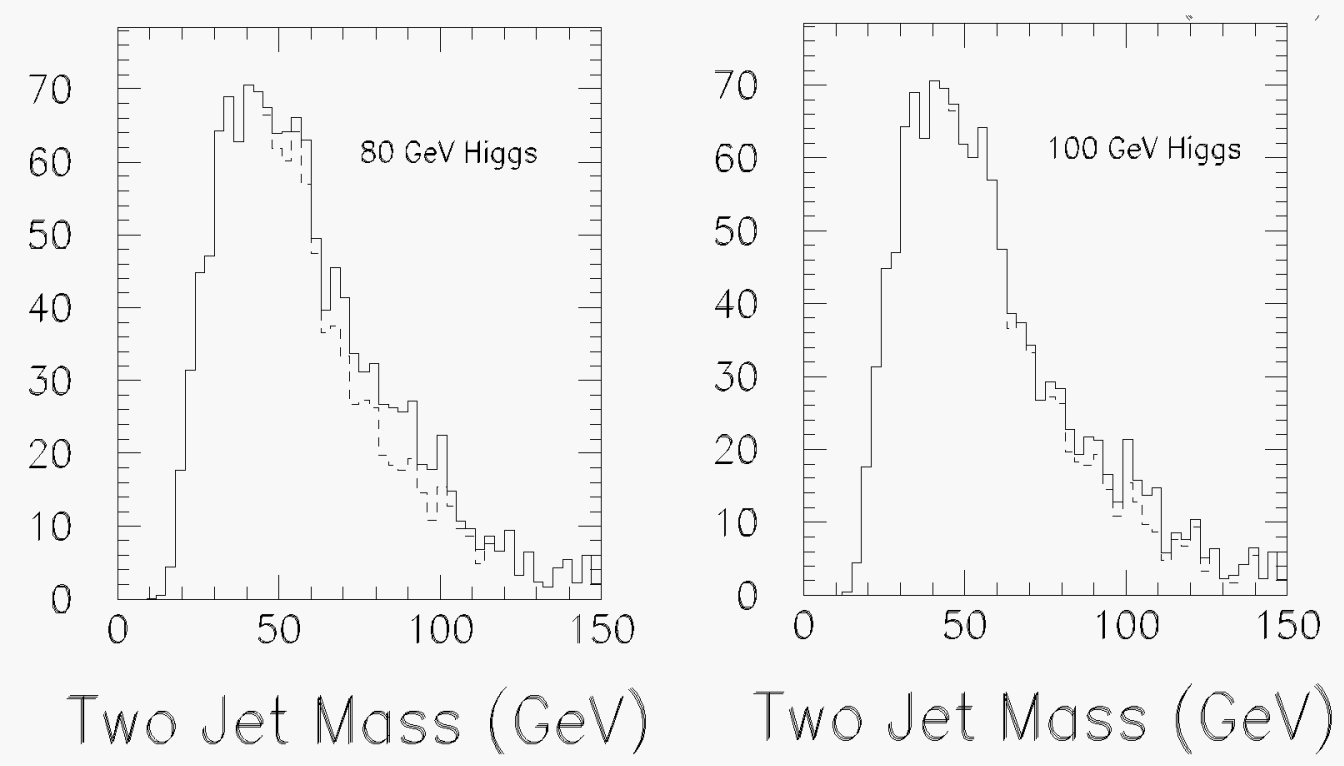

Figure 2.8: Expected signal+background mass distribution for the WH process with $10 \mathrm{fb}^{-1}$ of data at $2 \mathrm{TeV}$. The solid line is signal+background, the dashed line the sum of all backgrounds. The nominal jet resolution is assumed.

In figure 2.8 we can see the expected signal+background distributions using $10 \mathrm{fb}^{-1}$ of data for Higgs masses of 80 and $100 \mathrm{GeV}$. The present jet resolution is used in this plot. Clearly this resolution is too bad to see anything.

Figure 2.9 is the same plot but a better (by about $30 \%$ ) jet resolution is assumed, as we expect can be achieved with more study. Now the signal begins to emerge. The issue of a better jet energy resolution, which implies a more precise dijet mass measurement, is once again crucial.

\footnotetext{
${ }^{11}$ For example, the Silicon Vertex Detector will be replaced by a longer one, SVX II.
} 

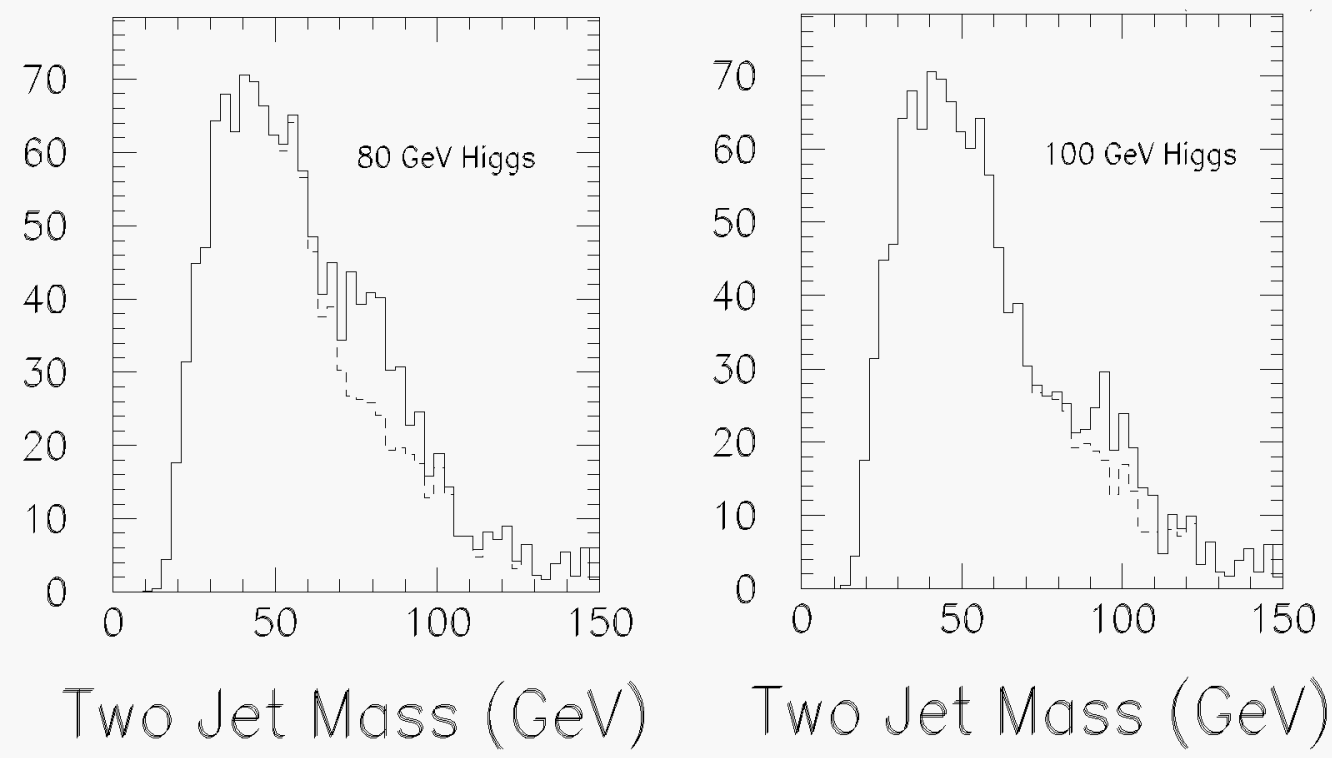

Figure 2.9: Expected signal+background mass distribution for the WH process with $10 \mathrm{fb}^{-1}$ of data at $2 \mathrm{TeV}$. The solid line is signal+background, the dashed line the sum of all backgrounds. A $30 \%$ better resolution jet energy resolution than the nominal one is assumed. 


\section{Chapter 3}

\section{The Collider Detector at Fermilab}

In this chapter a short overview of CDF detector is given. We will focus our attention on those parts of detector that are relevant to jet analysis. A full description of the complete detector can be found in [33], [34].

Calorimeters (sec. 3.4) are the most important device for jet measurement. In this thesis we will show how the tracks transverse momentum measurement performed by the Central Traking Chamber (CTC) (sec. 3.3) can help in jet energy reconstruction. For this reason a short description of the algorithm of track reconstruction will be given. Finally, the Muon detection system (sec. 3.5) and the three level trigger (sec. 3.6) will be described, since their performances are also important in our strategy aiming at an improved jet energy resolution.

\subsection{The Tevatron Collider}

The Fermilab Tevatron Collider is at present the world's highest energy particle accelerator, colliding protons and antiprotons at a center of mass energy of $1.8 \mathrm{TeV}$. The colliding ring is $6.28 \mathrm{Km}$ long. At present, two experiments, CDF and D0 (see fig. 3.1) are installed at two interaction point along the ring.

Protons and antiprotons are confined within bunches with gaussian density distribution whose width is approximately $36 \mu \mathrm{m}$ in radial direction and $590 \mathrm{~cm}$ longitudinally. In the 1992-1995 run, 6 bunches per beam were used and the time distance between two successive collisions was $3.5 \mu \mathrm{s}$. The number of events produced in a unit of time $\frac{d N}{d t}$ is proportional to cross section $\sigma$ through the luminosity $\mathcal{L}(t)$, an important parameter to characterize a collider accelerator performance: 


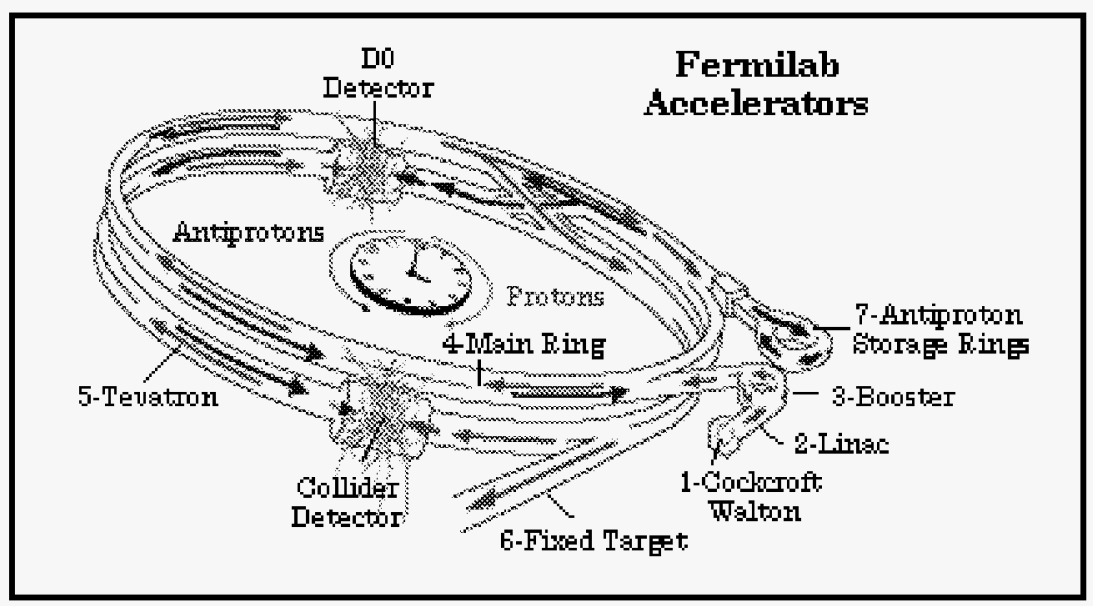

Figure 3.1: Overall view of the Tevatron Collider.

$$
\frac{d N}{d t}=\mathcal{L}(t) \sigma \quad \Rightarrow \quad N=\sigma \int \mathcal{L}(t) d t
$$

The integrated luminosity $\int \mathcal{L}(t) d t$ during a run, gives the total intensity supplied by the machine. For "RUN 1 " it was about $140 \mathrm{pb}^{-1}$ with a peak luminosity of about $2 \cdot 10^{31} \mathrm{~cm}^{-2} \mathrm{~s}^{-1}$. During data taking, CDF collected data with a typical efficiency of $80 \%$.

\subsection{CDF Detector Overview}

The goal of CDF is to measure energy, momentum and, when possible, the identity of the particles produced in $p \bar{p}$ collision. The strategy that has been chosen is to surround the interaction region with layers of different detector components, covering the entire range of azimuthal angle $\phi$ around the beam. The structure of CDF has an azimuthal and a forward/backward polar symmetry. Fig. 3.2 a cut view of the detector.

Particles coming from the interaction point encounter in sequence tracking detectors, sampling calorimeters and muon detectors. Tracks are bent by a solenoidal magnetic field of about 1.4 Tesla, generated by a superconducting coil with a $3 \mathrm{~m}$ in diameter and extending $5 \mathrm{~m}$ in length. Events are analyzed in a short time (a few microseconds) by a powerful and flexible trigger system. 


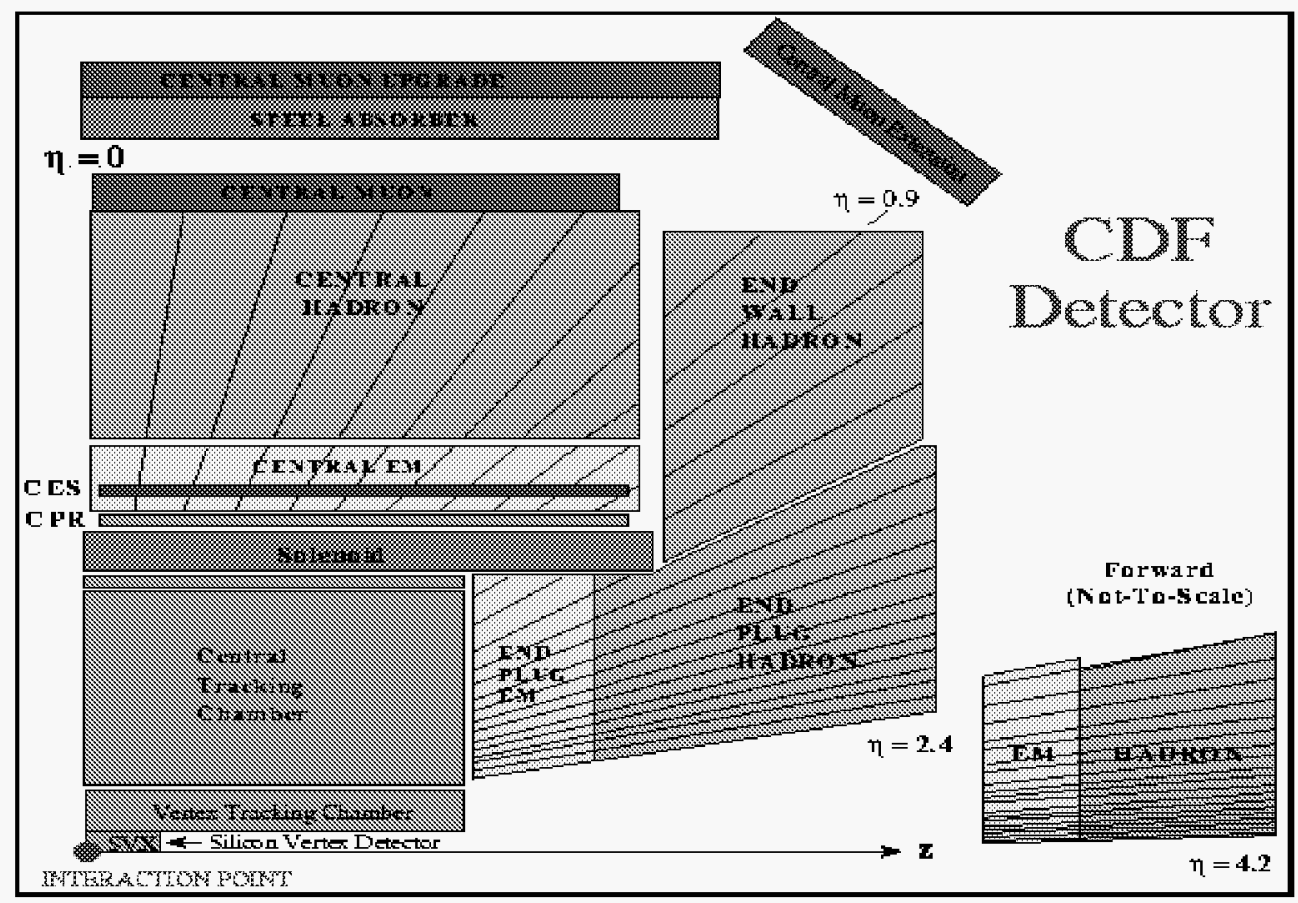

Figure 3.2: Overall view of CDF detection system.

\subsection{Tracking System}

\section{SVX, the Silicon Vertex Detector}

A four-layer silicon microstrip vertex detector (SVX) surrounds the $1.9 \mathrm{~cm}$ beryllium beampipe. SVX consists of two $51 \mathrm{~cm}$ long identical approximately cylindrical modules meeting at $z=0$. Since the $p \bar{p}$ interaction source is spread along the beampipe (the $z$ direction) with a standard deviation $\sigma \sim 30 \mathrm{~cm}$, the acceptance of SVX is just about $60 \%$ for $p \bar{p}$ interactions. The three innermost layers are at distances of $3.0,4.2$ and $5.7 \mathrm{~cm}$ from the beampipe. The microstrips are axial and have $60 \mu \mathrm{m}$ pitch, while the outermost layer is $7.9 \mathrm{~cm}$ far away from the beampipe and has a $55 \mu \mathrm{m}$ pitch. This architecture provides precision track reconstruction in the $r-\phi$ plane. The microstrip detector single-hit resolution is $\sigma=13 \mu \mathrm{m}$ and the impact parameter resolution for tracks with momentum larger than $5 \mathrm{GeV} / \mathrm{c}$ is about $\sigma=17 \mu \mathrm{m}$.

\section{VTX, the Vertex Drift Chamber}

Outside the SVX, eight vertex time projection chambers (VTX) cover the region of pseudorapidity $|\eta|<3.25$ and radial distance from the beampipe $8<r<22 \mathrm{~cm}$. The VTX provides tracking in the $r-z$ plane with $200 \div$ 
$500 \mu \mathrm{m}$ single-hit resolution and is used to measure the $p \bar{p}$ interaction vertex along the $z$ axis with a resolution of $\approx 1 \mathrm{~mm}$.

\section{CTC, the Central Tracking Chamber}

Both SVX and VTX are mounted inside the CTC, the central drift chamber covering the region $|\eta| \lesssim 1.5$ at a radial distance from the beampipe 30.9 $\mathrm{cm}<r<132 \mathrm{~cm}$. The chamber, which is $3.2 \mathrm{~m} \mathrm{long}$, is composed by 84 concentric cylindrical sense wire layers. 60 of them (axial layers) have wires parallel to the beam direction and provide $r-\phi$ tracking with $200 \mu \mathrm{m}$ resolution, while additional 24 stereo layers are tilted at \pm 3 degrees with respect to the beams. The use of both types of layers allows $r-z$ tracking at $\theta \geq 40^{\circ}$ with $4 \mathrm{~mm}$ resolution.

The track parameters $p_{T}, \eta, \phi$ are well measured by the CTC, but one needs a further step to evaluate the impact parameter $L_{x y}$. This is a very important quantity to indicate whether one is dealing with a heavy flavor jet. To accomplish this task we have to combine the CTC results with informations coming from the very inner SVX detector. Track reconstruction begins by fitting CTC hits and forcing a list of CTC candidate tracks to silicon strips hits. The algorithm used is known as "progressive method". It starts with the results of CTC fit and then updates them by adding one-by-one the SVX layers. The main steps are :

- A track reconstructed in the CTC is extrapolated back to the external layer of SVX.

- A "road" in $R-\phi$ space is defined whose width depends on the covariance matrix of the CTC fit.

- A new fit is performed using the SVX hits found inside the road. A new $\chi^{2}$ is defined and tracks above a given threshold are discarded.

- This procedure is repeated including the next SVX layer until the inner most SVX layer is reached.

A track will be defined as "SVX track" if at least two SVX clusters are added to an initial CTC track. The overall SVX-CTC system provides a track transverse momentum resolution

$$
\frac{\delta P_{T}}{P_{T}}=\sqrt{\left(0.0009 \cdot P_{T}\right)^{2}+(0.0066)^{2}}
$$

$\left(P_{T}\right.$ in $\left.\mathrm{GeV} / \mathrm{c}\right)$. The impact parameter resolution is finally

$$
\delta L_{x y}=\left(13+\frac{40}{P_{T}}\right) \mu \mathrm{m}
$$


The relevant parameters of the tracking chambers are listed in the Table 3.3.

\begin{tabular}{|c|c|c|c|}
\hline & SVX & VTX & CTC \\
\hline Coverage & $\eta \mid<1,5$ & $\eta \mid<3,25$ & $\eta \mid<1,5$ \\
\hline Inner radius $(\mathrm{cm})$ & 2,7 & 8 & 30,9 \\
\hline Outer radius $(\mathrm{cm})$ & 7,9 & 22 & 132 \\
\hline Length $(\mathrm{cm})$ & $2 \times 26$ & 280 & 320 \\
\hline Layers & 4 & 24 & $\begin{array}{c}60 \text { axial } \\
24 \text { stereo }\end{array}$ \\
\hline Space between & $60 \mu m$ (the first 3) & $6,3 \mathrm{~mm}$ & $10 \mathrm{~mm}$ \\
\hline Strips or wires & $55 \mu m$ (the others) & & \\
\hline Spatial resolution & $15 \mu m(\mathrm{r}-\phi)$ & $200-500 \mu m(\mathrm{z})$ & $\begin{array}{c}200 \mu m(\mathrm{r}-\phi) \\
4 \mathrm{~mm}(\mathrm{z})\end{array}$ \\
\hline$P_{T}$ resolution $^{a}$ & $\frac{\delta P_{T}}{P_{T}}=0,001 \cdot P_{T}(\mathrm{GeV} / \mathrm{c})$ & & $\frac{\delta P_{T}}{P_{T}}=0,002 \cdot P_{T}(\mathrm{GeV} / \mathrm{c})$ \\
\hline Thickness (at $\left.\theta=90^{\circ}\right)^{b}$ & $\sim 0,035 \mathrm{X}_{0}$ & $\sim 0,045 \mathrm{X}_{0}$ & $\sim 0,015 \mathrm{X}_{0}$ \\
\hline
\end{tabular}

${ }^{a}$ For SVX, CTC informations are included

${ }^{b} \mathrm{X}_{0}$ is the radiation length for electrons

Table 3.1: Parameters of the CDF tracking detectors.

\subsection{Calorimeters}

CDF employs sampling calorimeters. Layers of sampling material are interleaved with layers of absorber in a sandwich fashion. Incoming primary particles produce showers of secondary particles in the absorber. The showers deposit a fraction of their energy in the sampling material, which produces a signal which is recorded and summed over all sampling layers. A calibration based on test beam data is then applied to derive the particle energy.

Calorimeters are segmented in azimuth and pseudorapidity to form a projective tower geometry, pointing back to the nominal interaction point. Each tower has an electromagnetic compartment in front of a corresponding hadronic calorimeter, so that we can make a comparison of electromagnetic to hadronic energy on a tower-by-tower basis.

By measuring the energy deposited in a projective tower by a particle, we also simultaneously measure the angle at which the particle emerged from the interaction point.

Ranging from $\theta=90^{\circ}$ down to the beam axis, there are three separate calorimeters, called central, plug and forward. Each of them covers the entire $2 \pi$ azimuthal angle. All towers are 0.1 wide in $\eta$. The central towers are $15^{\circ}$ wide in $\phi$. Plug and forward towers are only $5^{\circ}$ wide. This segmentation 
is fine enough that jets will normally spread over more than one tower. This coverage is resumed in Table 3.4 .

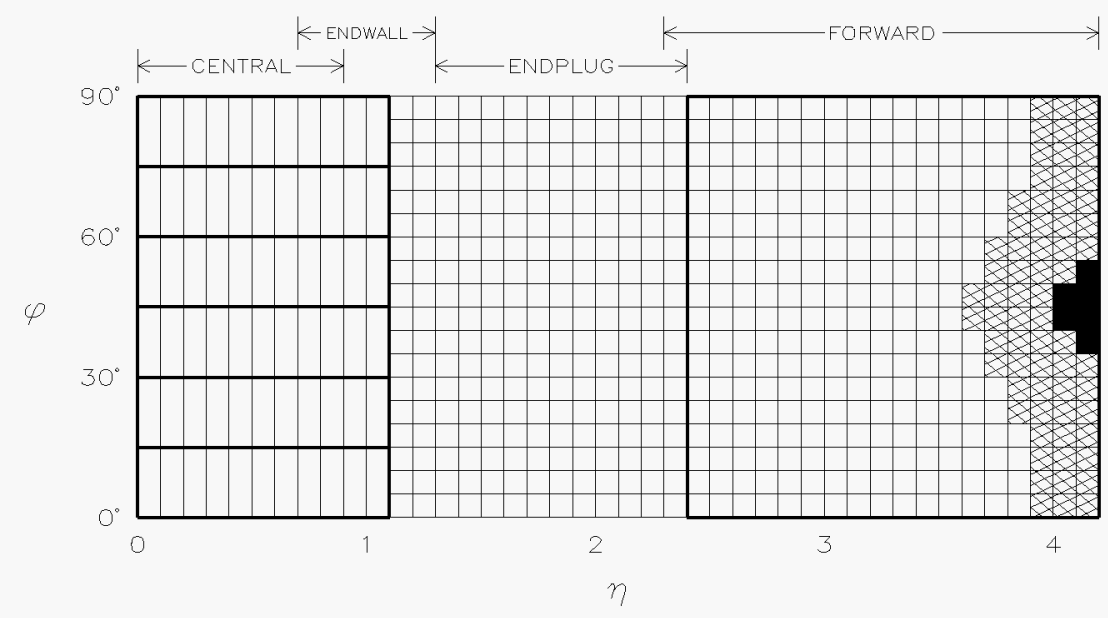

Figure 3.3: Coverage of the calorimeter towers in $\eta-\phi$ space.

The calorimeters are of two types. The Central is a scintillator calorimeter, while the Plug and Forward are gas calorimeters. Scintillator was chosen in the central region for its good resolution. Closer to the beam the towers are smaller in $\theta$ - to maintain a fixed $\eta$ - making the construction of a scintillator calorimeter less practical. Gas calorimeters are easily segmented into small towers using pads in the cathode plan, and robustly withstand high multiplicities, making them a natural choice for the forward region. Unfortunately, they have a significant worse resolution than scintillator calorimeters. This is one reason why in this thesis only central jets are taken into account. ${ }^{1}$

\section{Central Calorimeter}

The Central calorimeter is azimuthally arranged in 48 physically separated $15^{\circ}$ wide modules called wedges - 24 wedges at positive $z$ and likewise at negative.

Each wedge is segmented into ten towers in $\eta$ - the segmentation of the whole calorimeter is illustrated in Fig 3.3).

Each tower in the central is composed by a Central Electromagnetic Calorimer (CEM) backed by a Central Hadronic Calorimeter (CHA).

\footnotetext{
${ }^{1}$ For Run II, a refined technology has been developed allowing an extended plug calorimeter to be assembled with projective plastic scintillator towers. The new plug will replace the old Plug/Forward calorimeter system.
} 
The hadronic compartment is completed by the Endwall Hadron Calorimeter (WHA) that extends the extends the coverage of the central calorimeter down to $30^{\circ}(\eta=1.3)$.

Proportional chambers are located between the solenoid and the CEM forming the Central Preradiator Detector (CPR) which provides $r-\phi$ information on electromagnetic shower initiating in the solenoid coil.

Located six radiation lengths deep in the CEM calorimeters (approximately at shower maximum) is the Central Electromagnetic Strip detector (CES). These are proportional chambers with orthogonal wires and inductive strips, that measure the EM shower position, both in $R-\phi$ and $z$.

The $\phi$ boundaries between the wedges are uninstrumented regions were the response is not flat ( $\phi$ cracks). The boundary between the two halves of the central calorimeter constitutes one of the main uninstrumented regions (at about $\theta=90^{\circ}$ ). The steel and gap between the wedge and endwall modules constitute another region of complicated response.

\section{End Plug and Forward Calorimeters}

These calorimeters contain a mixture of $50 \%$ argon, $50 \%$ ethane with a small percentage of alcohol to prevent glow discharge.

They are subdivided in several components (see fig. 3.2):

- Endplug Electromagnetic Calorimeter (PEM);

- Endplug Hadronic Calorimeter (PHA);

- Forward Electromagnetic Calorimeter (FEM);

- Forward Hadronic Calorimeter (FHA).

\subsection{Muon Detection}

The central calorimeters act as hadron absorber for the central muon (CMU) detection system, which consists of four layers of drift chambers located behind them. The CMU covers about the $84 \%$ of the solid angle for $|\eta|<0.6$ and can be reached by muons with $P_{T}$ larger than $1.4 \mathrm{GeV} / \mathrm{c}$. In 1992, 0.6 $m$ of steel was added behind the CMU system for additional hadron absorption, and behind the steel the central muon upgrade detector (CMP) was added, consisting of additional four layers of drift chambers covering about $63 \%$ of the solid angle for $|\eta|<0.6$. Only about $53 \%$ of the solid angle corresponding to $|\eta|<0.6$ is covered by both CMU and CMP. To extend 


\begin{tabular}{lccc}
\hline \hline System & $\eta$ range & Resolution & Thickness \\
\hline CEM & $|\eta|<1.1$ & $13,7 \% / \sqrt{E_{T}} \oplus 2 \%$ & $18 \mathrm{X}_{0}$ \\
PEM & $1.1<|\eta|<2.4$ & $22 \% / \sqrt{E_{T}} \oplus 2 \%$ & $18-21 \mathrm{X}_{0}$ \\
FEM & $2.2<|\eta|<4.2$ & $26 \% / \sqrt{E_{T}} \oplus 2 \%$ & $25 \mathrm{X}_{0}$ \\
CHA & $|\eta|<0.9$ & $50 \% / \sqrt{E_{T}} \oplus 3 \%$ & $4,5 \lambda_{0}$ \\
WHA & $0.7<|\eta|<1.3$ & $75 \% / \sqrt{E_{T}} \oplus 4 \%$ & $4,5 \lambda_{0}$ \\
PHA & $1.3<|\eta|<2.4$ & $106 \% / \sqrt{E_{T}} \oplus 6 \%$ & $5,7 \lambda_{0}$ \\
FHA & $2.4<|\eta|<4.2$ & $137 \% / \sqrt{E_{T}} \oplus 3 \%$ & $7,7 \lambda_{0}$ \\
\hline \hline
\end{tabular}

Table 3.2: Angular coverage and resolution of the CDF calorimeters. $X_{0}=$ electron radiation length, $\lambda_{0}=\pi^{+}$interaction length, $E_{T}$ in $G e V$.

the pseudorapidity region covered by the muon detection system, four freestanding conical drift chambers, sandwiched between scintillator counters for triggering, have been added in order to cover about $71 \%$ of the solid angle of the region $0.6<|\eta|<1.0$. This additional system is called the central muon extension (CMX).

The central muon chambers measure four points along the trajectory with an accuracy of $250 \mu \mathrm{m}$ per point in the $\phi$ direction. Charge division gives an accuracy of $\sigma=1.2 \mathrm{~mm}$ per point in the $z$ direction. The system is about $100 \%$ efficient for muons within the covered solid angle when the muon momentum is above $3 \mathrm{GeV} / \mathrm{c}$. Muons are matched both in position and angle to tracks in the CTC where their transverse momentum is measured. In both the forward and backward regions there is a muon spectrometer consisting of large magnetized steel toroids backed by drift chamber planes and triggering counters. The angular region covered by each spectrometer lies between $3^{\circ}$ and $16^{\circ}$ from each beam line. The momentum resolution is $13 \%$, independent of momentum, for muons with total momentum $P>8$ $\mathrm{GeV} / \mathrm{c}$. The drift chambers measure the muons trajectory with an accuracy of $5^{\circ}$ in the $\phi$ direction, and $\sim 200 \mu \mathrm{m}$ in the $r$ direction.

\subsection{Trigger}

The CDF trigger is a three level system.

\section{Level 1}

Level 1 uses fast outputs coming from the muon chambers for muon triggers and from the calorimeters for electrons and jets triggers. Both hadronic and 
electromagnetic calorimeter towers are summed into trigger towers with a width in pseudorapidity $\Delta \eta=0.2$ and a width in azimuth $\Delta \phi=15^{\circ}$. The trigger signals from the calorimeters are sent to the trigger electronics and separately stored until a level 1 decision is made. If level 1 is not satisfied in a given crossing, a reset will automatically be sent to clean the login in time for the next beam crossing, so no deadtime is introduced by events which do not pass level 1. Level 1 calorimeter triggers require the sum of $E_{T}$ for all calorimeter towers which are individually greater than a low threshold (typically $1 \mathrm{GeV}$ ) to be greater than a higher threshold (typically $30 \div 40$ $\mathrm{GeV}$ ). At a typical luminosity of $5 \cdot 10^{30} \mathrm{~cm}^{-2} \mathrm{~s}^{-1}$ the rate of level 1 triggers is about $1 \mathrm{kHz}$.

\section{Level 2}

The level 2 trigger starts after a level 1 trigger has been satisfied. A hardware cluster finder searches for clusters of energy. Towers below a programmable threshold are ignored. This operation takes about 200 ns per cluster. The energy of all towers identified as belonging to the same cluster are summed to form total $E_{T}$ and $E_{T}$-weighted first and second moments of the clusters. Separate sums are kept for hadronic and electromagnetic energy. Each cluster is sorted in a list with its $E_{T}, \eta$ and $\phi$ informations. A match is made between the clusters and CTC tracks provided by a fast $(10 \mu s)$ hardware tracking processor (CFT). CFT tracks can be matched to CMU, CMP or CMX segments too, to make candidate muons. Clusters with electromagnetic energy contents are matched to CFT tracks in order to make candidate electrons. The final trigger is a selection on muons, electrons, photons, jets and missing $E_{T}$. At a $5 \cdot 10^{30} \mathrm{~cm}^{-2} \mathrm{~s}^{-1}$ luminosity, the level 2 trigger output rate is about $12 \mathrm{~Hz}$.

\section{Level 3}

Events selected in level 2 trigger are read out into commercial processors and submitted to reconstruction software algorithms identical to those used in the "off-line" analysis. Most of the execution time is used for the threedimensional track reconstruction in the CTC. Those events passing this filter algorithm are stored on magnetic tape for off-line processing with about 5 $\mathrm{Hz}$ output rate. 


\section{Chapter 4}

\section{Jets at CDF}

As we have discussed in the first chapter, jets are a frequent product of a high energy $p \bar{p}$ interaction and its measurement is crucial in the analysis of data collected in an hadron collider. It has been shown in the second chapter how the accuracy with which the jets can be measured rules the error in several studies performed in Run I and how new physics could be discovered in Run II if a better jet reconstruction will be accomplished.

In this chapter we shall discuss how jet reconstruction was done at CDF so far pointing out sources of jets mis-measuring. In the following chapters the whole matter will be reappraised looking for an alternative way to improve jet reconstruction at $C D F$.

\subsection{Jet Clustering and Jet Energy Definition}

In this section the CDF jet clustering is described. A more extensive discussion on possible "best" jet algorithms will be given in the next chapter.

\subsubsection{JETCLU, the CDF Jet Cluster Algorithm}

The CDF calorimeter cells (towers) form the basic units of the clustering algorithm. An off-line routine (JETCLU) assigns calorimeter towers to clusters in three steps [35]:

1. Preclustering Plug and forward calorimeter towers are merged into "seed towers" of a common $\Delta \phi$ segmentation of $15^{\circ}$. Central towers are kept unchanged. Then a search is made for seed towers above threshold $\left(E_{T_{S E E D}} \geq 1.0 \mathrm{GeV}\right)$. A list is made with seed towers sorted in order of decreasing $E_{T}$. A loop is performed to group seeds into preclusters. A seed tower is added to a precluster if it is within a preset distance $R_{0}$ in 
$\eta-\phi$ space of the largest $E_{T}$ tower in the precluster and it is adjacent to a seed tower already assigned to the precluster. If a seed tower cannot be assigned to an existing precluster, it starts a new precluster. The list of resulting preclusters is passed to the next step.

2. Cone algorithm The centroid of each precluster is defined as the $E_{T}$ weighted $\eta$ - $\phi$ centroid of its towers. A loop is performed around the centroid over towers with $E_{T}$ above a low threshold $\left(E_{T_{M I N}}=0.1 \mathrm{GeV}\right)$, adding them into a cluster if they are within $R_{0}$ of the centroid. $R_{0}$ is the so called "cone radius" which is chosen to fit each particular analysis best. The most common values are $0.4,0.7$ and 1.0. After this loop, the centroid is recalculated taking into account the new towers now assigned to the cluster. If the list of towers of the cluster has changed, the loop over towers is repeated using the new centroid. For each cluster this procedure is repeated until the list is unchanged in two consecutive passes.

3. Merge and/or resolve overlaps The cone algorithm is such that some towers may emd in being assigned to more than one cluster. As a last step, overlapping towers are either separated, or the entire clusters are merged into one. If the amount of overlapping energy is higher than some preset value - usually the $75 \%$ of the smallest cluster the clusters are merged. If the overlapping energy is lower than the threshold, the shared towers are assigned to the nearest cluster. After clusters have been separated or merged, the centroid of each cluster is recalculated using the new list of towers. 


\subsubsection{Jet Energy and Momentum}

The jet energy and momentum components are defined from the list of cluster towers as:

$$
\begin{aligned}
E_{J} & =\sum_{i=1}^{N} E_{i} \\
P_{x, J} & =\sum_{i=1}^{N} E_{i} \sin \theta_{i} \cos \phi_{i} \\
P_{y, J} & =\sum_{i=1}^{N} E_{i} \sin \theta_{i} \sin \phi_{i} \\
P_{z, J} & =\sum_{i=1}^{N} E_{i} \cos \theta_{i}
\end{aligned}
$$

where $i$ is the tower index and $N$ the number of towers in the cluster. The angles $\theta_{i}$ is calculated with respect to the event vertex along the beam axis. The angle $\phi$ is measured relative to a preset reference ( $\phi=0$ on the horizontal plane). Note that, by these definitions, jets are not massless.

Using the above defined quantities, the jet energy and momentum transverse components $P_{T, J}$ and $E_{T, J}$ are derived as:

$$
\begin{aligned}
P_{T, J} & =\sqrt{P_{x, J}^{2}+P_{y, J}^{2}} \\
P_{J} & =\sqrt{P_{x, J}^{2}+P_{y, J}^{2}+P_{z, J}^{2}} \\
E_{T, J} & =E_{J} \frac{P_{T, J}}{P_{J}}
\end{aligned}
$$

\subsection{Jet Energy Corrections}

To reconstruct the true momentum of parton from the measured jet $E_{T}$ inside a clustering cone, proper corrections need to be applied to account for detector and physics effects. The corrections include:

- Relative correction: correction for non-uniform response of different calorimeters and the effects of gaps and edges in the calorimeters.

- Absolute correction: estimate of the true parton $E_{T}$ inside the cone based on the observed raw $E_{T}$, accounting for non-linear response of the calorimeter to low momentum particles and invisible fragmentation in the tracking and calorimetry detectors. 
- Underlying event (UE) subtraction: subtraction of the estimate $E_{T}$ inside the clustering cone produced by remnants of the $p \bar{p}$ system not involved in the hard scattering.

- Out-Of-Cone (OOC) addition: inclusion of the un-measured $E_{T}$ belonging to the jet but emitted outside the clustering cone, estimated using fragmentation model.

The correction is done in three stages. Firstly the relative correction is applied and then the absolute one is performed. Finally, out-of-cone and underling event corrections are included. All these corrections are implemented by the off-line routine JTC96. From now on the standard jets corrections will be referenced as "JTC96 corrections".

The method used to extract each correction will be now described.

\subsubsection{Relative Corrections}

Particles falling into cracks release energy which is partially detected, so a relative correction is applied to normalize it the response of fully efficient areas.

As a function of pseudorapidity $\eta$, this process results $[36,37]$ in correcting jets back to an equivalent jet in the $\eta$ range $0.2 \leq|\eta| \leq 0.7$, where the calorimeter response is flat and non-linearities are well understood from extensive test-beam measurements.

In a perfect detector, dijet events should balance back-to-back in $P_{T}$. This suggests to use jet balancing to construct the relative jet correction, by requiring the $P_{T}$ of a jet in the central well instrumented region to be equal to the $P_{T}$ of the jet outside this region. Events are required to pass the following cuts:

- at least 1 jet ("trigger jet") at $0.2 \leq|\eta| \leq 0.7$;

- one additional jet ("probe jet") with $P_{T}>15 \mathrm{GeV} / \mathrm{c}$;

- no third jet with $P_{T}>15 \mathrm{GeV} / \mathrm{c}$;

- $z$ coordinate of the event vertex $|z| \leq 60 \mathrm{~cm}$;

- no other vertex in the event;

- azimuthal distance between the two jets $\Delta \phi \geq 2.5$ radians. 
These cuts define the dijet sample used to get the relative jet correction . In the following, the central jet is supposed to be the "trigger" while the other is the "probe" jet.

We call missing $E_{T}$ projection fraction (MPF) the ratio between the missing $E_{T}\left(\mathbb{Z}_{\mathrm{T}}\right)$ projection along the $P_{T}^{\text {Probe }}$ direction $\left(\widehat{P}_{T}^{\text {Probe }}\right)$ and the mean of $P_{T}^{P r o b e}$ and $P_{T}^{\text {Trigger }}$ :

$$
M P F=\frac{2\left(\mathbb{E}_{\mathrm{T}} \cdot \widehat{P}_{T}^{\text {Probe }}\right)}{P_{T}^{\text {Probe }}+P_{T}^{\text {Trigger }}}
$$

Since, in the $\mathrm{Z}_{\mathrm{T}} \ll P_{T}^{\text {Probe }}$ hypothesis,

$$
\nexists_{\mathrm{T}} \cdot \widehat{P}_{T}^{\text {Probe }} \simeq P_{T}^{\text {Trigger }}-P_{T}^{\text {Probe }}
$$

we can write

$$
M P F=\frac{2\left(P_{T}^{\text {Trigger }}-P_{T}^{\text {Probe }}\right)}{P_{T}^{\text {Trigger }}+P_{T}^{\text {Probe }}}
$$

Then, defining the relative jet scale correction factor $\beta$ as

$$
\beta=\frac{P_{T}^{\text {Trigger }}}{P_{T}^{\text {Probe }}}
$$

we get

$$
\beta=\frac{2+M P F}{2-M P F}
$$

which, in general, depends on $P_{T}$ and $\eta$. What we are searching for is a relative correction function for each value of $P_{T}$ and $\eta$, and this can be done by fitting the $\beta$ distribution with respect to $\eta$ using a continuous curve at fixed $P_{T}^{\text {Trigger }}[37]$ (Fig. 4.2.1).

\subsubsection{Absolute Corrections}

An "absolute correction" is needed in the attempt to transform the jet energy into the original parton energy. In general, parton energies are largely underestimated by the relative-corrected calorimeter response because of energy leakage or nuclear absorption. Monte Carlo simulations are used in this process by following this path:

- one parton and a jet are associated if their directions both fall in a matching cone;

- the jet $P_{T}$ is evaluated summing the $P_{T}$ of all particles that fall into the matching cone; 


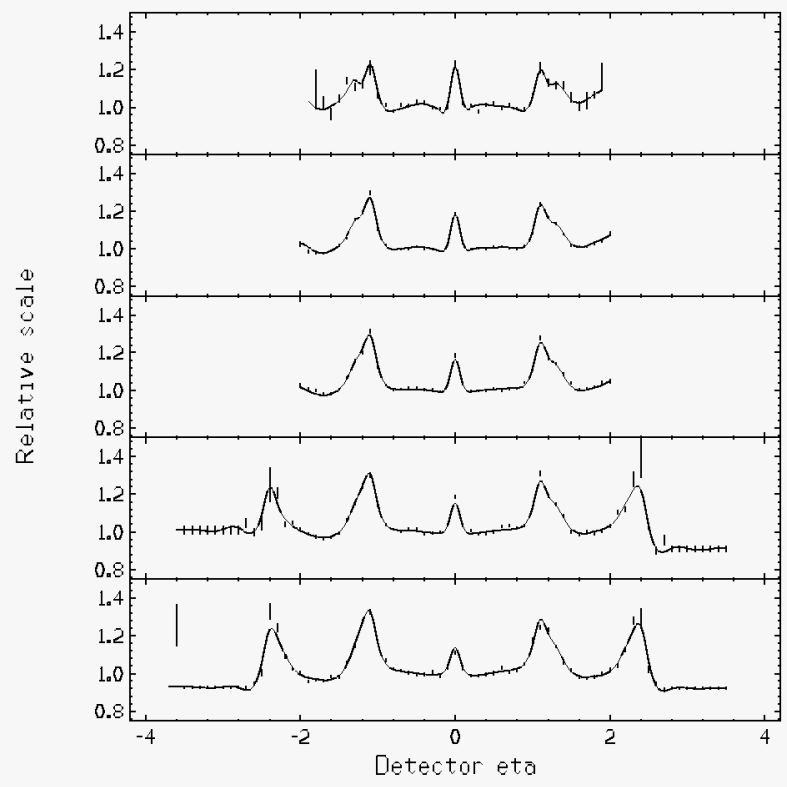

Figure 4.1: Relative corrections for Run 1B. Each slice refers to a fixed $\mathrm{P}_{\mathrm{T}}$ (trigger) (from top to bottom 191.5, 120.5, 86, 62.5 and 32 $\mathrm{GeV} / \mathrm{c}$ ). The effect of "cracks" is evident: at $\eta=0$ between central calorimeters, at $|\eta|=$ 1,2 between central and plug calorimeters, and at $|\eta|=2,4$ between plug and forward calorimeters [37].

- a $P_{T}$ dependent correction factor is defined as the ratio between parton $P_{T}$ and jet $P_{T}$ :

$$
\alpha\left(P_{T}\right)=<\frac{P_{T}^{\text {Parton }}}{P_{T}^{J e t}}>
$$

This procedure gives a correction that depends on the detector response and the $P_{T}$ spectrum in the process. In the QFL simulation package of CDF, the detector response was calibrated with CTC isolated tracks. A Monte Carlo routine (SETPRT) - tuned on CDF data for tracks of transverse momentum larger than $1 \mathrm{GeV} / \mathrm{c}$ - takes care of the parton fragmentation into jets.

The absolute corrected energy is affected by an uncertainty due to calorimeter response calibration, fragmentation model and underlying event effects. This overall uncertainty is parametrized by the so-called "Behrends curves" (Fig. 4.2). 


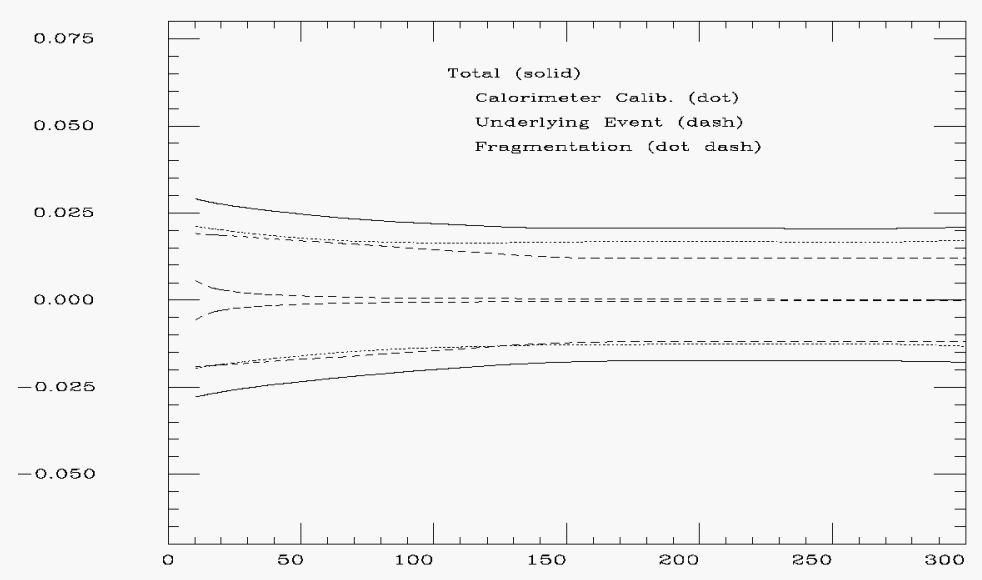

Figure 4.2: Absolute corrections: Behrends curves. Relative uncertainty on jet energy after relative and absolute corrections as a function of the energy in GeV.

\subsubsection{Underlying Event Subtraction}

The underlying event corrections (UE) are an attempt to take into account tracks not belonging to the jet, but falling inside its cone. In "minimum bias" events $^{1}$ the $\sum E_{T}$ over the calorimeter towers in the region $-1.0 \leq \eta \leq 1.0$ is computed and normalized to its coverage. This gives the energy density to be used for the correction ${ }^{2}$. Since the underlying energy correction should take care also of particles coming from other interactions in the same bunch crossing (multiple vertices), it is next parametrized to the number of vertices found by VTX in the same bunch crossing (see Table 4.2.3). The different behaviour of this correction in Run $1 \mathrm{~A}$ and Run 1B, which is shown by Table 4.2 .3 , is due to the fact that the average number of superimposed events in Run $1 \mathrm{~A}$ was about 2 , while in Run $1 \mathrm{~B}$ was about 3.

As Table 4.2.3 shows, no $P_{T}^{\text {Parton }}$ dependence is observed for the energy density factor.

\subsubsection{Out-of-Cone Addition}

The out-of-cone (OOC) corrections take into account the fact that, due to gluon emission, some particles produced in the jet fragmentation process fall outside the cluster cone and therefore are not included when reconstructing the jet energy.

\footnotetext{
${ }^{1}$ Events filtered by a trigger just demanding the occurrence of a collision.

${ }^{2}$ Since this density is evaluated at calorimeter level, it is necessary to multiply it by the absolute correction factor to get the correction at parton level.
} 


\begin{tabular}{lllll}
\hline \hline Clustering cone & 0,4 & 0,7 & 1,0 \\
\hline UE for Monte Carlo & 0,370 & 1,133 & 2,312 \\
UE for Run 1A (data) & 0,720 & 2,210 & 4,510 \\
UE for Run 1B (data) & (a) & $0,297 \cdot \mathrm{N}$ & $0,910 \cdot \mathrm{N}$ & $1,858 \cdot \mathrm{N}$ \\
& (b) & 0,65 & 1,98 & 4,05 \\
OOC, data and Monte Carlo & $1,95+, 156 \cdot P_{T}$ & $1,29+, 051 \cdot P_{T}$ & $0,54+, 022 \cdot P_{T}$ \\
\hline \hline
\end{tabular}

Table 4.1: Underlying event and Out-Of-Cone corrections (1996). All values are given in $\mathrm{GeV} . N$ stays for $N_{v}-1$ where $N_{v}$ is the number of vertices found

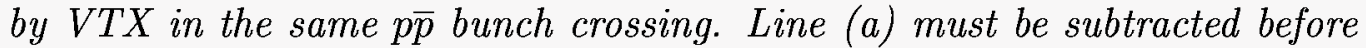
absolute correction, line (b) after them and after (a) corrections.

The study of cone losses is performed using the same Monte Carlo sample used to evaluate the absolute jet energy scale. The correction is defined as

$$
\triangle P^{O O C}\left(P_{T}, R\right)=P_{T}^{\text {Parton }}-P_{T}^{\text {Jet }}
$$

where $P_{T}^{\text {Parton }}$ is given by the sum of the $P_{T}$ of all particles coming from the parton and $P_{T}^{\text {Jet }}$ is given by the sum of the $P_{T}$ of all particles coming from the parton and falling inside the cone. $\triangle P^{O O C}$ is linearly parametrized in $P_{T}$, as shown in Table 4.2.3.

\subsubsection{Specific Corrections}

Sometimes the JTC96 corrections just described must be improved when exclusive physics process are addressed. For example, analysis of HERWIG $t \bar{t}$ Monte Carlo samples have shown significant [38] disagreements between reconstructed and primary parton energies. That could be traced to the inclusive JTC96 corrections being inadequate for jets produced in that specific process. Hence, for the top mass analysis, an additional set of jet corrections [38] is applied after JTC96 to bring the jet energy to agree with the HERWIG parton energy. These corrections (named "AA corrections"), are estimated separately for

1. generic $b$-jet;

2. $b$-jet containing a semileptonic $b \rightarrow \mu \nu X$ decay;

3. $b$-jet containing a semileptonic $b \rightarrow e \nu X$ decay;

4. Quark-jets from $\mathrm{W}$ boson decay. 
If $P_{T}^{J T C}$ is the transverse momentum after JTC96, the transverse energy after AA corrections can be parametrized as

$$
P_{T}^{A A}=A A\left(P_{T}^{J T C}\right) \cdot P_{T}^{J T C}
$$

where

$$
A A\left(P_{T}^{J T C}\right)=e^{\left(p_{1}+p_{2} \cdot P_{T}^{J T C}\right)}+p_{3}
$$

$p_{1}, p_{2}$ and $p_{3}$ are calculated with a fit method. Returned values are listed in Table 4.2.5.

\begin{tabular}{lrrr}
\hline \hline jet type & $p_{1}$ & $p_{2}$ & $p_{3}$ \\
\hline generic b & $+0,14400$ & $-0,046828$ & $-0,070058$ \\
$\mathrm{~b} \rightarrow \mathrm{e} \nu \mathrm{X}$ & $+0,33470$ & $-0,041225$ & $-0,016902$ \\
$\mathrm{~b} \rightarrow \mu \nu \mathrm{X}$ & $+0,36333$ & $-0,029835$ & $+0,030716$ \\
$\mathrm{~W}$ decay & $-0,84931$ & $-0,047497$ & $-0,087614$ \\
\hline \hline
\end{tabular}

Table 4.2: Fitted parameters of AA corrections. Up to now AA corrections are available only for jets clustered within a $R(\eta, \phi)=0,4$ cone radius [38]. Thus is the jet clustering radius adopted for the top studies. 


\section{Chapter 5}

\section{Particle Level Study and Jet Algorithm}

In this chapter we shall begin to describe the work made in order to the dijet mass resolution. As a first step we have studied the process $W \rightarrow q \bar{q}$ at the particle level with the PYTHIA and HERWIG Monte Carlos ${ }^{1}$. To simplify this kind of study new event display and event reconstruction tools were developed (described in sec. 5.2.1). After having introduced the main physics effects (sec. 5.2), the out of cone correction will be revisited (sec. 5.3) and alternative jet clustering algorithms will be compared (sec. 5.4.1 and sec. 5.6) to the CDF standard algorithm (see sec. 4.1.1). A study of the best cone size will be presented (sec. 5.4.2) and a method for merging jets coming from the same parton will be described (sec. 5.5). Finally we will consider the impacts on dijet mass reconstruction of applying a cut on extra jets (sec. 5.7) in the event.

\subsection{Introduction}

In chapter 2 it was shown as jet energy resolution is the main sources of uncertainties on the dijet mass reconstruction for several processes. It is important to stress the fact that the standard approach to jet reconstruction is to buold clusters before any correction. Only after clusterization the energy of the whole jet is corrected. Only the raw energy and the eta coordinate of the jet are needed to define the ultimate value of the jet energy.

The approach used in this thesis is different. We consider separately jet reconstruction uncertainties coming from "physics" which would be present also if the energy of each particle in the jet would be exactly known, and uncertainties due to the detector resolution. In other words, we will group

\footnotetext{
${ }^{1} \mathrm{~A}$ short description of these two Monte Carlo event generators is supply in 1.6.
} 
these sources into two categories: 1) detector effects such as calorimeter resolution, and 2) algorithm effects such as fluctuations in the energy outside a clustering cone.

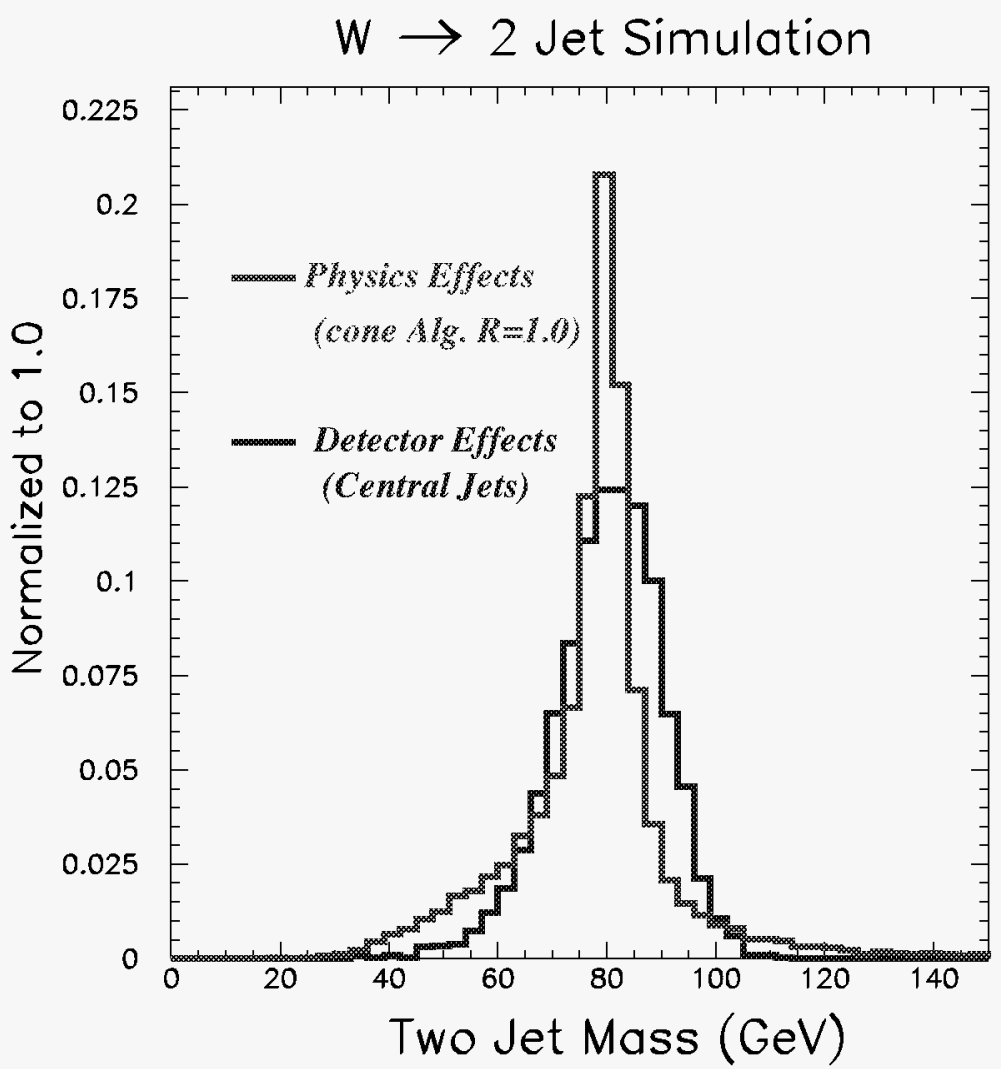

Figure 5.1: The $W \rightarrow q \bar{q}$ mass distribution with pure algorithm effects (solid), and pure detector effects (dashed)

This is shown in Fig. 5.1, where both histograms come from a $W \rightarrow$ $q \bar{q}$ simulation. The solid histogram is the particle level mass distribution using a cone algorithm with a radius 1.0 , no detector effects included. The dashed histogram is the mass distribution with only CDF detector effects, the algorithm effects are removed by using the known particle list. One can see that the two distributions are quite different, with the detector effects tending to dominate the central core of the distribution (9 GeV compared to $4 \mathrm{GeV}$ ), while the algorithm effects dominate the tails. Thus both categories of resolution will be addressed in detail starting to study algorithm effects in a general way in the present chapter. The next section will be devoted to detector effects. Algorithm effects are very physics-dependent, thus we start first analyzing this kind of effects. 


\subsection{Physics Effects}

Although algorithm effects are physics-dependent, our study will be performed in a typical peak-hunting case: $W \rightarrow$ jet jet ${ }^{2}$. In this section we will consider four different physics effects that contribute to di-jet mass resolution. They are:

1. the natural width of the $W$

2. underlying event fluctuations

3. final state gluon radiation (FSR)

4. misidentified jets from initial state gluon radiation (ISR)

The role of the underlying event was already mentioned in sec. 4.2.3, and FSR and ISR mechanisms are explained in detail in sec. 1.4.1.

Fig. 5.2 shows the effect of the natural width of the W with FSR turned off. In some cases this natural width can be the dominant source of tails. Since we are only interested in jet algorithm effects, we will turn off the W width in Pythia for all remaining plots in this chapter.

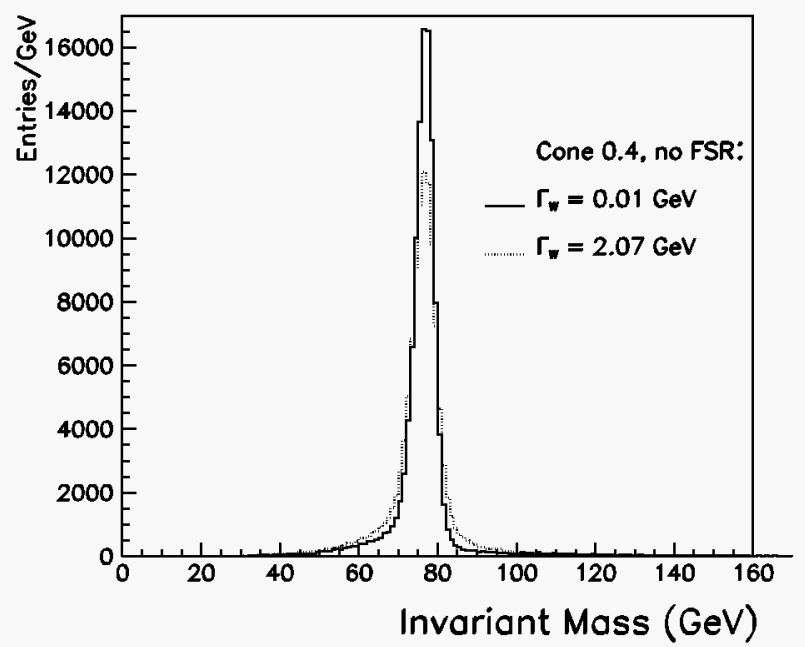

Figure 5.2: The di-jet invariant mass distribution assuming no final state radiation. The dashed curve is with the nominal $W$ width, and the solid curve is with the $W$ width set to zero.

After removing the $\mathrm{W}$ width, the main cause of the width in fig. 5.2 are the underlying event fluctuations. They mainly give a gaussian contribution with a width $\approx 2 \mathrm{GeV}$. Since the nominal gaussian width from detector

\footnotetext{
${ }^{2}$ The study of this sample can be easily extended to $H \rightarrow b \bar{b}$ process.
} 
effects (discussed earlier) is much larger than this, the underlying event fluctuations will not be studied in detail in this note.

Fig. 5.3 shows, after setting the $W$ width to zero, the effect of FSR. It is the dominant physics effect to be considered, and it is also the effect that is universal to all analyses. Therefore this will be the emphasis of this chapter.

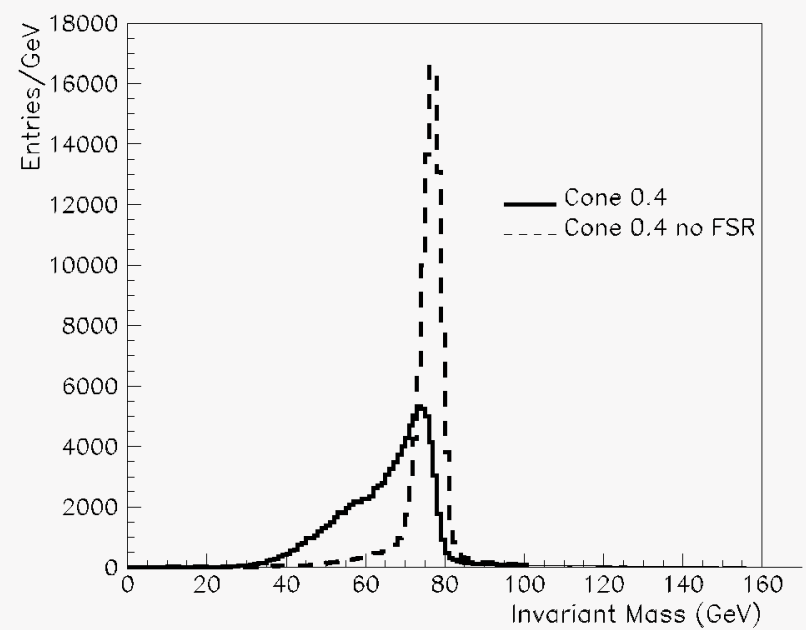

Figure 5.3: The di-jet invariant mass for cone 0.4. The solid curve is the nominal mass distribution, while the dashed curve is with FSR turned off, with the $W$ width set to zero in both curves.

The final physics effect to be considered is misidentification of the main jets from the $W$. To illustrate this we select the reconstructed events with mass $>100 \mathrm{GeV}$, and then use the Pythia particle lists to determine what is the fraction of energy carried by the two $\mathrm{W}$ decay jets. We plot the minimum of this energy (fig. 5.4). It appears that in no case the two leading jets carry most of the energy. One may deduce that in most cases one of the two leading jets is from ISR, not from the W. This can be reduced by either increasing the jet Pt cutoffs, or decreasing the jet $\eta$ limits. Since this is very physicsanalysis dependent we have not pursued this further, but will concentrate on the universal issue of final state radiation.

Finally there is always the issue of Monte Carlo dependence of the radiation. In the section 1.5 the different hadronization method used by different Monte Carlo are outlined. Ee have compared HERWIG and PYTHIA and found identical mass distributions for cone 0.4, as shown in fig. 5.5.

In the rest of the chapter we will show results obtained using PYTHIA. 


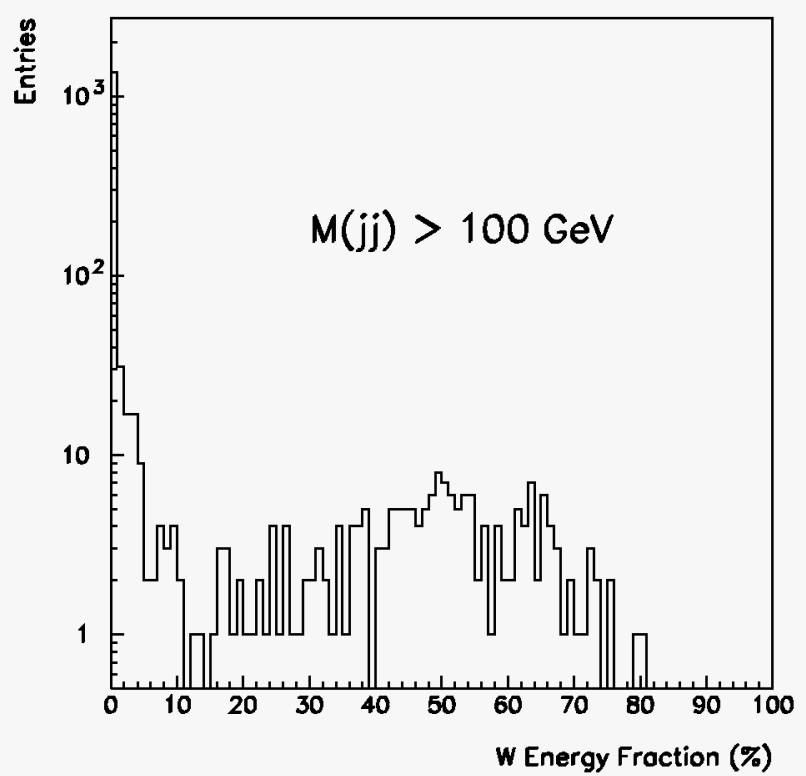

Figure 5.4: Minimum $W$ energy fraction of the two leading jets for the reconstructed events with mass $>100 \mathrm{GeV}$.

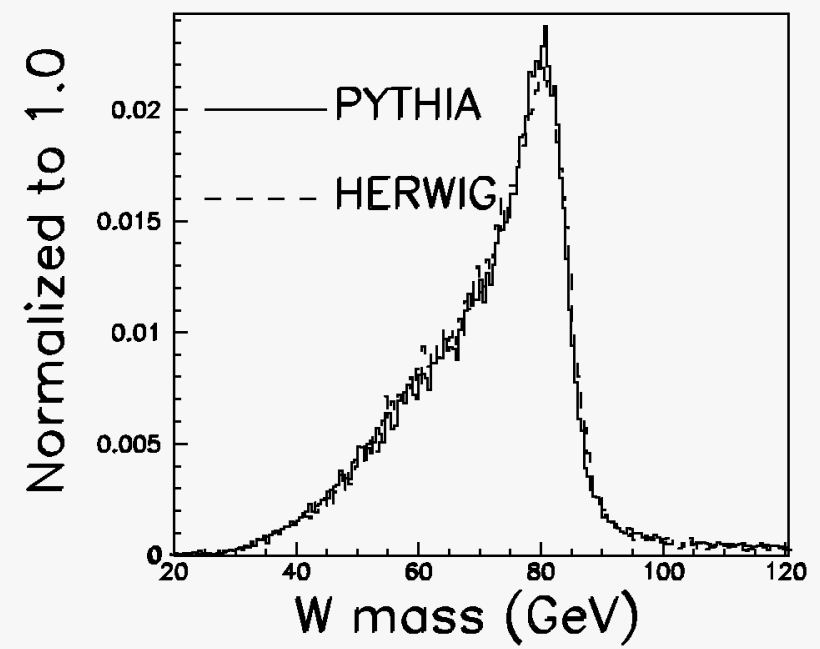

Figure 5.5: Invariant mass distribution using a cone 0.4 for PYTHIA and HERWIG.

\subsubsection{Reconstruction method and Sample Selection}

For the the analysis of physics effects we have developed some reconstruction tools to perform a particle level study. On Monte Carlo events the main steps in our reconstruction are : 
- Events are generated with a Monte Carlo (PYTHIA 5.7 or HERWIG 5.9) which accounts for the matrix element of the physics process, PDF, gluon radiation, and parton fragmentation. ${ }^{3}$. In our version we enable the decay of all non-stable particles ${ }^{4}$.

The calorimeters are simulated as a grid in the coordinates $\eta$ and $\phi$ with a granularity similar to the CDF hadron calorimeter.

- The energy of each stable particle is deposited in a cell of the grid according to its coordinates $\eta$ and $\phi$.

- The information contained in the grid is used for jet reconstruction. Note that in this way all detector effects are turned off.

We are considering just one interaction per crossing and we do not account for effects caused by the magnetic field. The nominal di-jet invariant mass distributions have been obtained using the two leading jets with a transverse energy above $15 \mathrm{GeV}$ and within a pseudorapidity interval of \pm 2.0 . In addition to the reconstruction tools described above, an event display has been developed in order to study pathologies and tails. Fig. 5.6 is an example of such a display, where jets within a cluestering cone of radius 0.4 are shown.

The towers with energy from the $\mathrm{W}$ are blue, while the other towers are red.

\subsection{Out Of Cone Corrections}

In order to compare the di-jet invariant resolutions obtained with different jet algorithms it is convenient to rescale the di-jet mass peak to the known W mass. High statistics Pythia $W \rightarrow q \bar{q}$ samples have been produced for different cone size using the CDF standard cluster algorithm. As shown in fig. 5.7, the lower shift in the peak caused by the energy loss due to FSR results becomes more evident with smaller cone sizes. We fitted the peak region of each distribution with a gaussian. The mean value and width of the invariant mass are quoted in tab. 5.3 for a cone size of $0.4,0.7$ and 1.0.

The out-of-cone correction has been studied for each cone size. We first asked for a spatial correlation between the parton from the $\mathrm{W}$ and the reconstructed jets. We selected jets with $\mathrm{E}_{T}>15 \mathrm{GeV}$ and $\Delta \mathrm{R}$ (parton-jet) $<0.5$. The approach to determine out of cone corrections is as described in sec. 4.2.4. The mean $\mathrm{E}_{T}$ parton- $\mathrm{E}_{T}$ difference is parametrized as a function

\footnotetext{
${ }^{3}$ For more details see sec. 1.5

${ }^{4}$ For stable particle we intend particles whose mean free path is bigger than detector size, for example pions or muons.
} 


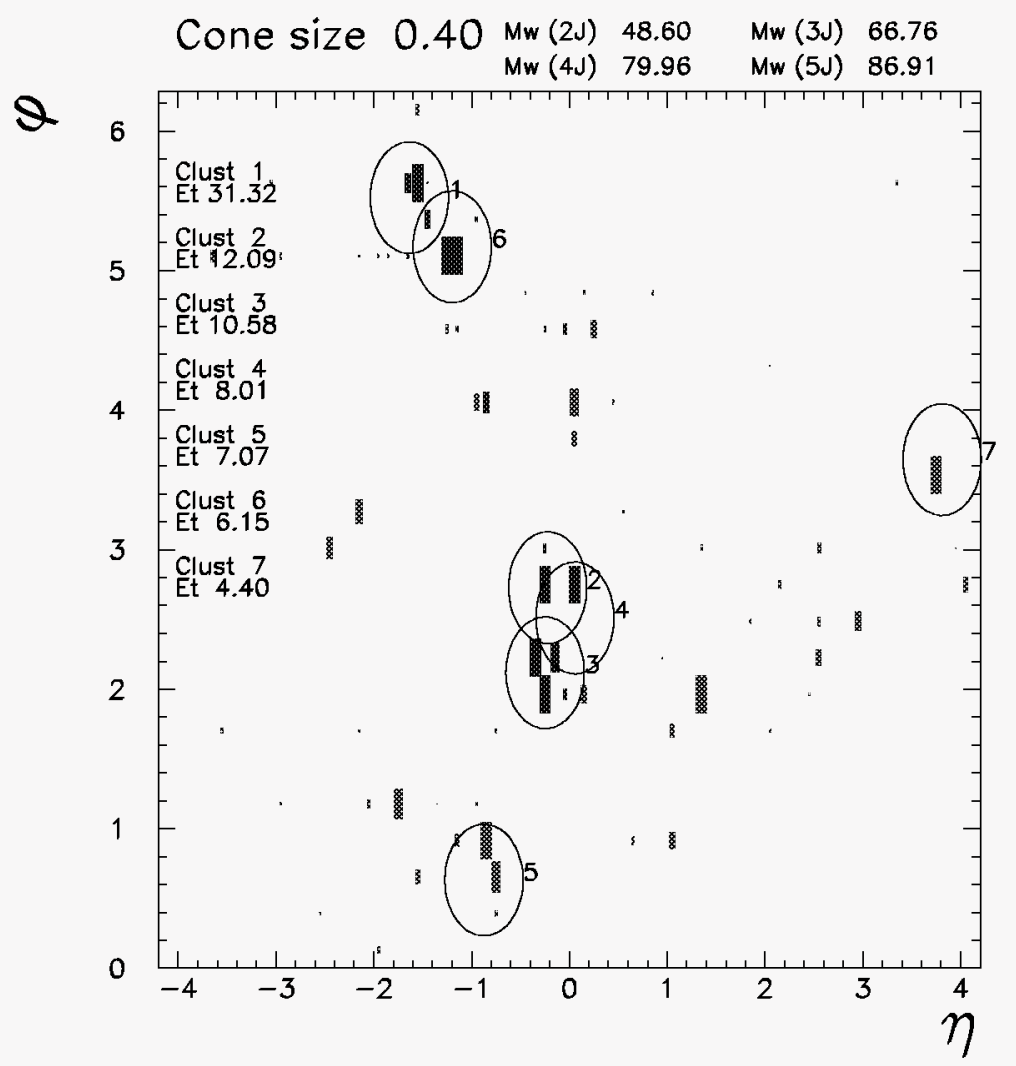

Figure 5.6: An example of the Paw-based event display.

of jet cone. This allows to corrects for the shift of the peak. However, in fig 5.8 we see that the tails grow with decreasing jet energy. As a first step, the shift of the peaks is used to determine the OOC corrections, while we will attempt to remove the tails in later sections. The offset corrections for the three cone sizes are reported in tab. 5.3.

We have compared the invariant mass distributions obtained after our additive corrections with those with a "JTC96 correction", and also with just a multiplicative correction applied to the mass. Our correction gives the

\begin{tabular}{|c|c|c|c|}
\hline Cone size: & 0.4 & 0.7 & 1.0 \\
\hline \hline Number of entries & 105,187 & 116,860 & 122,592 \\
\hline Mass $(\mathrm{GeV})$ & 74.1 & 77.4 & 79.3 \\
$\sigma_{\text {peak }}(\mathrm{GeV})$ & $2.9 \pm 0.2$ & $2.8 \pm 0.1$ & $3.2 \pm 0.1$ \\
\hline
\end{tabular}

Table 5.1: Di-jet mass resolution for the three different cone sizes. 


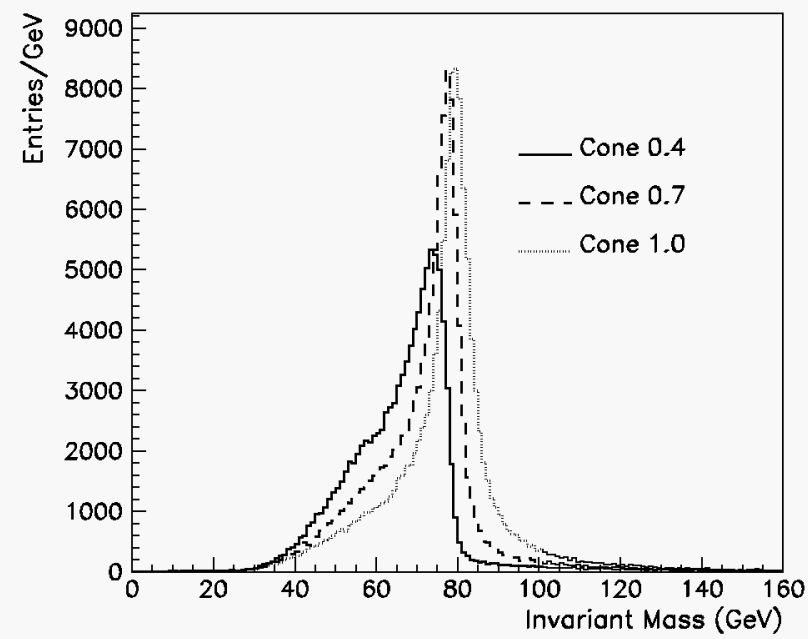

Figure 5.7: Di-jet invariant mass for cone radius 0.4 (solid), 0.7 (dashed), and 1.0 (dotted).
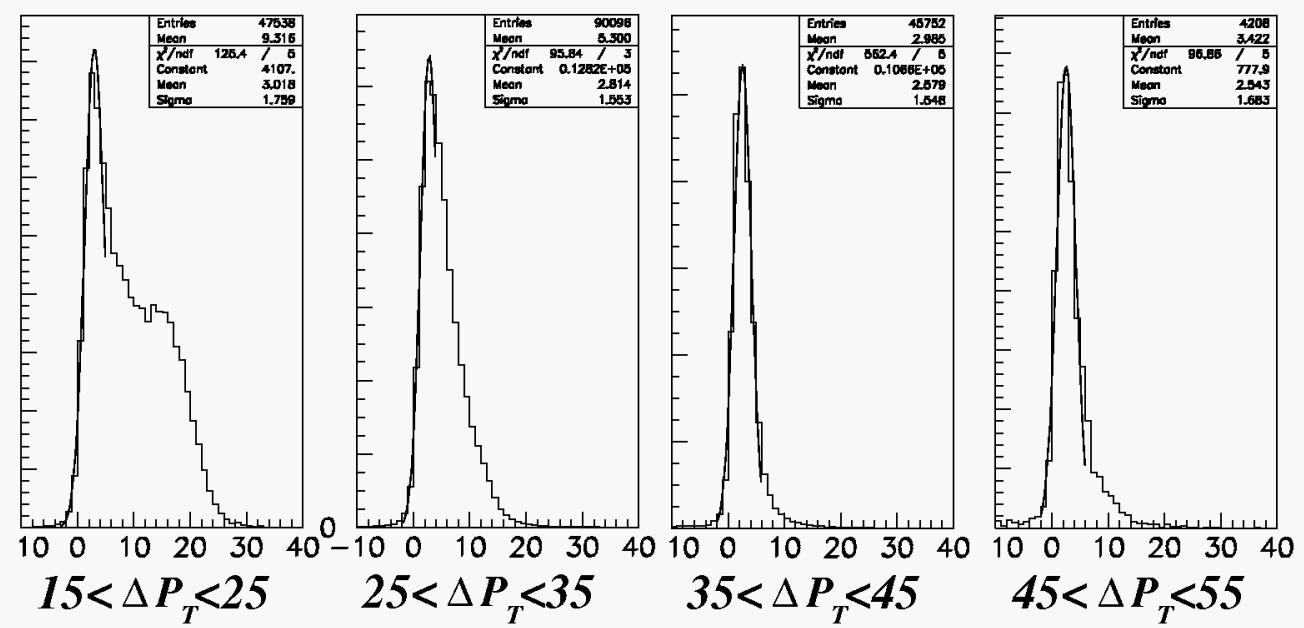

Figure 5.8: The distribution of the difference $E_{T}$ parton $-E_{T}$ jet for cone radius 0.4 and for four different slices of $E_{T}$ jet.

sharpest mass peak, as shown in fig. 5.9a). Based on this, and because of the simplicity of an additive correction, we believe that this kind of correction may well be the best prescription for this type of physics - even though it has 
never been used in CDF before. We show in fig. $5.9 \mathrm{~b}$ ) that the resulting mass peak for the three cone sizes agrees quite well with the nominal $\mathrm{W}$ mass.
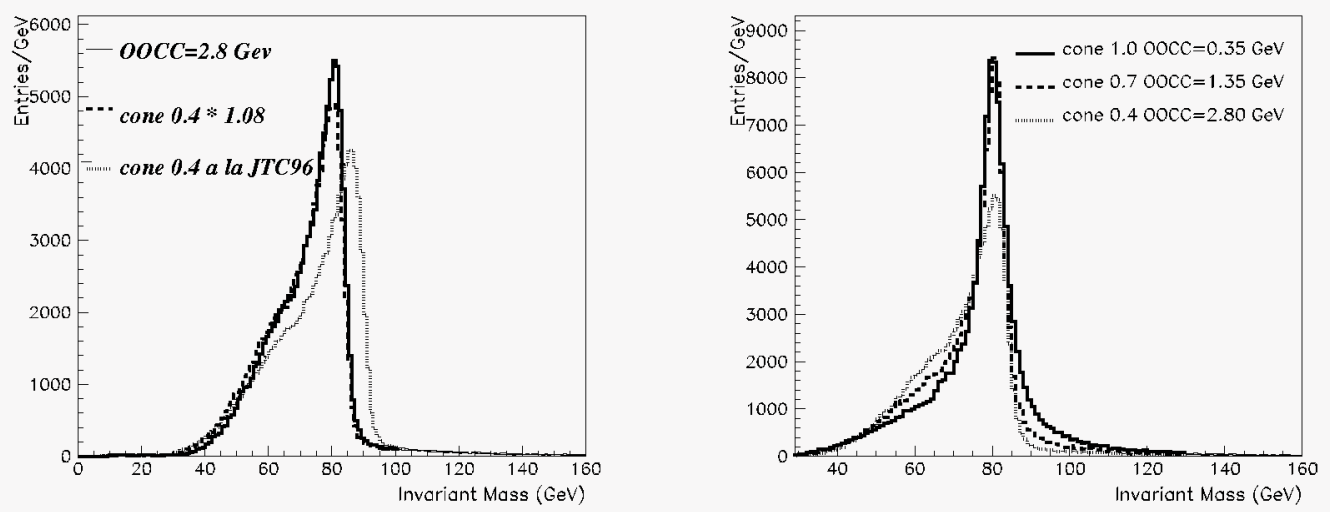

Figure 5.9: a) The invariant mass distribution with some out-of-cone corrections applied in the past by CDF. b) The invariant mass distribution for cone 0.4, 0.7, 1.0, after our out-of-cone corrections.

\begin{tabular}{|c|c|c|c|}
\hline Cone size: & 0.4 & 0.7 & 1.0 \\
\hline \hline OOC Offset $(\mathrm{GeV})$ & 2.8 & 1.35 & 0.35 \\
\hline
\end{tabular}

Table 5.2: Out-of-cone corrections for the three different cone sizes.

\section{$5.4 \quad K_{T}$ Algorithm}

In this section we will describe a new jet clustering algorithm, called $K_{T}$ Algorithm which has been advocated to improve dijet mass resolution in the past. We will describe the $K_{T}$ algorithm in the next subsection, then do a direct comparison between it and the cone algorithm for all three cone sizes. Another jet algorithm (ABC algorithm) will be introduced later.

\subsubsection{The $K_{T}$ Algorithm}

A detailed description of the $K_{T}$ algorithm can be found in [39]. We briefly summarize its main steps.

1. Define a list of jets. This is just a list of the four-momenta of hit calorimeter cells. 
2. For each jet, $i$, define:

$$
d_{i B}=p_{t i}^{2}
$$

and for each pair of jets, $i, j$, define:

$$
\begin{gathered}
d_{i j}=\min \left(p_{t i}, p_{t j}\right)^{2} \frac{R_{i j}^{2}}{R_{c u t}^{2}} \\
R_{i j}^{2}=\left(\eta_{i}-\eta_{j}\right)^{2}+\left(\phi_{i}-\phi_{j}\right)^{2} .
\end{gathered}
$$

$p_{t i}, \eta_{i}$ and $\phi_{i}$ are, respectively the transverse momentum, rapidity and azimuth of jet $i . R_{c u t}$ is a parameter somehow related to the cluster dimension.

3. Find the smallest number of $\left\{d_{i j}, D_{i B}\right\}$, and call it $d_{n}$, where $\mathrm{n}$ is the number of jets remaining.

4. If $d_{n}=d_{i j}$, merge jets $i$ and $j$ to give a single jet with four momentum

$$
p_{(i j)}=p_{i} \oplus p_{j}
$$

where $\oplus$ is some operation defining the recombination criteria which can be chosen in different ways:

(a) The covariant "E scheme" (the four-momenta of the two jets are summed up)

$$
p_{(i j)}=p_{i}+p_{j}
$$

(b) The " $p_{t}$ weighted scheme"

$$
\begin{array}{r}
p_{t(i j)}=p_{t i}+p_{t j}, \\
\eta_{(i j)}=\left(p_{t i} \eta_{i}+p_{t j} \eta_{j}\right) / p_{t(i j)}, \\
\phi_{(i j)}=\left(p_{t i} \phi_{i}+p_{t j} \phi_{j}\right) / p_{t(i j)} .
\end{array}
$$

(c) The "monotonic $p_{t}^{2}$-weighted scheme" with

$$
R_{(i j) k}^{2}=\frac{p_{t i}^{2} R_{i k}^{2}+p_{t j}^{2} R_{j k}^{2}}{p_{t i}^{2}+p_{t j}^{2}}
$$

5. If $d_{n}=d_{i B}$ then merge jet $i$ with the "beam jets" and jet $i$ is removed from the list.

6. Decide if another iteration is needed. If yes go back to step 2, otherwise the algorithm can stop. Sometimes one makes use of another parameter $d c u t$ to decide whether to end iterations. In a later section we will see the effect of different $d c u t$ values. 


\subsubsection{Comparison of $\mathbf{K}_{T}$ and Cone Algorithms}

Using the $K_{T}$ algorithm described in the last subsection, we now compare the $K_{T}$ and Cone algorithms with the same effective cone size. Fig. 5.10a is this comparison for cone size 0.7, and fig. 5.10b is for cone size 1.0. Essentially the cone and $K_{T}$ algorithms give the same mass distributions in all cases.
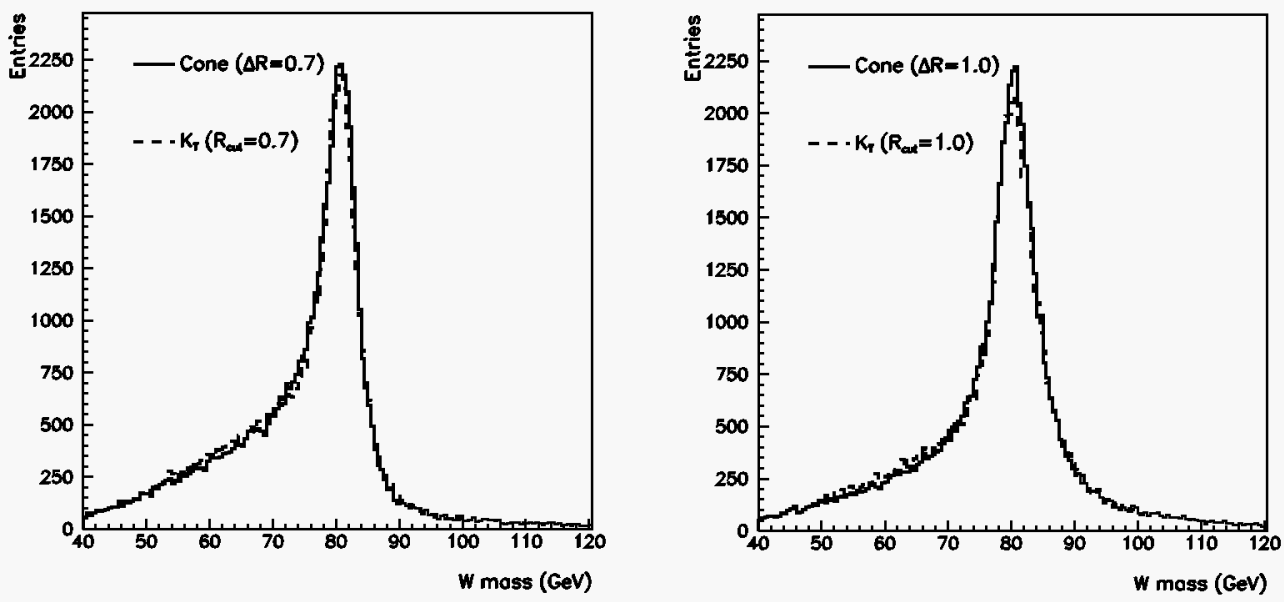

Figure 5.10: a)Comparison of $K_{T}$ and Cone algorithms with effective cone size 0.7 b) Comparison of $K_{T}$ and Cone algorithms with effective cone size 1.0.

To compare the algorithms more quantitatively, we have used four different measures of resolution. We use the width of a gaussian fit, the basic RMS of the distribution, and mass values including the 16 and $84 \%$ of the mass distribution $^{5}$. Fig. 5.11 shows all of these measures for the $K_{T}$ and cone algorithms. Generally the cone algorithm performs better than the $K_{T}$ algorithm. It also appears that generally the cones 0.7 and 1.0 perform better than the cone 0.4 clustering.

Choosing between the cone 0.7 and 1.0 algorithms is not really possible with these sensors since some are better with the $R=1.0$ and others are better with $\mathrm{R}=0.7$. In a later section we will present a likelihood analysis which will address the question, "Which is the best cone size?".

\subsubsection{Variation of $\mathbf{K}_{T}$ Algorithm Parameters}

As described earlier, in the $\mathrm{K}_{T}$ algorithm one has a number of choices on how to merge jets, as well as different ways to decide when to stop iterating. We

\footnotetext{
${ }^{5}$ We call these mass values the $16^{t h}$ and $84^{t h}$ percentiles. They measure the size of the mass distributions tails.
} 

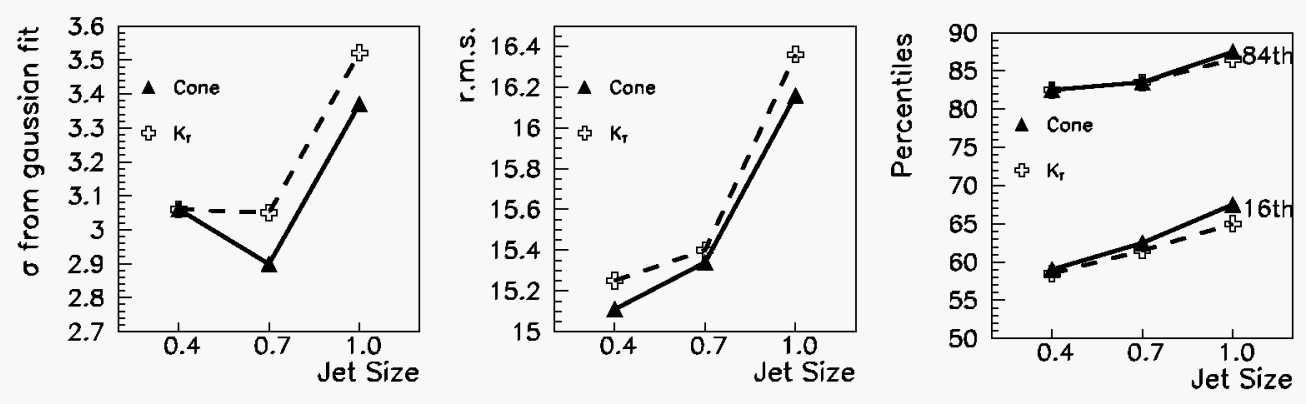

Figure 5.11: Comparison of various resolution parameters for the $K_{T}$ and Cone algorithms.

have compared these choices to see if another set of $K_{T}$ algorithm parameters would improve its performance significantly.
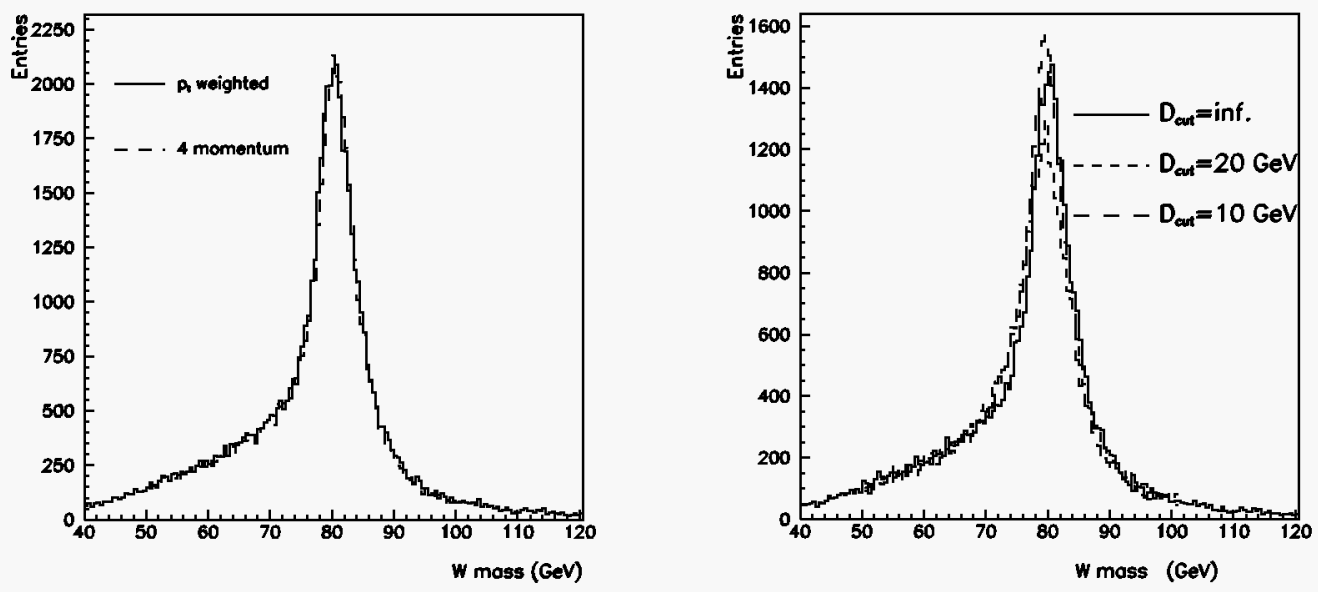

Figure 5.12: a) Comparison of different merging schemes within the $K_{T}$ algorithm. b) Comparison of different ways to stop iterating within the $K_{T}$ algorithm.

In fig. 5.12a) we compare the 4-vector merging with the pt-weighted merging and see no difference. In terms of deciding to stop iterating, we in the previous sections did not use a dcut parameter to stop iterations. In fig. 5.12b) we compare two different choices of $d c u t$ with our default choice of no cut $(d c u t=\infty)$. The changes in mass resolution are small, and our choice is intermediate between the other two choices. 


\subsection{Merging In Extra Jets With The Cone Algorithm}

Since the $K_{T}$ algorithm did not provide an improvement in the low mass tails due to FSR, we will return to the cone algorithm with cone size 0.4 . We will attempt to improve this tail by merging in extra jets and forming the multi-jet mass. We will only consider the five highest Pt jets, we found that there are almost no cases where a total of six or more jets are merged. To illustrate the potential usefulness of such merging, we show in fig. 5.13a) the 2-jet and 3-jet mass distributions, and also the multi-jet mass distribution when all the jets are known to have come from the W. Clearly there would be a big improvement if one could determine the correct jets. There are two main parameters in deciding whether to merge an extra jet: its energy and its distance from the main jet.
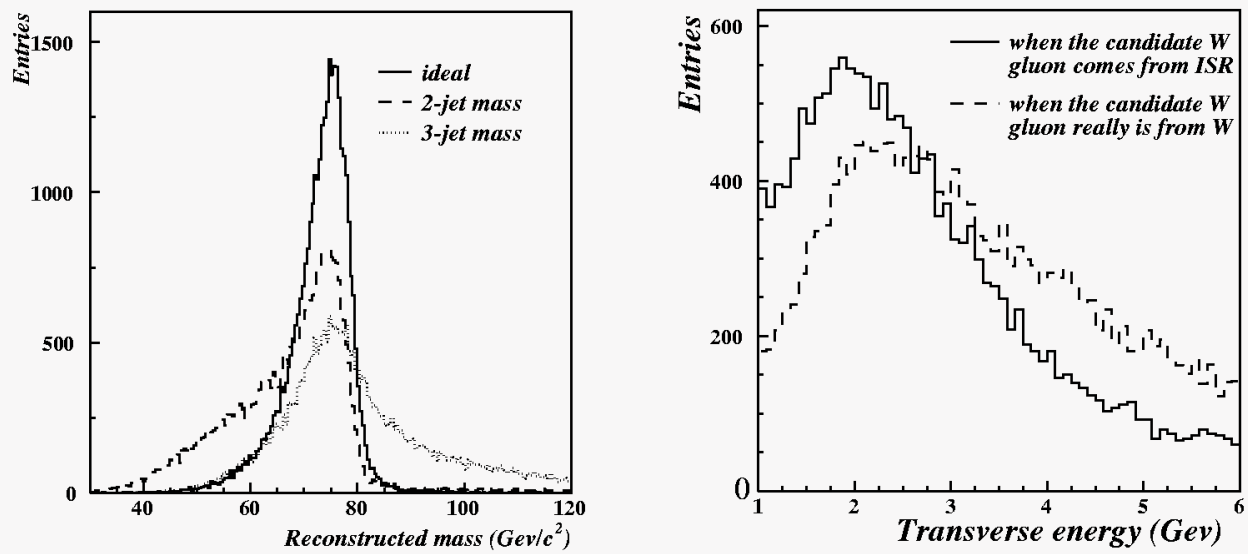

Figure 5.13: a) The 2-jet and 3-jet mass distributions, as well as the multi-jet distribution if all jets come from the $W$. b) Transverse energy of the jet closest to the second jet for ISR jets as well as for jets from the $W$.

In order to determine the energy cutoff for merging, we plot in fig. 5.13b) the Et of the jet closest to the second jet (requiring the second jet to come from the $W$ ), both when the extra jet is from the $W$ and when it is from ISR. The extra jet from the $W$ is usually harder than from ISR, with a distribution taking over at about at $3 \mathrm{GeV}$. In the following we shall only merge jets above $3 \mathrm{GeV}$.

In order to decide the distance cut for which jets should be merged, we have investigated the fraction of energy coming from the $\mathrm{W}$.

This is shown in fig. 5.14 for the leading three jets. Clearly the W energy fraction drops as one considers the less energetic jets. We then define two 

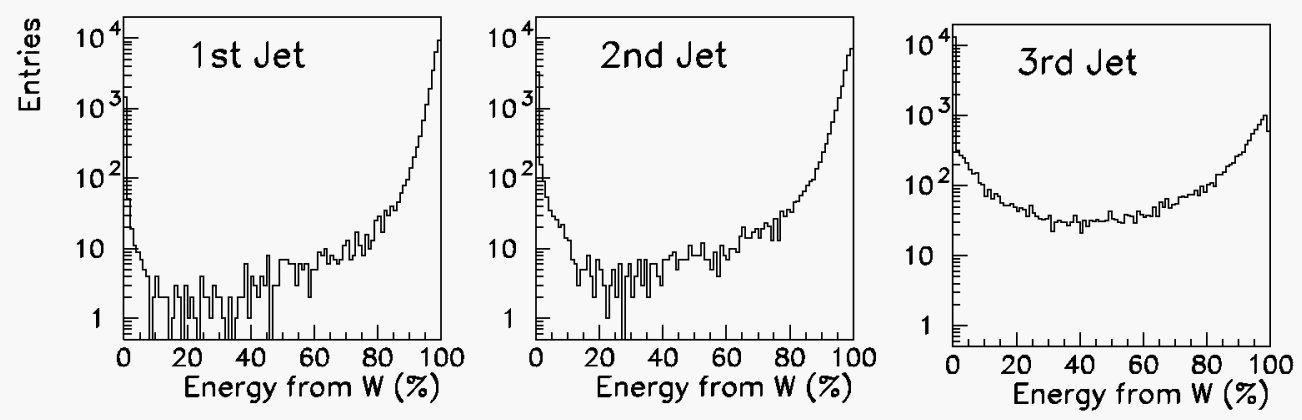

Figure 5.14: The fraction of energy from the $W$ for each of the first three jets.

classes of events based on the third jet $W$ energy fraction, those with $W$ energy fraction greater than $80 \%$, and those with $W$ energy fraction less than $20 \%$. The $\Delta R$ separation between the leading two jets and the third jet is shown in fig. 5.15 for these two classes, as well as for all events. Clearly a merging radius of 1.0 is optimal based on this plot.

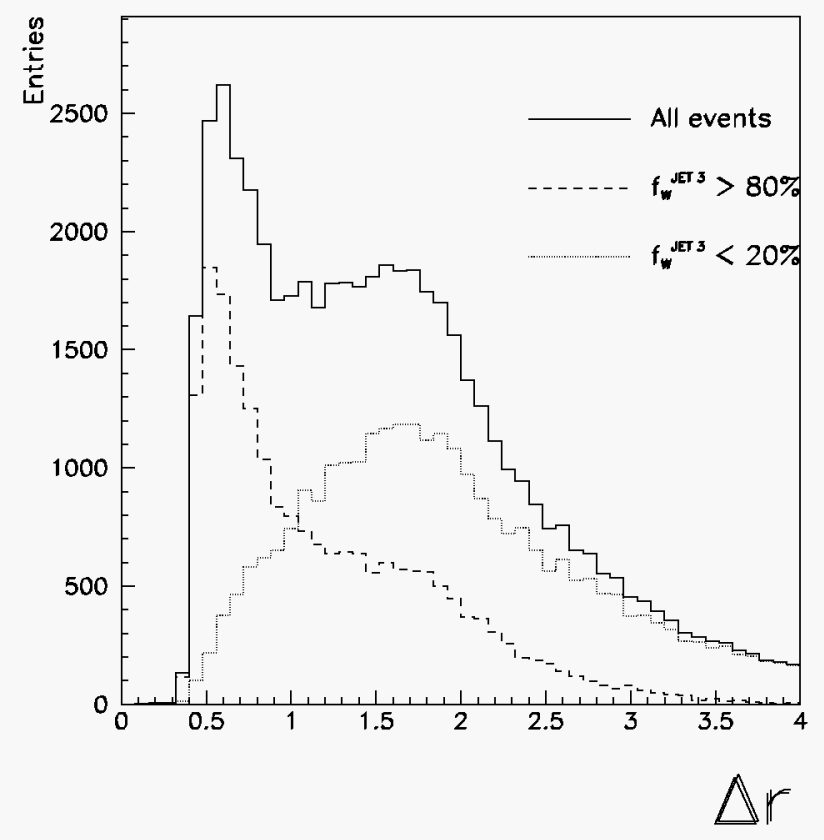

Figure 5.15: The $\Delta R$ separation between the third jet and the closest leading jet, for the two cases of a large $W$ energy fraction and a small $W$ energy fraction.

In fig. 5.16 we compare the basic cone 1.0 algorithm with the cone 0.4 algorithm, after applying a jet merging if jets are within $\Delta R<1.0$.

The distributions are almost identical. Notice also that the peak of the merged cone 0.4 algorithm is still at the $\mathrm{W}$ mass. This is further evidence that the new type of out-of-cone corrections described earlier is probably best 


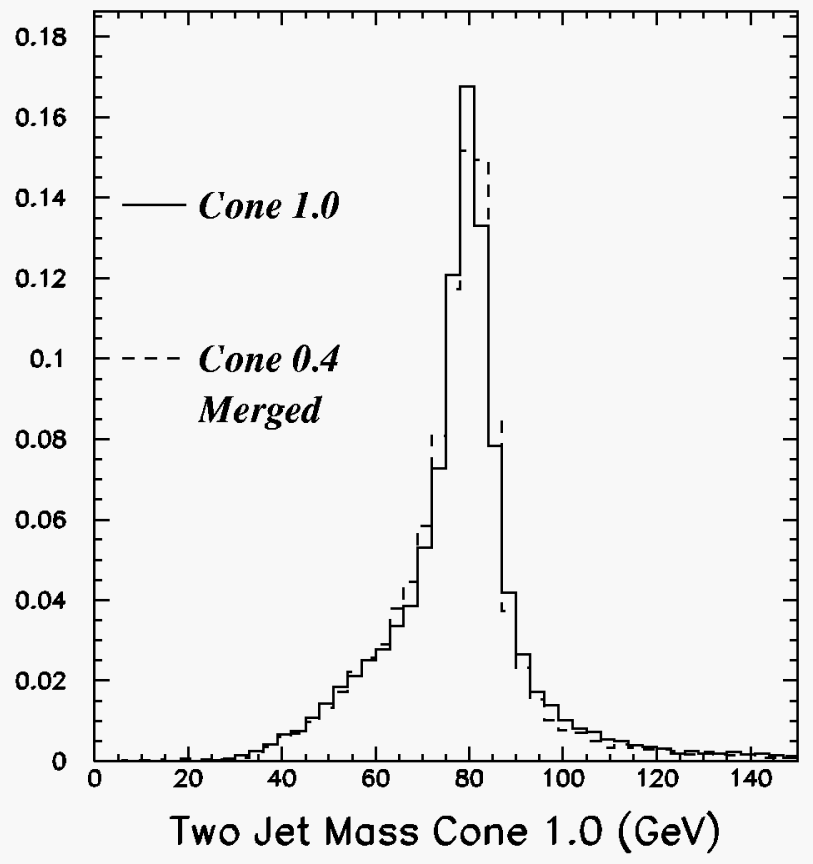

Figure 5.16: Comparison of the mass distributions for the cone 1.0 and the cone 0.4 algorithm (after merging extra jets within $\Delta R=1.0$ ).

for this kind of analysis.

Finally we would like to understand what effective cone size is really optimal. To do this we will construct a test-case situation with $400 \mathrm{~W}$ signal events, and 100.000 dijet backgrounds with $\mathrm{Pt}>10 \mathrm{GeV}$. We will then perform a likelihood analysis under two hypotheses: 1) background only, 2) signal+background. We will form the $\Delta$ Log-Likelihood for each case, and look for the best value. This is probably the most quantitative way to answer the question of which cone size is best. We will do this with the cone 0.4 sample, so the events will not change as we systematically change the merging radius of the extra jets.

This exercise is shown in fig. 5.17, for the four cases: 1) no merging, 2) merging within $\Delta R=0.7,3)$ merging within $\Delta R=1.0$, and 4) merging within $\Delta R=1.3$. The best $\Delta$ Log-Likelihood is with the merging within $\Delta R=1.0$, consistent with the $W$ energy fraction plot showed before. This figure shows there is a qualitative improvement in merging with either $R=1.0$ or $R=1.3$ over that with no merging or with merging within 0.7 , at least for this physics. The difference between using $R=1.0$ and $R=1.3$ merging is actually quite small. 


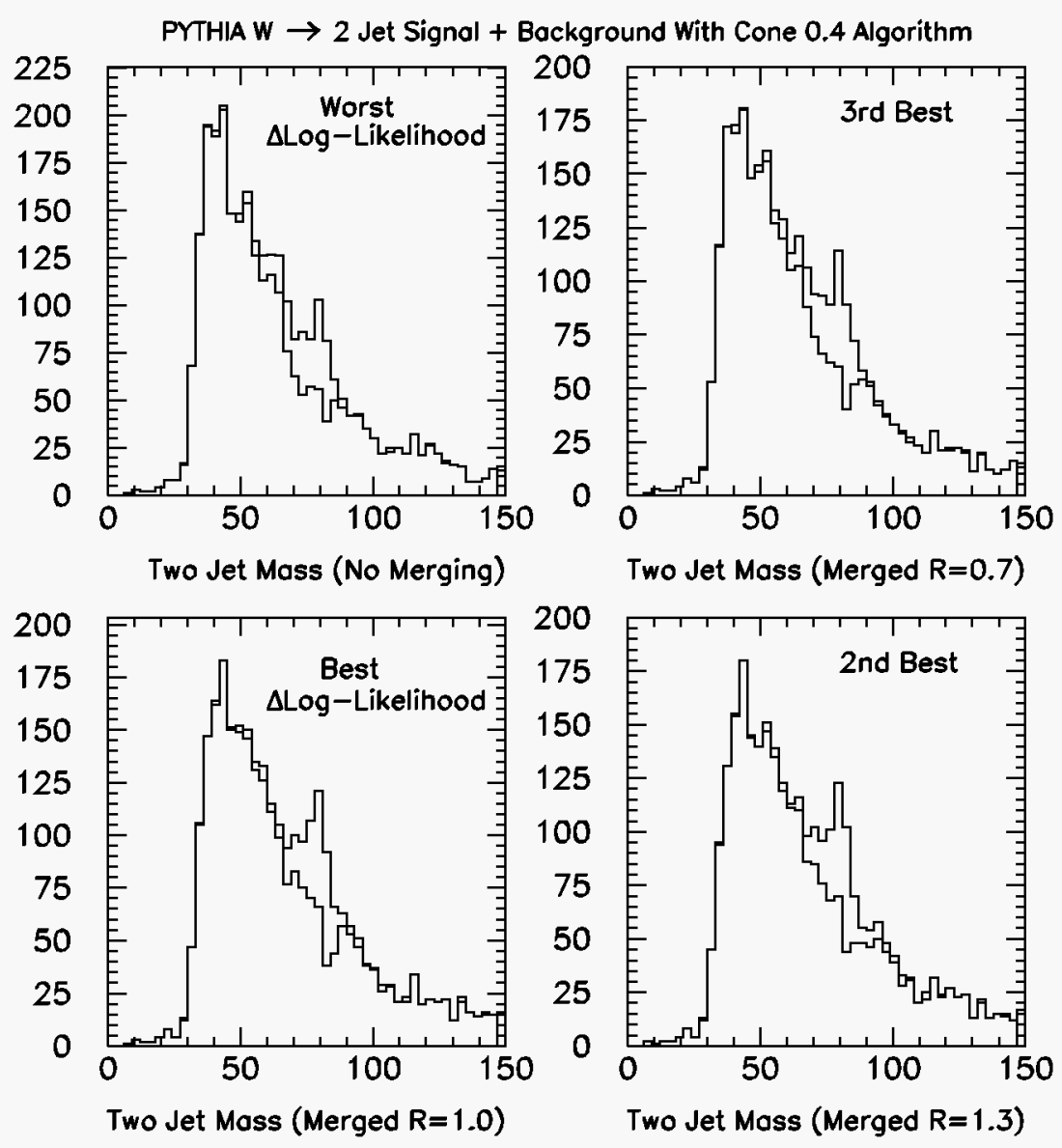

Figure 5.17: Test-case signal versus background example. In each case 400 signal events were used, and cone 0.4 clustering was performed. The merging of extra jets around the leading two jets is varied in the four plots. The best change in log-likelihood comes with merging jets with $R=1.0$.

\subsubsection{A More Sophisticated Method of Merging Jets}

As a further attempt to reduce the low mass tail due to final-state radiation, we investigated the option of using as a cut the fractional energy of the extra jet rather than a fixed $3 \mathrm{GeV}$ cutoff, and a variable merging radius.

The fractional energy is shown in fig. 5.18 for the extra jets from ISR and from the $W$. As shown earlier the $W$ typically produces more energetic jets. A better way to merge jets should be to expand the merging radius if the energy fraction is large. An algorithm to do this was developed, whose results are shown as the insert in fig. 5.19.

The resulting mass distribution is shown in the main part of this figure, and indeed it does give a slightly better resolution than the simpler merging 


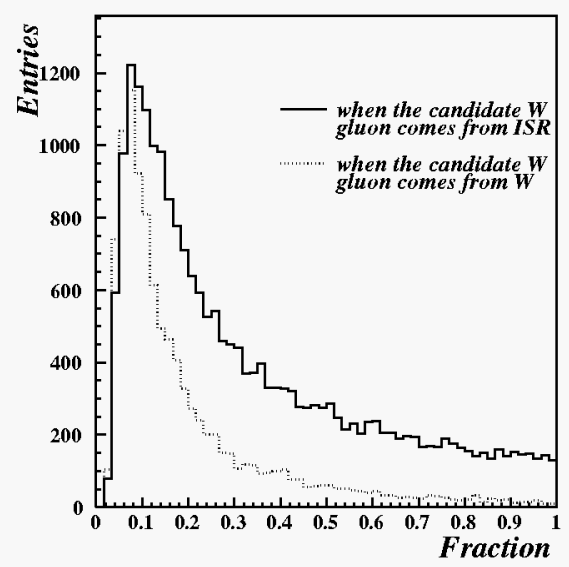

Figure 5.18: Jet energy fraction of the extra jet closest to the second jet for the case where the extra jet comes from the $W$, or from ISR.

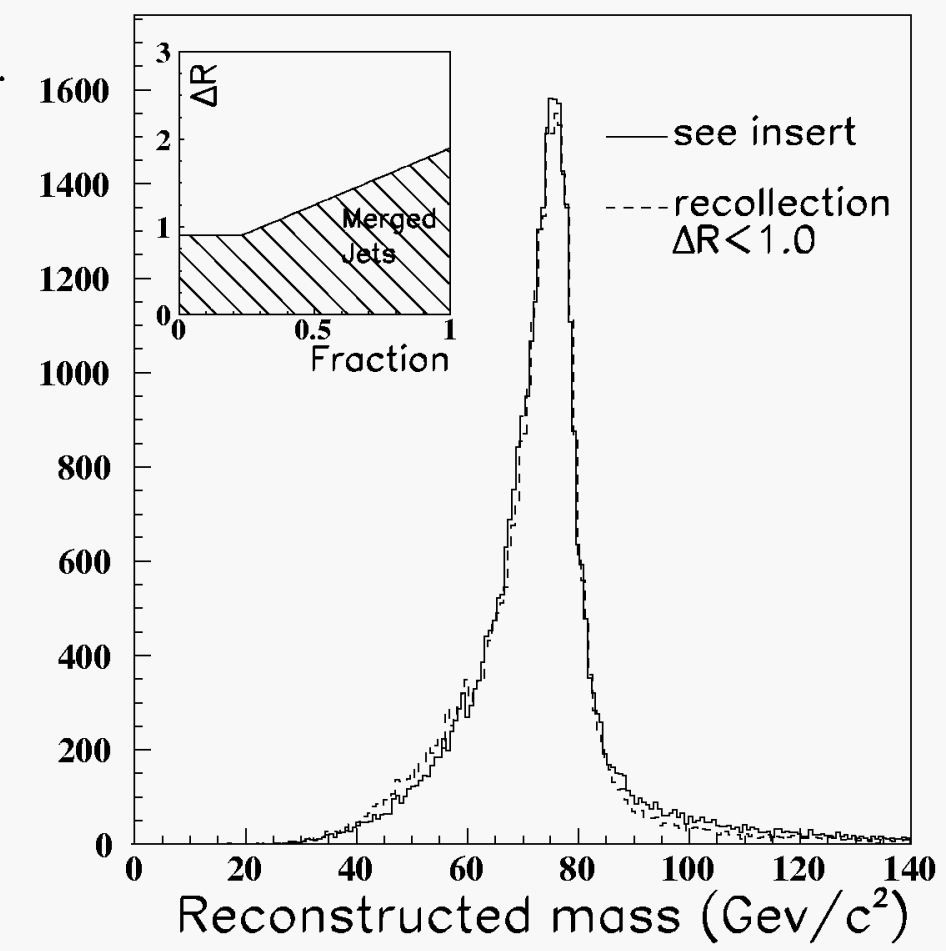

Figure 5.19: Sophisticated merging method described in the text (which uses a variable merging radius depending on energy fraction) compared to simple merging within $\Delta R=1.0$ described in the last section. 
of $\Delta \mathrm{R}=1.0$.

\subsection{The ABC Jet Algorithm}

There is an alternative to using a small cone and then merging-in jets within $\Delta R=1.0$ as done in the last section. One can use a large cone such as 1.0 or 1.3, but do a tower-by-tower estimate as to whether the tower is from the underlying event or the jet. If the tower is most likely to come from the underlying event, it is not added to the jet. We call this algorithm the ABC algorithm (Amorphous Big Cone).

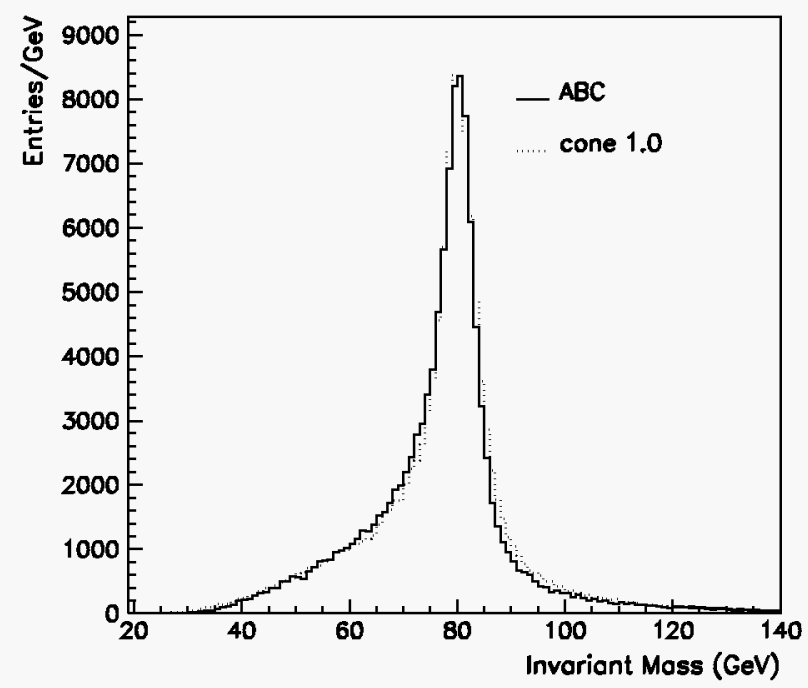

Figure 5.20: Invariant mass distribution from the $A B C$ algorithm (using a cone 1.0) compared to the basic CDF cone algorithm with radius 1.0 as well.

The heart of the algorithm is the right tower assignment criterion. Obviously low energy towers far away from the jet core are more likely to come from the underlying event. There are mainly two parameters to determine which towers are UE, the energy and the distance from the jet core. In order to determine the typical energy of a jet tower vs the UE, we ran a photon+jet Monte Carlo, and plotted the tower spectrum on the photon side, vs the jet side. The cross over point was at $1 \mathrm{GeV}$, with the jet side having the harder distribution. Since the last section showed that the basic size of the jet is $\mathrm{R}=1.0$, the simplest tower-determination algorithm we could think of was to reject towers if Et/R was less than $1 \mathrm{GeV}$. Thus in order for a tower near $\mathrm{R}=1.0$ to be included in a jet, it had to have $1 \mathrm{GeV}$ of Et. For a tower at $\mathrm{R}=0.5$, the tower only had to have $0.5 \mathrm{GeV}$ to be included in the jet. And so 
on. The invariant mass distribution of the $\mathrm{ABC}$ algorithm is compared to the basic cone algorithm, both with radii=1.0, in fig. 5.20. The $A B C$ algorithm has been corrected with a $1.6 \mathrm{GeV}$ offset to get the mass peak in the correct place, while the cone needed a $0.35 \mathrm{GeV}$ out-of-cone correction as discussed earlier. There is very little difference in the mass distribution. It is possible that a more sophisticated tower determination might help, but we have not pursued this further.

\subsection{Applying Extra Jet Cuts}

So far we have attempted to improve the low mass tail while keeping full efficiency for the signal. Now we will turn to the case where we have enough signal events to consider to apply a cut on the third jet, to further improve the signal to background ratio. We will use the same 400 signal events described above.

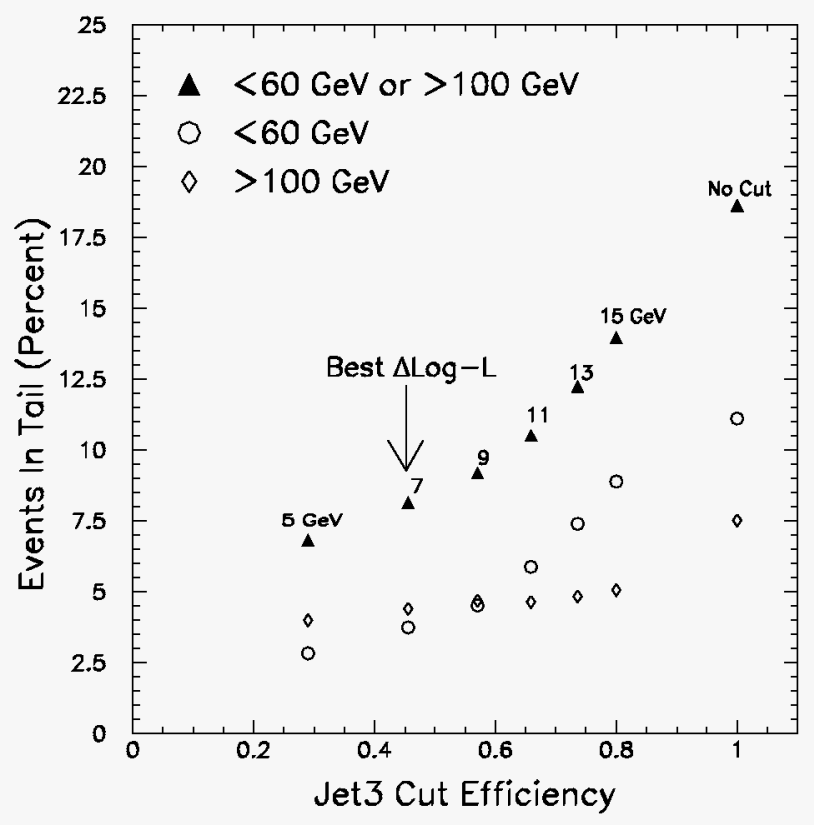

Figure 5.21: Percentage of events in the tails of the mass distribution versus the third jet cut efficiency, for several values of the third jet cut.

Fig. 5.21 shows the effect of applying third jet cuts. It shows the percentage of events in the tails of the mass distribution plotted versus the third jet cut efficiency, for several values of the third jet cut. The best $\Delta$ Loglikelihood between signal and background comes with a fairly tight cut of 7 $\mathrm{GeV}$, however more than more than half of the signal events are lost. This 
demonstrates the importance of finding ways of reducing the tails in these mass distributions.

\subsection{Conclusions}

Before going to the description of detector effects, let us summarize the results reached in the physics effects study:

- It is useful to separate sources of dijet mass resolution in physics effects and detector effects which can be studied separately.

- We have considered four different physics effects that contribute to dijet mass resolution. The dominant effect is final state gluon radiation, which causes a significant tail at low masses.

- The subject of out-of-cone corrections was been revisited, and we have derived the out-of-cone corrections based on the peak position, rather than on the mean, for the first time, motivating why this is better than "JTC96 correction" for this physics.

- An alternative jet clustering algorithm was compared with the standard CDF JETCLU algorithm, and they were found to be equivalent at least for this physics.

- The exhaustive study made to improve the low-mass tail showed a merging radius of 1.0 and a 3 rd jet cut of $7 \mathrm{GeV}$ to be optimal ones. 


\section{Chapter 6}

\section{Detector Resolution Study: Method}

In this chapter a new method to form the energy of a tower is presented. The track momentum measured by the Central Tracking Chamber will be used for the first time to define the tower energy. Towers will be divided in four classes and for each class a different method to determine the energy collected in the tower is adopted, that relies on which kind of particles hit the tower.

\subsection{Introduction}

The position of the core of the dijet mass distribution (see fig. 5.1) is shifted mostly by detector effects, such as calorimeter non-linearity, magnetic field effects, detector cracks and shower leakage. In the central calorimetry the response non-linearity is the largest contribution to the correction, due the non-compensating calorimeter $(e / h>1.0)$ and the calibration procedure. The response to high energy $(E>10 \mathrm{GeV})$ pions and electrons was measured in the test beam, which established the absolute calibration of the calorimeter modules. The EM compartment response is calibrated using electrons, and the HAD response is measured using pions which leave only minimum-ionizing signals in the EM compartment. Using this calibration prescription, and taking the energy in a cell as the sum of the energy of the EM and HAD compartments, the response to lower energy isolated tracks (mainly pions) has been studied in minimum bias events [40].

The average response is nonlinear with energy ${ }^{1}$, reproduced reasonably

\footnotetext{
${ }^{1}$ The nonlinearity of the calorimeter response needs some explanation. Even for a perfect detector (no cracks and infinite thickness), non-linearity is inherent in any calorimeter based on particle showering and having different responses to hadronic and electromag-
} 
well by the QFL and CDFSIM, the CDF detector simulation programs.

In jets, there are large fluctuations in both the neutral/charged mixture and the energy sharing between hadrons. Thus the non-linear calorimeter response causes a degradation of jet energy resolution and a bias, since the energy of jets with high charged multiplicity is underestimated. Starting from this consideration one can ask whether the information in the $\mathrm{CTC}^{2}$ can be usefully introduced to reduce the non-linearity effect in the measurement performed by the calorimeters.

\subsection{Preliminary Study Using CTC}

The standard jet-finding algorithm JETCLU (see sec 4.1.1) that sums the energy signalled by the electromagnetic and the hadronic calorimeter compartments, makes no use of tracking information. But for isolated charged hadrons, the CTC momentum measurement is much more precise than the calorimetric energy measurement for most energies of interest. Even without vertex constraints, the crossover for $\Delta p / p=0.002 p-i . e$. the resolution with which track momenta are measured by CTC - and $\Delta E / E=0.8 / \sqrt{E}-$ the calorimeter energy resolution for isolated charged particles - is about 50 $\mathrm{GeV}$. Even jets well above this energy which fragment into several charged hadrons might be better measured by the CTC.

It is interesting to investigate whether one may replace calorimetric information with tracking information for jets energy carried by charged particles. The main problem which may frustrate such an attempt is that the CTC is of no help at all for photons, neutrons and $K_{L} \mathrm{~s}$. Confusion between these particles and charged particles can seriously compromise the effort. Nonetheless for low energy jets, the energy may spread out sufficiently so that overlap of charged particle and photons may be less of a problem. Tracking system and calorimeter may provide complementary information.

For low energy jets, where calorimetric measurement gives large errors, tracking may give a significant improvement. For high energy jets, where track-finding may fail because of the increased track density in the jet core and where track-momentum measured becomes less accurate because of the higher track momenta, the calorimeter shower show its expected superiority.

To illustrate thoroughly the problems involved in using together calorimeter and tracking information we imagine three typical situations occurring in

netic cascades. A hadron induced shower has both an EM cascade (at the atomic level) and an hadronic cascade (at nuclear level). The hadronic component has a lower yield.

${ }^{2}$ The Central Tracking Chamber (CTC) is the sector of the calorimeter measuring $P_{T}$ for tracks located in the central region of polar angle $|\eta|<1.1$; for details see section 3.3 
an event:

(1) A $10 \mathrm{GeV} \pi^{+}$releases $2 \mathrm{GeV}$ in central electromagnetic calorimeter (CEM) and $6 \mathrm{GeV}$ in the Central Hadronic (CHA) calorimeter (for the moment we can imagine that the shower is confined in only one tower). Examining the picture of a $10 \mathrm{GeV}$ CTC track pointing to this tower one could correctly conclude that the $8 \mathrm{GeV}$ calorimeter info should be replaced by $10 \mathrm{GeV}$, thus improving the resolution.

(2) An $8 \mathrm{GeV} \pi^{+}$releases $2 \mathrm{GeV}$ in the CEM and $6 \mathrm{Gev}$ in the CHA. A $2 \mathrm{Gev}$ photon overlaps the $\pi^{+}$, and leaves an additional $2 \mathrm{GeV}$ in the CEM. Examining the picture one might conclude that the $10 \mathrm{GeV}$ of observed energy should be replaced by the $8 \mathrm{GeV}$ visible momentum, thus worsening the resolution. However the large EM fraction can be used to indicate the presence of a photon. Inspection of the Strip (CES) chamber (see 3.4) may help sort out the overlapping particles. The CTC and the calorimeter pictures would be made consistent, thus avoiding worsening the resolution.

(3) An $8 \mathrm{GeV} \pi^{+}$releases $2 \mathrm{GeV}$ in the $\mathrm{CEM}$ and $6 \mathrm{GeV}$ in the CHA. An overlapping $4 \mathrm{GeV}$ neutron leaves $4 \mathrm{GeV}$ in the $\mathrm{CHA}$. One would have to decide whether the high $E / p$ was due to poor energy resolution (and hence change $\mathrm{E}$ to $p$ ) or was caused by a neutral hadron. Combining the two informations would not be possible un an objective way in this case.

These simple examples already illustrate a lot of what can go wrong if a naive point of view is assumed - and we have already made the oversimplified hypothesis that a charged hadron releases its energy only in one tower. Before starting a long and very hard work to understand how to treat the overlap cases we have checked if one could expect it to be worth to introduce the tracking information in jet energy reconstruction. First of all, we have studied how many times charged and neutral particles are expected to fall in the same tower (like cases (2) and (3). With the particle level tool developed in the previous chapter, we found that in a typical $40 \mathrm{GeV}$ jet only $7 \%$ of towers in average are expected to hit by more then one particle. If we consider that in these events normally about 30 towers are energetic, we can estimate that the overlap cases are 2 or 3 towers per event.

As a second check we have estimated what would be the improvement on energy resolution if track momenta measurement for charged particles and calorimeter measurement of energy for neutral particles is used.

We bear in mind that if the improvement is not found be significant under this ideal condition no further work based on this approach would be 
justified. To check this, we have studied how jet energy resolution changes when we move from a condition of perfect detector - which measures exactly the energy of each particle - to the CDF detector with finite resolution, We monitor the resolution at every step.

The five phases we distinguish are:

(1) Perfect Detector. The energy of all particles falling into the cone is exactly measured. At this stage we miss those particles going out of cone.

(2) Cut on charged particles. Now we take into account the magnetic field effect. Charged particles with low $P_{T}(\lesssim 350 \mathrm{MeV})$ cannot be detected because they are bent by the magnetic field and cannot reach the calorimeter ("curl up effect").

(3) Nominal resolution for neutrals. In a real detector particle energy is known with finite resolution. At this step we take into account neutral particles by applying the nominal resolution. For photons we apply the CEM resolution, $13.5 \% / \sqrt{E}$. We assume that all neutrals are detected (no cracks effect). The hadron energy is still measured exactly.

(4) Nominal resolution for charged. We apply at this step the nominal resolution for charged particles as measured by central calorimeters (Electromagnetic plus Hadronic), i.e. $80 \% / \sqrt{E}$ in the hadronic compartment. No cracks effect is accounted for.

5 CDF detector All detector effects are included at this step applying the full simulation of the CDF detector, including the "JTC96 correction".

Table 6.2 summarizes the jet energy resolution obtained in the five cases. We have used a Monte Carlo sample of $\gamma+j$ et events with two different thresholds photon for $P_{T}$ and a cone radius $R=1.0$ in the jet search ${ }^{3}$. The jet energy resolution is taken as the width of the balancing distribution ${ }^{4}$ that is:

$$
\frac{P_{T}^{j e t}-P_{T}^{P h o t o n}}{P_{T}^{P h o t o n}}
$$

\footnotetext{
${ }^{3}$ For details see 4.1 .1

${ }^{4} \gamma+$ jet sample will be described in the next chapter. Now it is enough to know that in these events $P_{T}^{\text {parton }} \cong P_{T}^{P h o t o n}$ and so the Photon-Jet balancing can be seen as a direct Parton-Jet comparison.
} 
The main result is that the jet energy resolution can be improved by a better track momenta measurement. One also finds that it is dominated by the resolution of the hadronic calorimeter.

\begin{tabular}{|c|c|c|}
\hline \hline & $P_{T}^{\text {photon }}>15 \mathrm{GeV}$ & $P_{T}^{\text {photon }}>50 \mathrm{GeV}$ \\
\hline Perfect detector & $4.8 \%$ & $1.9 \%$ \\
Perfect but 350 MeV cut & $5.1 \%$ & $2.3 \%$ \\
Nominal for neutrals & $7.1 \%$ & $3.2 \%$ \\
Nominal for Tracks with 80\%/ $\sqrt{E}$ & $16 \%$ & $8.8 \%$ \\
detector resolution & $19 \%$ & $11 \%$ \\
\hline \hline
\end{tabular}

Table 6.1: Results of PYTHIA $\gamma+$ jet simulation. ISR turned off.

Some attempts to use CTC info was made in the past but the results were not very encouraging. We will mention two of them pointing out the method to compare them with the one adopted in this thesis and described in next section.

- In [43] the author divides the jet energy in "sub-clusters" and uses local information to get the best estimate of the energy for each subcluster. He completely replaces the CHA energy with the sum of the reconstructed track momenta and add this to CEM energy. Then an estimated amount of energy released by the charged hadron in the CEM is subtracted off.

This correction is a function of hadron momentum. Thus

$$
E_{\text {sub-cluster }} \longrightarrow\left(\sum_{\text {tracks }} p_{i}\right)+\left(E_{E M}\right)-\left\langle E E M_{\text {hadronic }}\right\rangle
$$

The final result is that an not appreciable improvement is reached, because of the large fluctuations in the energy released in the CEM by the hadron.

- Another attempt to use CTC info to make corrections to jet is made in $[44,45,46]$. In that study the aim of the authors was to correct the jet energy rather then introducing a new jet-finding algorithm. They focused their attention on the jets falling in crack regions ${ }^{5}$ and

\footnotetext{
${ }^{5}$ Cracks - or dead regions - are present in the calorimeter but not in the CTC detector. Charged particles lost in calorimeter cracks can be recovered using CTC track momenta measurement.
} 
corrected for the response non-linearity of the detector. Unlike the previous approach which corrected energy at a sub-cluster level, these corrections are applied on an event-by-event basis with the amount of correction depending on jet characteristics. A $7 \%$ improvement in jet energy resolution was found with this method.

\subsection{Classification Method}

It is clear that the gain achieved introducing the track measurement is milded by the energy fluctuations in the energy relased by hadrons in the Electromagnetic calorimeter.

If we want to use the track momenta and calorimeter response to estimate the particle energy, care must be taken to avoid double counting of contributions.

We have just showed how difficult it is to handle these fluctuations, both in the sub-clusters and the event-by-event approaches. The main idea developed in this thesis is to use, for the first time, the full granularity of the CDF detector performing corrections at tower level. In other words our goal is to use all available detector informations to determine as best as possible which kind of particle (charged or neutral) has released some or all of its energy in each tower.

After we will have done this, it will be easy to decide which detector section - CTC, calorimeter or both - to use to get the best energy estimate to be assigned to the tower.

For every event it is possible to identify a set of "golden" towers where one can use either tracking or EM calorimeter information only. Infect, we expect that when particles belonging to the jet are spread out enough, a large fraction of towers will be of "golden" kind. This is more likely to happen for low energy jets, where "JTC96 corrections" works worse.

With regard to "nasty" towers, as a last resource, we can adopt again the usual definition of energy tower, i.e. the calorimeter information only. But we expect that also for these towers tracks info can be used with benefit.

Postponing for the moment the problem of neutral hadrons, we want to "classify" each tower depending on the energy coming either from charged

particles or from photons. In the first case we flag the tower as "track tower", in the second one as "gamma tower". 


\section{"Track Tower"}

First of all we need to associate each track to a tower. We propagate the track to the central calorimeter radius - more precisely the strip chamber radius $R_{\text {ces }}=184 \mathrm{~cm}$ - where we evaluate the impact point coordinates $z$ and $\phi$. This propagation is a helical extrapolation to the center of the solenoid coil followed by a straight line extrapolation outside the solenoid to the strip chamber radius. The tower hit by track is called "target tower" and it is flagged as "track tower". A serious problem arises from the charged particle shower leakage. Showers are not, in general, completely confined in the target tower but extend also to the neighbour towers.

To study the isolated charged particle response CDF is used [41] to sum up the calorimeter energies in a $3 \times 3$ matrix centered around the target tower (" $3 \times 3$ window") and to compare this sum to the $P_{T}$ momentum of track measured by the CTC.

However, even if this choice proves to be the best one on minimum bias events where particles are very well isolated, it is too conservative to be used to classify towers in jet events.

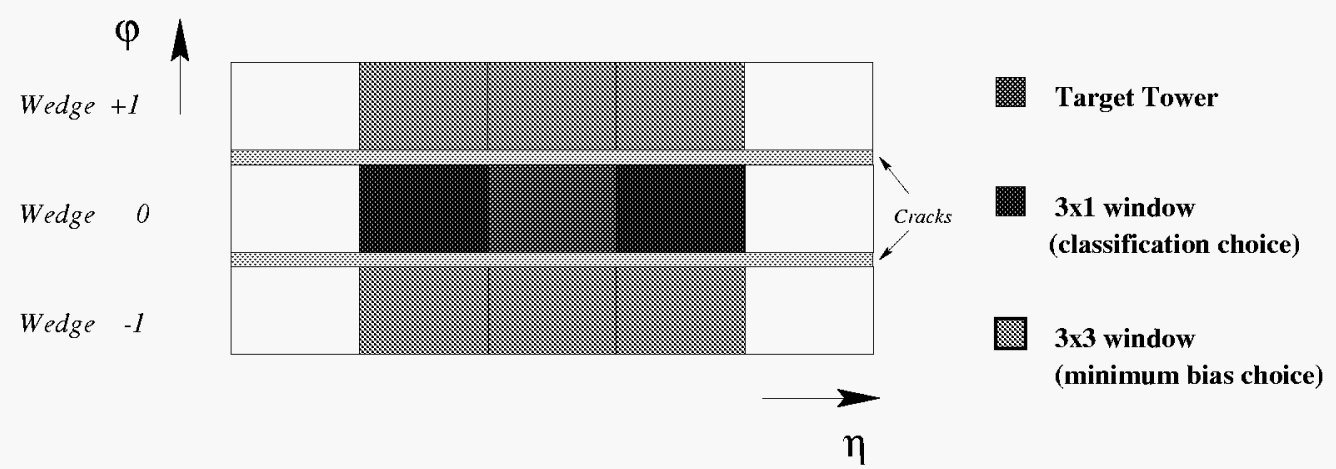

Figure 6.1: Schematic view of the calorimeter region near the impact point of a track. The red tower is the tower struck by the track ("target tower"). In minimum bias studies, a " $3 \times 3$ window" is associated to every track, the nine colored towers. For our study a " $3 \times 1$ window", the blue towers, was found to be enough.

We have looked at the energy release in towers placed in the two neighbour wedges $^{6}$ by the track shower (see fig. 6.1). Using a Monte Carlo sample of 10 $\mathrm{GeV}$ isolated tracks we have compared the calorimeter response summing the electromagnetic and hadronic energies of the 9 towers around the track tower and the 3 towers in the same wedge, (i.e. the target and the two neighbour towers).

\footnotetext{
${ }^{6}$ We remind that tower in a different wedge are physically separated by cracks, whereas towers separation inside the same wedge is obtained collecting the light coming from different cells in to different photomultipliers.
} 
The two distributions are quite similar - less then $1 \%$ different both in mean and width. Moreover in a jet of about $40 \mathrm{GeV}$ a large fraction of tracks have 2 or $3 \mathrm{GeV}$ as we can see in fig. 6.2 which can only have shower leakage in "target wedges".

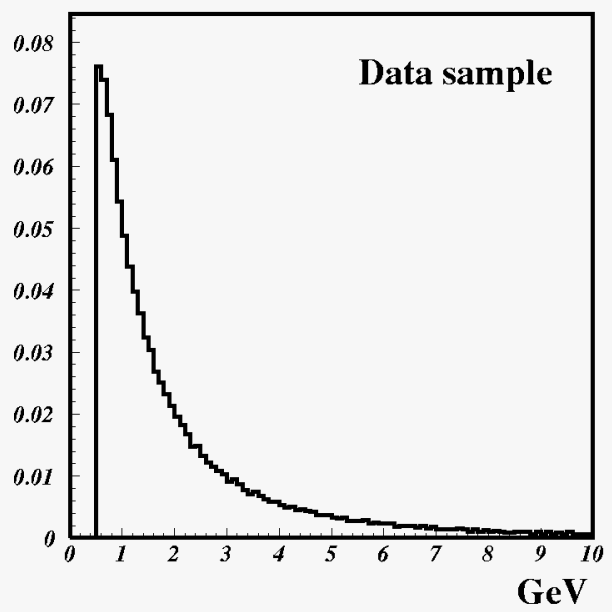

Figure 6.2: Tracks momentum distribution for $\gamma+$ jet data sample.

One could think to do a better job by using additional informations like the track position in the tower or the electromagnetic and hadronic energy fraction or combining them. We have made several attempts to look for interesting correlations using a Monte Carlo sample of isolated and monocromatic tracks $\left(P_{t}^{\text {track }} \sim 2 \mathrm{GeV}\right.$ ), but without achieving any result, mainly because of large fluctuations occurring at tower level.

Two of these attempts are reported in fig. 6.3. In the left plot, the energy in a " $3 \times 1$ window" and " $3 \times 3$ window" is compared to the track $\phi$ position in the tower. A little improvement in the leakage in close wedges can be noticed when tracks falls near tower boundaries, but it is clearly negligible.

The right plot shows the calorimeter energy collected in a $3 \times 1$ window versus $P_{T}$ track fraction when the shower goes beyond the wedge. Even in this case the correlation is negligible.

In conclusion, to classify a tower like "track tower" we require a reconstructed track pointing to the tower or to the two towers close in $\eta(3 \times 1$ window) as illustrated in fig. 6.1.

\section{"Gamma Tower"}

As previously mentioned, an indication of the presence of photons are electromagnetic clusters in the Strip Chamber (CES), a gas multiwire proportional 

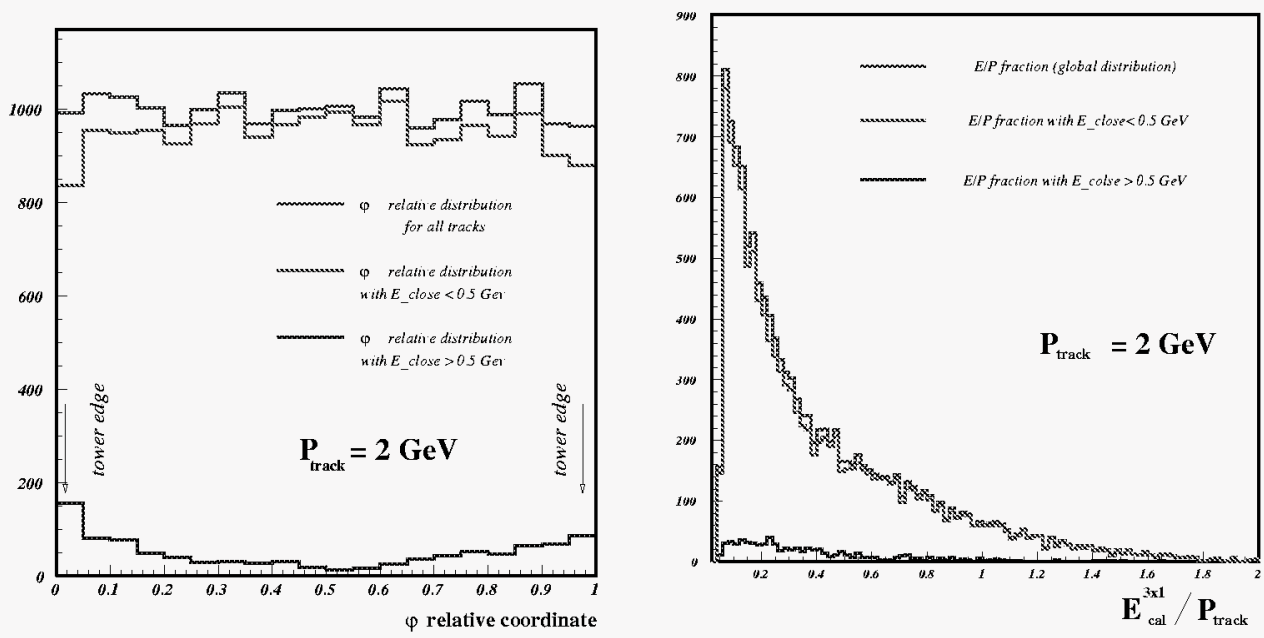

Figure 6.3: a) Relative $\phi$ position distribution of tracks inside target tower. The whole sample (red curves) has been divided in two subsamples depending on energy in close wedges (E_close) look for a possible correlation. The blue curve is obtained when the shower energy outside $3 \times 1$ window is large, the violet curve when this energy is negligible. b) Calorimeter energy (in $3 \times 1$ window) versus momentum fraction. In both distributions a Monte Carlo sample of monocromatic charged particles $\left(P_{T} \sim 2 \mathrm{GeV}\right)$ is used.

chamber embedded in the central calorimeter (characteristics of the CES system are described in [34]).

CES clusters, besides to merely indicate the presence of photons, allow to measure their position with good precision, being the resolution in these chambers about $2 \mathrm{~cm}$ along the $z$ coordinate.

However, some problems arise when CES information is used to detect photons. First of all, the minimum threshold energy required in order for a cluster to show up in the strip readout puts a limit to photon recognization. In other words, if a photon with less than $400 \mathrm{MeV}$ reaches the calorimeter, it is not able to form a sizable CES cluster and as consequence we cannot flag it as such ${ }^{7}$. Moreover, we have to take into account the cluster finding efficiency for showers above threshold that is not always one (it becomes one for photons above about $1 \mathrm{GeV}$ ).

Another item to care about is the shower leakage in neighbour towers, as in the charged particles case. The first thing to realize is that an electromagnetic shower does not in general propagate beyond the wedge boundaries.

\footnotetext{
${ }^{7}$ To lose photons with energy below $400 \mathrm{MeV}$ is not so dramatic. Charged particles below this threshold are lost because of the "curl up" effect as well and we have seen (see table 6.2) that this is not the main cause of degradation of jet energy resolution.
} 
This is because of the two $5 \mathrm{~mm}$ thick steel sheets on wedge boundaries. This restricts our study to the two closest towers $(3 \times 1$ window adopting the nickname used in the "track tower" section). The test beam experience, referenced in [47], teaches us that the electromagnetic shower is confined in only one tower (the target tower) if the particle hits the center of the tower. Using the full detector Monte Carlo simulation we have studied the electromagnetic calorimeter response to photons as a function of hit position in the tower.

\section{CEM response to photons}

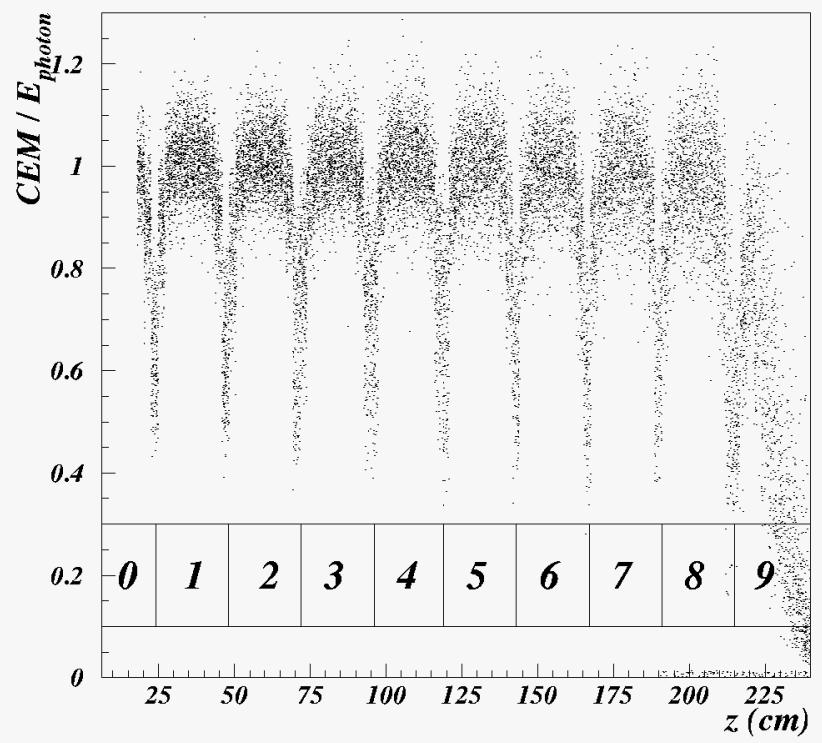

Figure 6.4: Calorimeter response to a gamma of $4 \mathrm{GeV}$ in the central region as a function of $z$ position in the wedge using the full detector Monte Carlo simulation. Towers boundaries is shown as well as the numbers of towers. We find a constant scale for gammas falling in the tower center (the electromagnetic calorimeter was calibrated using electrons). We can notice also what happens when a gamma falls near the edges, that is the efficiency go down. This is a proof that the shower spreads outside the target tower. Moreoever it is possible to see that the tower 0 (on left of plot near the $\eta=0$ crack) and tower 9 (at the end of the central calorimeter) have different behaviour.

Fig. 6.4 shows the calorimeter response as the CEM energy collected in the target tower divided by true photon energy to a single isolated photon of $4 \mathrm{GeV}$ for differents $z$ positions, that is equivalent to differents $\eta$ values of the photon. We can see that the response is almost one if the photon hits the 
center of tower (the same result achieved in the test beam), but there is a not negligible shower leakage in the nearest tower when the photon falls near the tower boundaries. Indeed, in fig. 6.5a) the same quantity is plotted but now as function of the $\eta$ position in the tower. It is clear that, if a photon falls near the tower boundary $\left(<20 \%\right.$ of the tower size $\left.{ }^{8}\right)$ some leakage is present in the close tower.

The prescription to flag a tower like "gamma" are then:

- A CES cluster is present in the tower, or

- A CES cluster is present in a neighbour (in $\eta$ ) tower but the distance in $z$ from the CES cluster and the edge of tower must be less than $20 \%$ of the whole tower length.
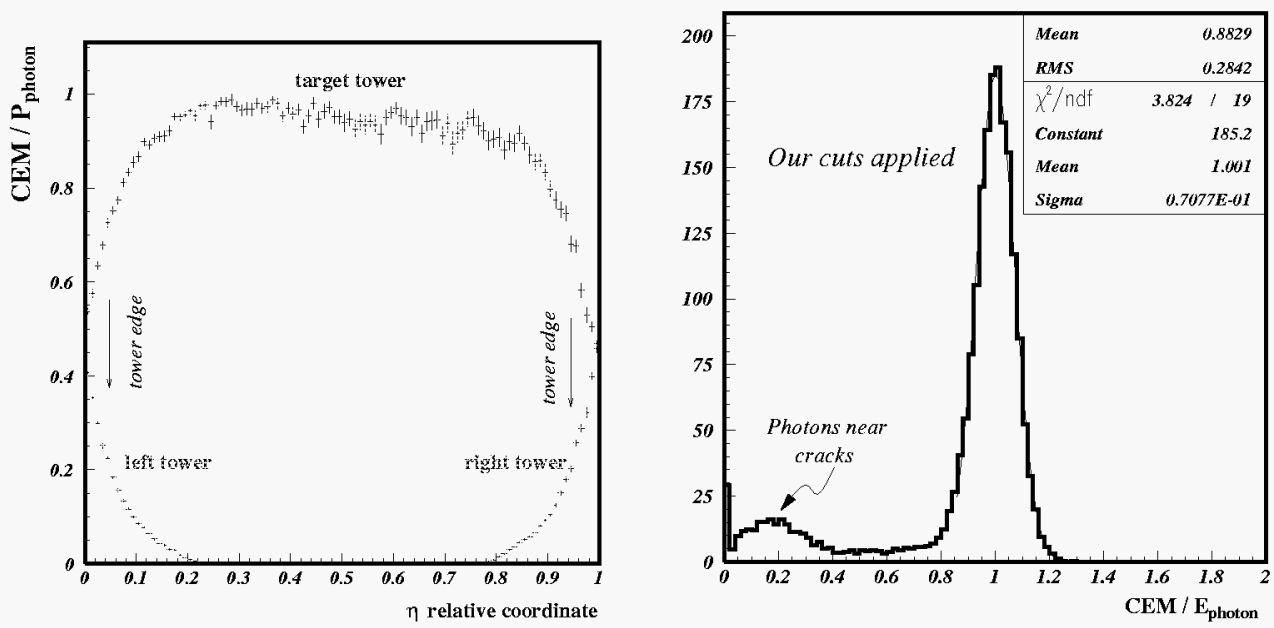

Figure 6.5:

a) CEM tower response as a function of hit point. b) CEM tower response as a function of photon energy. A Monte Carlo sample of isolated and monocromatic ( $\sim 4 \mathrm{GeV})$ photons are used.

Using the Monte Carlo $4 \mathrm{GeV}$ isolated photons sample we have studied the calorimeter response using these two prescriptions. The result is shown in fig. 6.5b). The energy resolution is in agreement with the nominal CEM resolution measured in the test beam ${ }^{9}$. In the figure one can notice also the

${ }^{8}$ The tower size is about $23 \mathrm{~cm}$.

${ }^{9}$ The CEM resolution is $13.5 \% / \sqrt{E} \mathrm{GeV}-\frac{1}{2}$. We have $E \sim 4 \mathrm{GeV}$, so we expect:

$$
\frac{0.13}{\sqrt{4}}=0.068
$$

to be compared with $\sigma \sim 0.07$ of fig. $6.5 \mathrm{~b}$ ). 
low detector efficency for photons near the $\phi$ cracks (events with $\frac{C E M}{\text { True }}<0.8$ ). We will take into account this effect later.

\section{"Mixture Tower"}

In the two previous sections we described and justified our choices in order to "classify" towers. Summarizing the method, we have:

- A tower is classified as track if:

- One or more tracks extrapolated to CES fall in this tower.

- One or more tracks extrapolated to CES fall in the two close (in $\eta)$ towers.

- A tower is classified as gamma if:

- A cluster CES is found in the tower or in the next towers close enough to boundaries.

Of course it is possible for a tower to satisfy both prescriptions and being classified both as "track" and as "gamma" tower. This is the overlap case described in the first section of this chapter. We called this kind of towers "mixture (or mix) towers".

Energy collected in a mixture tower comes from a track and from a photon. In this case we have to devise a method to estimate how much energy is released by the tracks to subtract off their contribution. This is exactly the same problem met by who, in the past, tried to include CTC informations in jet energy reconstruction. But now we have limited the overlap problem to a subsample of towers, where we have a simultaneous contribution from charged and from neutral particles. The remaining towers are the "golden" towers for which we can unambiguously use tracking or EM informations to improve the jet energy resolution.

The power of the method relies on how large the fraction is of "golden" towers with respect to the "mix" towers. Before to get this number we need to complete the method with the remaining things we have neglected up to now: the "not assigned" towers and "CES fakes clusters".

\section{"Not Assigned Tower"}

While a "mix" tower satisfies both "track" and "gamma" tower prescriptions there are some towers which satisfy no one. No tracks pointing to the tower and no CES clusters present. Analyzing some event displays of Monte 
Carlo events, where we know exactly what happens, we have found that "not assigned" tower is due to one of these three occurrences:

- A photon falls in this tower but no CES cluster is present. Either the photon is not energetic enough or the CES cluster finding algorithm has failed. In this case the hadronic compartment is not very energetic and the total CEM energy is below $1 \mathrm{GeV}$.

- A neutral hadronic particle $\left(n, \bar{n}, K_{L}\right)$ falls into the tower or in the neighbourhood. In this case both HAD and CEM calorimeters are energetic and the total energy collected can be above $1 \mathrm{GeV}$.

- One or more tracks fall in the closest wedge, in a $3 \times 3$ window around the tower. We have said that sometimes the hadronic shower can spread outside the wedge limit. Even if this occurrence is not very likely (for tracks with 1-2 $\mathrm{GeV}$ momentum) it is in principle possible and the presence of a "not assigned" tower is a confirmation.

Of course it is not too hard to recover at least the last case. We indeed decided to flag a "not assigned" tower near a track like "track tower" to avoid, in this way, problems of double-counting when reconstructing the jet energy. From now on the causes of "not assigned" towers are only the first two listed above.

\section{CES "Fake" Clusters}

In fig. 6.6 is plotted the $z$ distance between the track and CES cluster calculated at CES radius for "mix" towers having both photons and charged particles falling in. Being charged and neutral particles directions essentially not correlated at production, one might expect a triangular distribution for the difference $\Delta z=z_{\text {track }}-z_{C E S}$. However, this is not the case.

We have an excess of events around $\Delta z=0$. This means that sometimes a charged particle starts its shower before the Strip Chamber radius and this shower is detected making a CES cluster. In this case the CES cluster is not a test of the photon presence but is a fake that we have to remove not to classify mistakenly a "track" tower like a "mix" tower.

First of all from fig. 6.6 it is clear that "suspicious" CES clusters are very close to the track $(|\Delta z|<3 \mathrm{~cm})$. On the other hand, not all CES clusters near a track are "fake" clusters since $\Delta z=0$ is the most probable value in a triangular distribution. However, if CES cluster comes from a photon, one might guess a photon energy - and as consequence a CEM energy - above $0.8 \div 1 \mathrm{GeV}$. If instead the CES cluster is a "fake" one, the CEM energy 


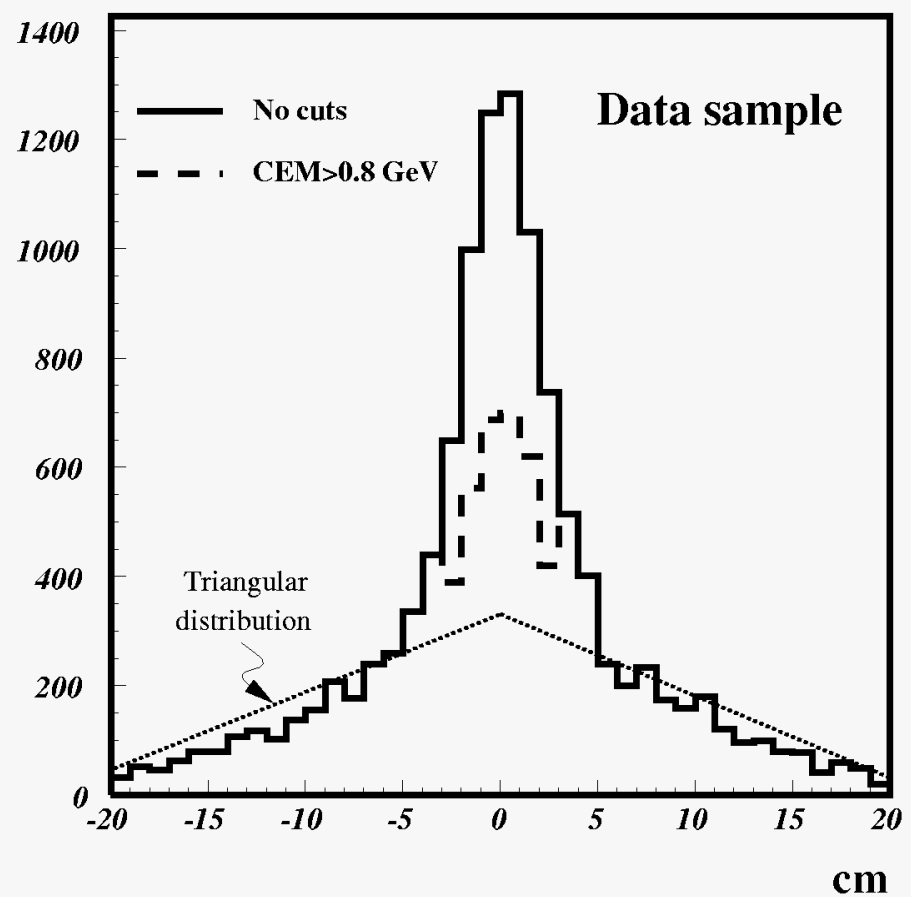

Figure 6.6: $\Delta z=z_{\text {track }}-z_{C E S}$ distribution when cut on CEM energy is or is not applied. The triangular distribution is showed as well. The "mix tower" in $\gamma+$ jet data sample is used.

comes only from the charged particle shower and it is not likely to carry a large energy. At last we decided to consider as "fake" clusters, those CES clusters with $|\Delta z|<3 \mathrm{~cm}$ and with a CEM energy less than $800 \mathrm{MeV}$. In fig. 6.6 is also plotted the $\Delta z$ distribution when our cut is applied. A distribution more similar to a triangular one is obtained.

\subsection{Classification Method: An Example}

After having described the new "classification" method, we'll see what happens when it is applied to a complete event. We have chosen a Monte Carlo event of the "Photon + Jet" sample. In this way we can know from the Monte Carlo list of particles exactly what particles fell in each tower and we are able to test the classification at the best.

In fig. 6.7 the "lego plot" and a side view of the calorimeter is shown. In the lego plot red towers show the electromagnetic energy and blue towers the hadronic one. The height is proportional to the collected energy. We notice a very tall red tower that corresponds to the Photon (of about $33.4 \mathrm{GeV}$ ) and almost opposite in azimuth a group of low energetic towers which compose 

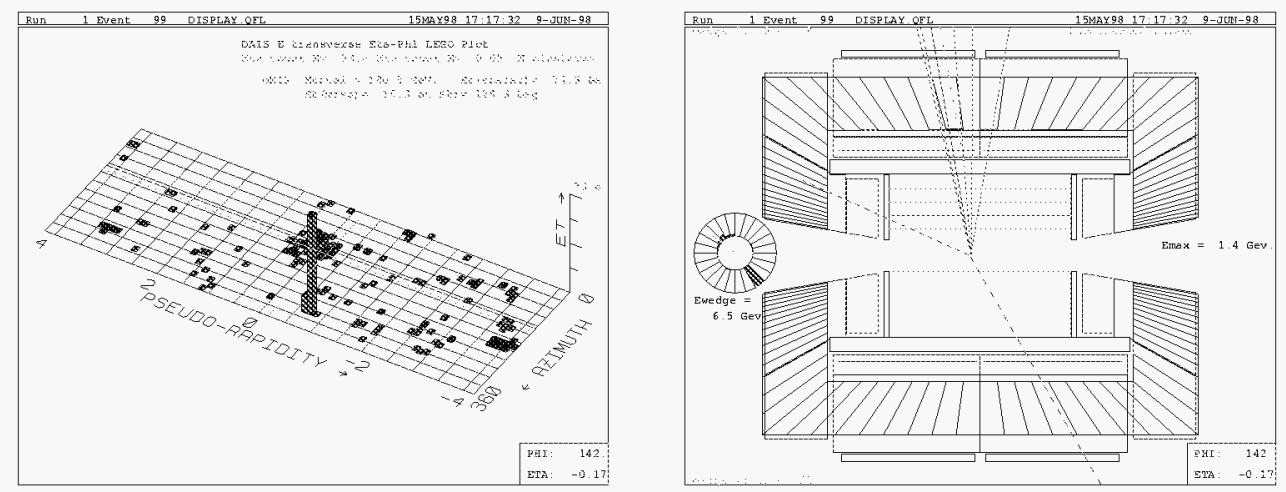

Figure 6.7: Display of an $\gamma+$ Jet event. a) Calorimeter transverse-energy deposition in $\eta-\phi$ space. The cylindrical calorimeter has been "unrolled" such that the axes of the grid represent the azimuthal angle around the beam line, and the pseudorapidity, definited as $-\ln \tan (\theta / 2)$, where $\theta$ is the polar angle with respect to the beam line. The height of each cell is proportional to its transverse energy $E_{T}=E \sin \theta$ ("Lego Plot"). b) View of the tracking chamber in the transverse plane ("side view"). The highest red tower corresponds to the photon. Almost opposite in azimuth is the jet.

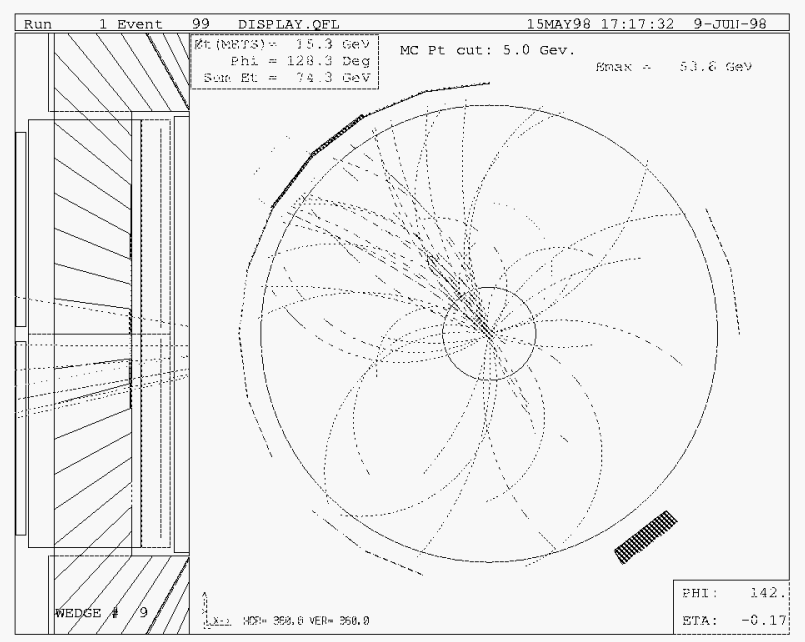

Figure 6.8: CTC view of the same event.

the jet. In the side view of the calorimeter we can see that Photon and jet are back to back in azimuth but not in $\eta$ (how one could expect). Fig. 6.8 is a "CTC view" of the event where the calorimeter release with the same color legend is shown as well. The isolated electromagnetic tower (red) is the Photon and, opposite in $\phi$, the jet. One can learn from the tracks bending that most charged particles have low energy (below $2 \mathrm{GeV}$ ).

Fig. 6.9 is again the lego plot but now only the central calorimeter is 

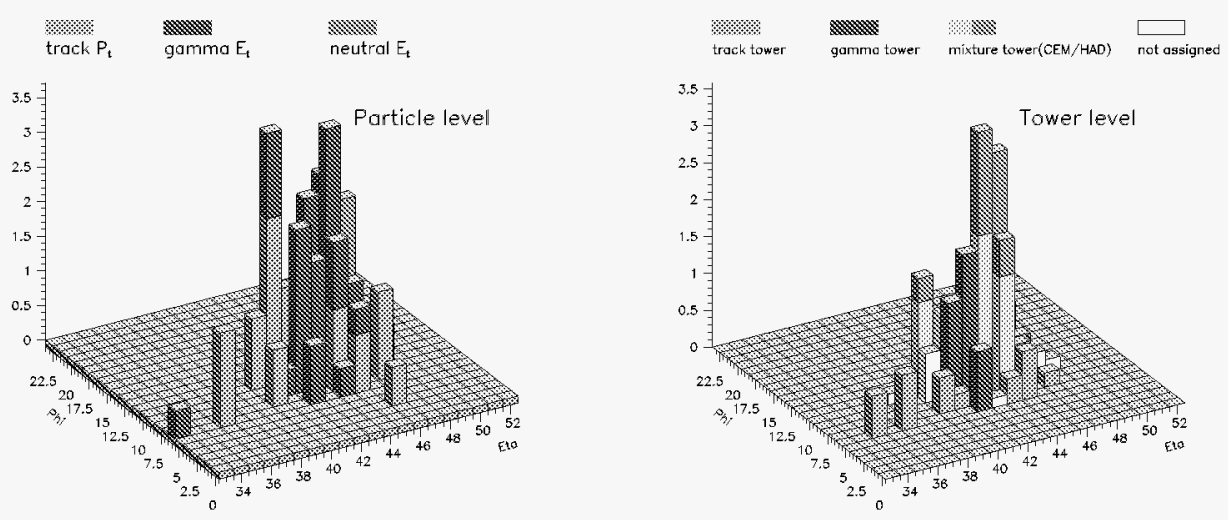

Figure 6.9: View of the central calorimeter of a Monte Carlo event. From the Monte Carlo list of particles we can check that, in average, every tower is hit by just one kind of particles. b) Same view of a real event after the classification method is applied.

shown. In fig. 6.9a) the particle scenario from Monte Carlo list is reported. with the Photon removed. A red tower means that a gamma fell in that tower, the blue means that a charged particle hit the tower and green is used when a neutral hadronic particle is present. The height is proportional to particle energy falling in the tower. If a tower has two colors, then two differents kind of particles hit it ${ }^{10}$.

In fig. 6.9b) we can compare the empirical situation after applying one classification scheme. The blue towers are classified as "track towers", the red ones are "gamma towers", the white ones are "not assigned towers" and the yellow/green (yellow for CEM energy and green for HAD energy) ones are "mix towers". It is possible to see that, for particles spread outside the core, the classification works very well. In the core, being the particles very close to each other, we have several mix towers.

It is clear now that the profitability of the "classification" approach relies on how many "mix tower" there are in the event. With few "mix towers" and several "golden towers", one can hope for a significant improvement in the energy measurement.

\footnotetext{
${ }^{10}$ Even if only few towers are hit by two kind of particles, the fraction of "mixture tower" will in practice be higher. That happens because at particle level particles do not shower. In other words, for each particle we consider only a $1 \times 1$ window. We know that the real situation is different from this ideal case.
} 


\subsection{How Many "Golden" Towers Are There?}

Using the whole data sample of "Photon + Jet" events, we have made a statistic of classified tower using the method illustrated in previous sections. The table 6.5 summarizes the situation:

\begin{tabular}{|l|c|c|c|c|}
\hline \hline Type & Number cells & CEM fraction & HAD fraction & Total \\
\hline Charged & $58.6 \%$ & $22.6 \%$ & $43.1 \%$ & $31.0 \%$ \\
Gamma & $8.8 \%$ & $11.6 \%$ & $2.5 \%$ & $7.9 \%$ \\
Charged-Gamma & $17.8 \%$ & $62.6 \%$ & $50.8 \%$ & $57.8 \%$ \\
Not Assigned & $14.8 \%$ & $3.2 \%$ & $3.6 \%$ & $3.4 \%$ \\
\hline \hline
\end{tabular}

Table 6.2: Tower classification for "Photon + Jet" data sample: a cut on photon energy $\left(P_{t}^{\text {photon }} \geq 40 \mathrm{GeV}\right)$ is applied.

These numbers need to be commented.

- Charged towers. There are about $60 \%$ of such towers but they carry only $30 \%$ of the event energy. This difference comes from shower leakage outside target tower, with more than one tower associated at each track. The HAD energy is split half in "track towers" and half in "mix towers". This is consistent with $30 \%$ of energy located in these towers. Indeed the particles in a jet can be approximately restricted to $\pi^{+}, \pi^{-}$ and $\pi^{0}$. From isospin symmetry, charged $\pi$ 's carry about $2 / 3$ of total energy and so, being the energy in "track tower" only $30 \%$, this means that charged particles are half in these towers and half in "mix tower".

- Gamma towers. Also in this case it is possible to show that the $11 \%$ of total CEM energy in the "gamma towers" is the expected value. But it is more important to notice the very low value of HAD energy, which means that the classification works well for this class of towers.

- Mixture tower. An interesting thing to notice is the difference between the fraction of mix towers (about 17\%) and the fraction of total energy contained in these towers (about 57\%). This means that in few towers we have overlap problems but these are very energetic, i.e. they are located in the core of jet. From what we have seen in previous sections, this does not appear as unexpected.

- Not assigned towers. We can see that few towers are in this class (about

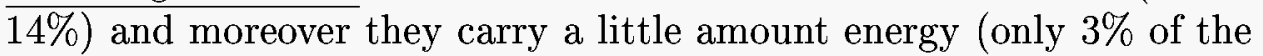


total). This is another confirmation of the validity of the "classification" approach.

\subsection{New definition of tower energy}

As a result of the classification process a label is assigned to each tower and the energy can be redefined as we are going to describe.

For "Track towers" we replace the calorimeter energy with the sum of the momentum of the tracks.

For "Gamma towers" we take just the CEM energy. We don't need to rescale the energy because Electromagnetic Calorimeter is calibrated using electromagnetic particles. Anyway we have to take into account the energy lost in the $\phi$-cracks. The deficit in response of the CEM calorimeter when a photon falls near the tower $\phi$ boundaries ${ }^{11}$ is shown in fig. 6.5. So the "gamma tower" energy is defined as $f_{g m} \cdot C E M$ with the factor $f_{g m}>1$. The value for $f_{g m}$ can be extracted from figure 6.5 as the inverse of the mean value of the histogram plotted on the right side i.e. $f_{g m} \cong 1 / 0.88 \cong 1.13$.

For "mix towers" we need a more sophisticated method to sum up CTC, CEM and HAD informations. Some double counting problems can arise as both photons and charged hadrons release their energy in the CEM compartment. We have to find a recipe to disentangle the CEM energy coming from electromagnetic particles and that from hadronic charged particles. A suitable solution is to subtract from CEM energy the expected contribution coming from charged particles detected by the CTC.

Thus the CEM energy released by charged particles in a given tower $\langle C E M\rangle_{c h}$ is expressed as:

$$
\langle C E M\rangle_{c h}=f_{\text {ch }}^{0} \cdot \sum_{\text {Target }} P_{t}^{c h}+f_{\text {ch }}^{+} \cdot \sum_{\text {Left }} P_{t}^{c h}+f_{\text {ch }}^{-} \cdot \sum_{\text {Right }} P_{t}^{c h}
$$

where the contribution from all the tracks impinging in the $3 \mathrm{x} 1$ window around the tower are taken into account. The values $f_{c h}^{0} \sim 0.2-0.3$ and $f_{c h}^{+}=f_{c h}^{-} \sim 0.05-0.08$ to be used on that expression are a result of studies on the response of the Calorimeters to Charged Particles performed by some CDF collaborators [41], [42]. As a cross-check for this expression we use it for towers flagged as "track tower". The value of $\langle C E M\rangle_{\text {ch }}$ can be compared with the measured CEM energy. Since, by construction, we have no photons in these towers the difference $\langle C E M\rangle_{c h}-C E M$ is zero within experimental errors as shown in figure 6.10

\footnotetext{
${ }^{11}$ Roughly speaking we can say that when a photon falls in the middle of a crack, its energy is lost.
} 


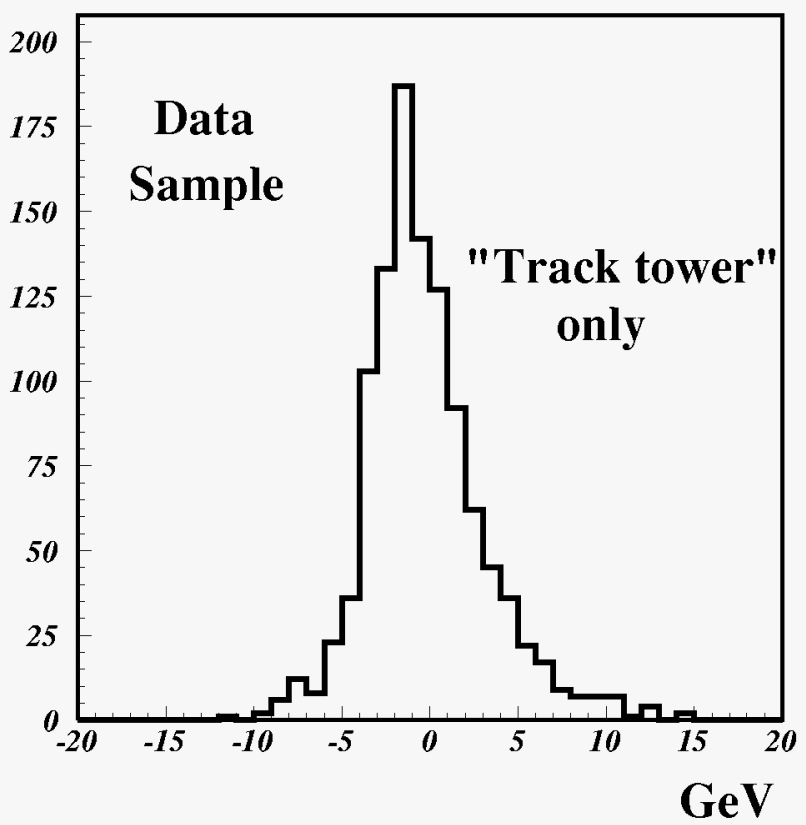

Figure 6.10: $\langle C E M\rangle_{c h}-C E M$ distribution for "track tower". This is a crosscheck showing that the energy released by charged hadrons in the electromagnetic calorimeter can be successfully parametrized with the sum of the momentum of all the tracks impinging in the $3 x 1$ window around a tower. The "track towers" in Photon + Jet data sample is used.

Thus the total energy carried by electromagnetic particles hitting a single tower can be expressed as:

$$
E_{P h o}=f_{g m}\left(C E M_{m i x}-\langle C E M\rangle_{c h}\right)
$$

Since "mix towers" are mostly in the core of jet, we would expect some neutral hadronic particles to be present. For this reason we decided to include a further correction term:

$$
E_{n e u t r}=f_{n e u t r} H A D_{m i x}
$$

with $f_{\text {neutr }} \approx 0.1^{12}$

\footnotetext{
jet.

${ }^{12} 10 \%$ is about the fraction of energy carried by neutral hadronic particles in a typical
} 
Finally the energy of a "mix tower" is defined as:

$$
\begin{aligned}
E_{M I X} & =E_{\text {ch }}+E_{\text {pho }}+E_{\text {neutr }}= \\
& =\sum_{\text {Target }} P_{T}^{c h}+f_{g m}\left(C E M_{m i x}-\langle C E M\rangle_{c h}\right)+f_{\text {neutr }} H A D_{m i x}= \\
& =\sum_{\text {Target }} P_{T}^{c h}+f_{g m}\left(C E M_{m i x}-f_{c h}^{0} \cdot \sum_{\text {Target }} P_{t}^{c h}+f_{c h}^{+} \cdot \sum_{\text {Left }} P_{t}^{c h}+f_{\text {ch }}^{-} \cdot \sum_{\text {Right }} P_{t}^{c h}\right)+ \\
& +f_{\text {neutr }} H A D_{\text {mix }}= \\
& =\left(1-f_{c h}^{0} f_{g m}\right) \sum_{\text {Target }} P_{T}^{c h}-f_{g m} f_{c h}^{-}\left(\sum_{\text {Right }} P_{t}^{c h}+\sum_{\text {Left }} P_{t}^{c h}\right)+f_{g m} C E M_{m i x}+ \\
& +f_{\text {neutr }} H A D_{\text {mix }}
\end{aligned}
$$

where the contribution from all the type of classified towers is included.

As can be seen we get a very long expression: this reflect the difficulty to deal with the "busy" mix towers.

To make this method work we need a smart choice of all the parameters in the above expression.

For "not assigned towers" we have to sum CEM and HAD energy because likely the energy released in these towers coming from neutral hadronic particles as mentioned above. This energy needs to be rescaled by a factor $f_{N A}>1$ to get the correct energy scale (this is a sort of absolute correction). This number has to be similar to the multiplicative factor needed to rescale the raw calorimeter energy to the photon scale extracted in fig. 6.11.

The factor used to rescale the distribution in figure $6.11(\sim 1.25$ from the fit mean) is used as $f_{N A}$.

Now for each kind of tower we are able to re-define its energy using a bigger set of detector informations which would result in an improved resolution as we will show in the next chapter. In table 6.6 the new tower energy definition is summarized. 


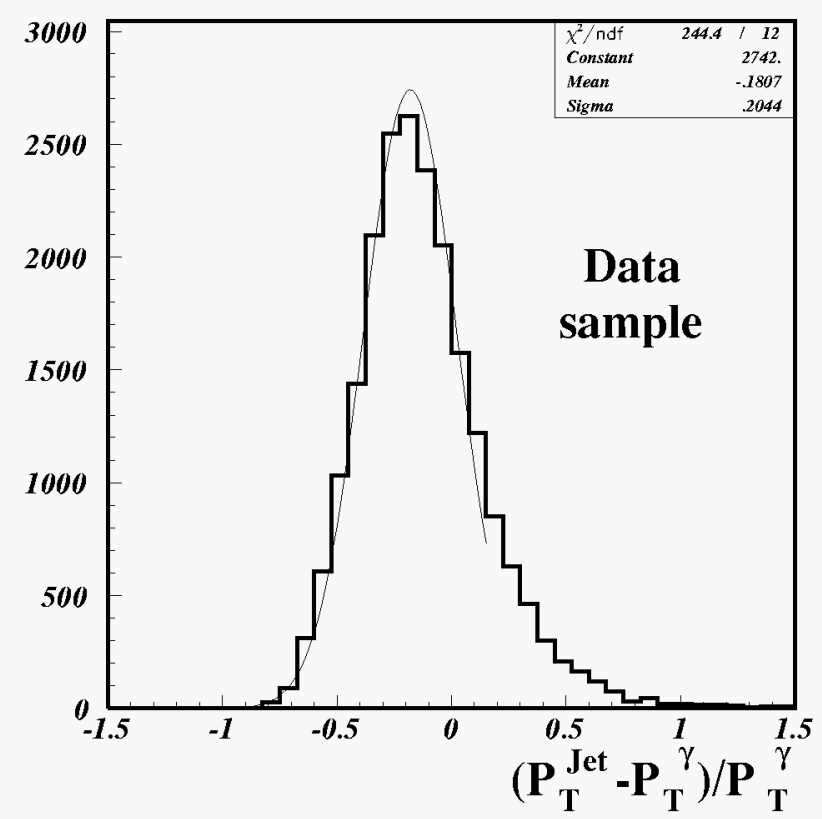

Figure 6.11: Photon-Jet balancing in real data using raw (CEM+HAD) energy for the jet. The non-linearity response of the calorimeter is the main source of the imbalance. From the mean fit it is possible to extract the correct factor correction factor.

\begin{tabular}{|c|c|l|}
\hline \hline Tower Type & Energy tower definition & Parameter values \\
\hline "Tracks" & $\sum_{\text {Target }} P_{T}^{c h}$ & No one \\
\hline "Gamma" & $f_{g m} \cdot C E M$ & $f_{g m}=1.13$ \\
\hline "mixture" & $\left(1-f_{c h}^{0} f_{g m}\right) \sum_{\text {Target }} P_{T}^{c h}$ & $f_{c h}^{0} \sim 0.2-0.3$ \\
& $-f_{g m} f_{c h}^{-}\left(\sum_{\text {Right }} P_{t}^{c h}+\sum_{L e f t} P_{t}^{c h}\right)$ & $f_{c h}^{-} \sim 0.05-0.08$ \\
& $f_{g m} C E M_{m i x}$ & $f_{g m}=1.13$ \\
& $f_{\text {neutr }} H A D_{m i x}$ & $f_{n e u t r}=0.1$ \\
\hline "not assigned" & $f_{N A} \cdot(C E M+H A D)$ & $f_{N A}=1.25$ \\
\hline \hline
\end{tabular}

Table 6.3: Summary of new tower energy definition 


\section{Chapter 7}

\section{Detector Resolution Study: Results}

In this chapter the comparison between the new jet energy reconstruction method and the standard CDF method to recontract jet energy is presented. First of all the "Photon + Jet" data sample and event selection are described. Then the jet momentum balancing is presented. A technique for extracting the calorimeter resolution follows. Finally, the detector resolution obtained with the new method is shown and compared with the one obtained with from the standard method.

\subsection{The "Photon + Jet" Sample}

The first thing that one needs when trying to improve the resolution is a method for monitoring the improvement itself. We decided to base our work on real data, in order to be unquestionably sensitive to detector effects that could be partly unknown or incorrectly reproduced in the simulation.

With this approach we do not know the true value of the measured quantity. We need to find a way to estimate the resolution from the data. Direct photon data sample could be appropriate for obtaining the jet $P_{T}$ scale relative to its initial parton $P_{T}$. The photon-like objects in this sample are well measured and the jets on the other side is expected to balance them in $P_{T}$. By checking how precisely this balance is obtained in the data we can test our ability to measure jets with the CDF detector. A direct comparison between the standard jet-finding algorithm ("JETCLU + JTC96" method) and the new one proposed in this thesis - "classification" method - can be usefully made with data using this kind of events. The leading order diagrams for photon production in $p \bar{p}$ collision are shown in fig. 7.1. The dominant diagram at the CDF energies is the first one of this set, gluon-quark Compton 
scattering.
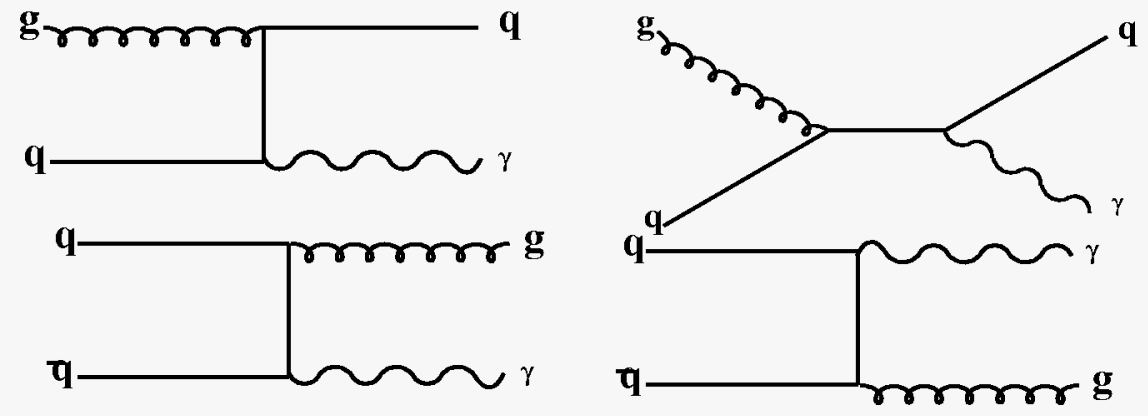

Figure 7.1: Leading order diagrams for photon production in $p \bar{p}$ collisions.

The first thing to realize about any direct photon study is that no set of cuts will be able to isolate a totally clean sample of photons. There is always a significant fraction $(0.3 \div 0.7)$ of dijet background that consists mostly of an isolated neutral meson decaying into photons $\left(\pi^{0}\right.$ 's, $\eta$ 's, ...). Therefore when in the plots we say "photon", we mean a mixture of direct photons and background isolated electromagnetic (CEM) clusters. We note also that several physical effects conspire confuse direct photon production. There are bremsstrahlung photons, bremsstralung gluons in the dijet background, $k_{t}$ smearing from initial state gluon radiation, clusters from the underlying event, and more ${ }^{1}$. In our analysis we reduce these effects by cuts on extra jets as will be described in a later section.

\subsection{Event Selection}

Usually, the term "prompt (or direct)" photons is used to indicate photons produced in the initial hadronic collision in contrast with those produced by decays of other particles like $\pi^{0}$ and $\eta$ mesons. The CDF detector is best equipped to measure prompt photons which are isolated (not accompanied by a large amount of nearby energy), and an explicit isolation cut is used in this measurement. The signal-to-background ratio is enhanced by the

1 " $\mathrm{Z}+1$ jet" sample, where the well measured $P_{t}$ of $\mathrm{Z}$ can be used to balance the jet, is a good alternative to "Photon +1 jet". Those events are free from dijet background due to the requirement of two leptons from $\mathrm{Z}$. However, the lower statistics of the " $\mathrm{Z}+$ 1 jet" sample compared to the "Photon +1 jet" sample limits an extensive use in the momentum balancing analysis. Therefore we have decided to study the more numerous "Photon + 1 jet" sample only. 
isolation cut. Since $\pi^{0}$ and $\eta$ mesons are produced in jets, requiring isolation greatly reduces (but does not eliminate) hadronics backgrounds whose production rate is many orders of magnitude large than $\gamma+$ Jet. Even applying a tight selection on photon candidates there is a large contribution from hadrons faking prompt photons. For our study we need a sample in which the "photon-objects" are isolated and balanced in $P_{T}$ by a recoiling jet, so the presence of this background does not affect our analysis anyhow.

Events passing any Level 3 inclusive photon trigger - with a requirement of photon $E_{T}>23 \mathrm{GeV}$ - are further filtered by requiring the photon to pass the selection criteria listed below:

(1) at least one strip or wire cluster in CES,

(2) CES $|x|<17.5 \mathrm{~cm}$ for the most energetic wire cluster,

(3) $14 \mathrm{~cm}<\mathrm{CES}|z|<217 \mathrm{~cm}$ for the leading strip cluster

(4) isolated $E_{T}$ in the towers within a cone 0.4 centered on the photon, $E_{T}^{\text {cone }}<4.0 \mathrm{GeV}$, excluding the photon cluster $E_{T}$

(5) No 3-D tracks pointing to the photon cluster,

(6) $E_{T}>25 \mathrm{GeV}$

(7) No second leading wire cluster with corrected $E_{C E S}>1.0 \mathrm{GeV}$ and CES $|\Delta x|>7.0 \mathrm{~cm}$ between the first and second leading wire clusters,

(8) No second leading strip cluster with corrected $E_{C E S}>1.0 \mathrm{GeV}$

(9) $\frac{Z_{\mathrm{T}}}{E_{T}^{\text {Photon }}}<0.8$

The candidate clusters were required to be accompanied by less than 4.0 $\mathrm{GeV}$ transverse energy in a cone of radius $\mathrm{R}=0.4$ around them (cut (4)). The $4.0 \mathrm{GeV}$ threshold represents the approximate underlying $E_{T}$ expected for direct photon events from the transverse energy distribution in minimum bias events obtained by placing at random a cone of the same size. In addition, these events were required to have usable strip chamber data (cut (1)). The shower had to be well contained in the strip chambers, where the whole shower profile is measured (cuts (2) and (3)). Events were discarded if they had a second strip chamber cluster with more than $1 \mathrm{GeV}$ in the same wedge photon hit (cuts (7) and (8)). This cut provides significant rejection against multiple photon backgrounds. The efficiency of this cut depends on the 
energy of the photon candidate and was well studied on test beam electrons. It ranges from 0.9 at $20 \mathrm{GeV}$ to 0.62 to $150 \mathrm{GeV}$.

Only events that had no reconstructed tracks in the central tracking chamber pointing at any calorimeter clusters were considered isolated photon candidates (cut (5)). Events were also discarded if there was a net imbalance of transverse $E_{T}$ (cut (9)). Events rejected by this cut were almost cosmic-ray events.

\subsection{Jet Momentum Balancing}

In this section, we describe the momentum balancing between the photon and the jet to compare the energy scale derived with the "JTC96" algorithm, to the one derived with the new "classification" algorithm.

\subsubsection{Data Sample Selection Cuts}

In sec. 7.2 the " $\gamma+$ Jet" event selection has been described. Further cuts were applied to study the performances of the new algorithm, as listed below:

- Central detector: The "classification" method has been developed only in the Central detector region. Only the central jets $\left(\left|\eta_{j e t}\right|<0.7\right)$ were considered. We have also required no energetic towers outside Central Detector.

- $\eta$-crack: No special corrections have been applied to the towers near the $\eta=0$ crack and where the response is not linear. For this reason, cuts on $\left|\eta_{P h o}\right|>0.1$ and $\left|\eta_{j e t}\right|>0.1$ have been applied.

- Extra jet activity: To select a good jet balancing the photon, we require one and only one jet with raw $E_{T}>8 \mathrm{GeV}$ to avoid hard gluon radiation.

- Cone Radius: The cone radius $R$ used in the jet search has been set equal to 1 .

It is important to stress that the goal of this work is not to find the jet $E_{T}$ scale (as in [49], [50]) but rather to compare the two different methods to reconstruct the jet energy. In the next section the results of this comparison are presented. 


\subsection{2 "Classification" vs. "JTC96"}

As a discriminating variable we have chosen the momentum balancing, definited as

$$
f_{b}=\frac{P_{T}^{J e t}-P_{T}^{\gamma}}{P_{T}^{\gamma}}
$$

We are interested on the width $\sigma_{B}$ of the distribution which quantify the error in the reconstruction of the parton $E_{T}$. The $\sigma_{B}$ depends on $P_{T}^{\gamma}$ and usually the relationship is parametrized as:

$$
\sigma_{B}\left(P_{T}^{\gamma}\right) \sim \frac{\alpha}{\sqrt{P_{T}^{\gamma}}} \oplus b
$$

with $b$ of few percent. In fig. 7.2 the $\sigma_{B}$ is plotted for the two methods.

\section{Photon-Jet balancing}

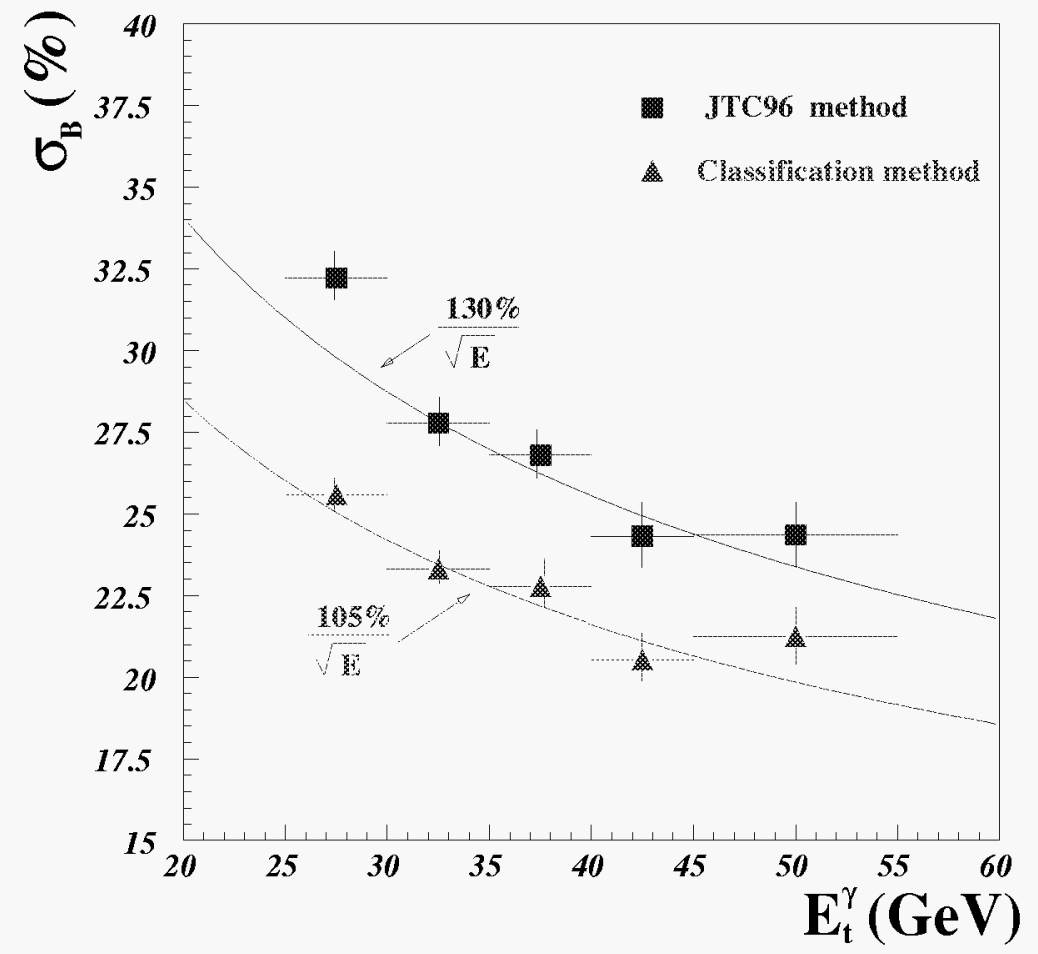

Figure 7.2: Momentum balancing width $\sigma_{B}$ distribution as function of $P_{T}^{P h o}$. Classification and JTC96 methods are compared.

A sensible improvement adopting the "classification method" can be noticed. 
In the next section the Central detector resolution is extracted from data and it will be compared in the two cases. Therefore this method does not depends on resolution effects only but on algorithm effects as well - which have been discussed in Chapter 5 - like gluon radiation, initial state radiation, $k_{T}$ kinks and so on.

\section{4 "Dijet-like" $k_{t}$ Balancing}

In this section we will use an alternative technique to study the jet energy resolution and to extract the contribution due to calorimeter imperfections as being developed by the UA2 collaboration [51]. An imbalance in the jet and photon $\vec{P}_{T}$ s will in a non-null $\vec{k}_{t}$ vector - where $\vec{k}_{t}=\vec{P}_{T}^{j e t}+\vec{P}_{T}^{\gamma}$ - whose components are sensitive to different sources.

\subsection{1 "Dijet-like" $k_{t}$ Description}

Figure 7.3 shows a schematic representation of the transverse momentum vectors of the jet and the photon.

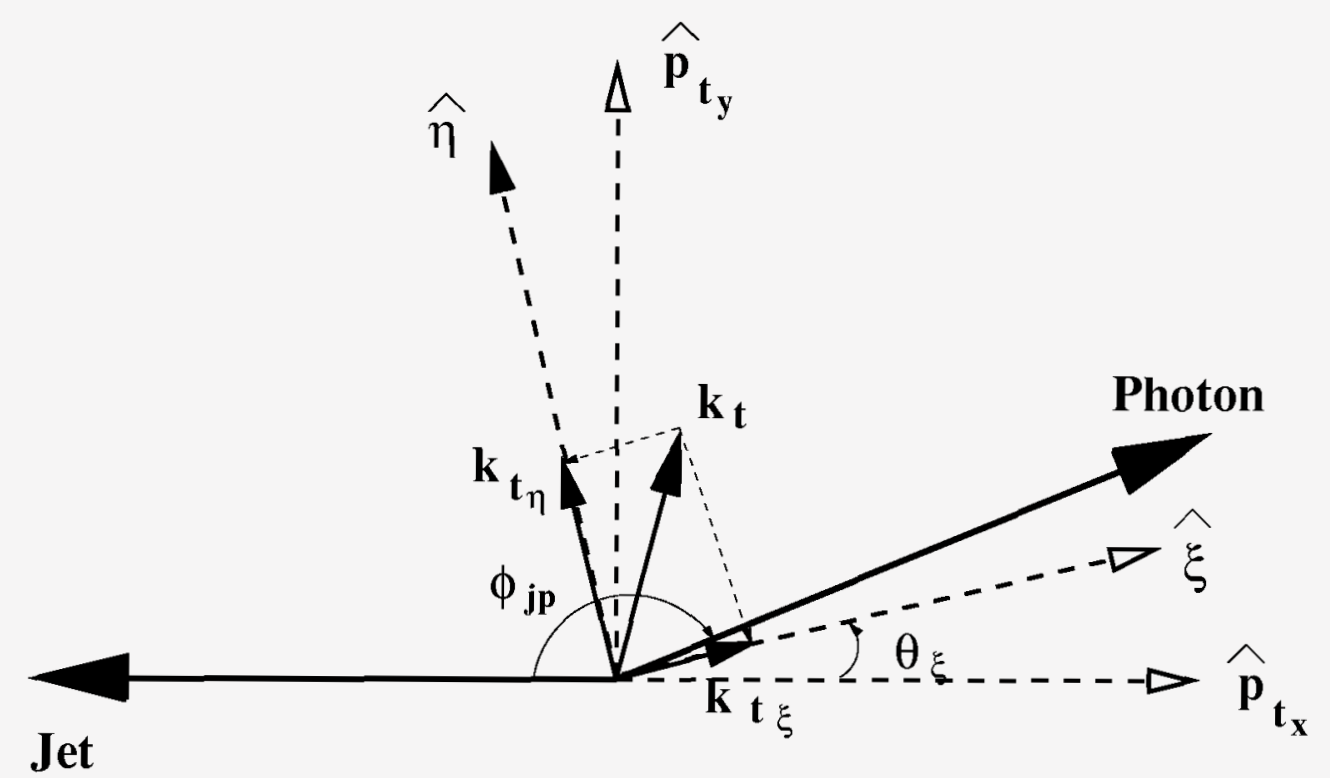

Figure 7.3: $k_{t}$ example: schematic view of a "Photon + Jet" event. The transverse momenta of the jet and photon are shown. They have been decomposed into two components, $k_{t_{\eta}}$ and $k_{t_{\xi}}$. These components are sensitive to different effects responsible for generating the overall $\vec{k}_{t}$ vector.

The jet is chosen to lie along the negative $\mathrm{x}$-axis. The azimuthal angular 
separation of the jet and photon is called $\phi_{j p}$. A pair of orthogonal coordinates have been drawn such that one axis, called the $\eta$-axis ${ }^{2}$, is definited as the azimuthal angular bisector of the photon-jet system. The other axis, the $\xi$-axis, is orthogonal to the $\eta$-axis and defined such that the cross product of their unit vectors points along the z-axis: $\hat{\xi} \times \hat{\eta}=\hat{z}$. The rotation of the $k_{t}$ components from $k_{t_{x}}$ and $k_{t_{y}}$ into $k_{t_{\eta}}$ and $k_{t_{\xi}}$ is described by the angle $\theta_{\xi}$. Since $\phi_{j p}$ is peaked near $180^{\circ} 3$,

the $k_{t_{\xi}}$ component is approximately the difference of the photon and jet transverse momenta, and the $k_{t_{\eta}}$ component is the average transverse momentum times the difference of the azimuthal separation from $180^{\circ}$. The $k_{t_{\xi}}$ component is caused mostly by the energy measurement errors causing transverse energy imbalance and the $k_{t_{\eta}}$ component is caused by angular measurement errors. This can be seen if one consider each of these effects in turn.
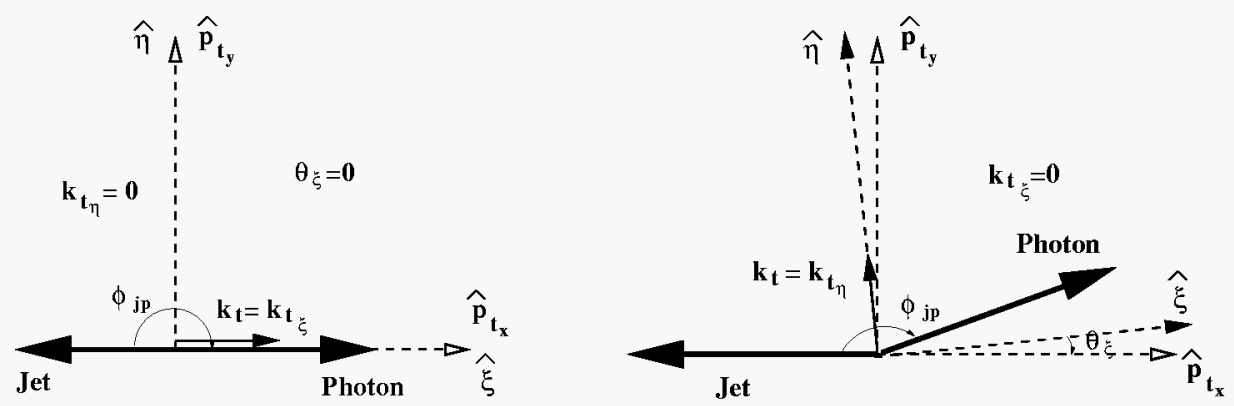

Figure 7.4: a) $k_{t}$ example: jet and photon exactly back-to-back. b) $k_{t}$ example: jet and photon not back-to-back but with equal $P_{T}$.

Consider the case in which $\phi_{p j}=180^{\circ}$ (see fig. 7.4a)). In this case, $k_{t}$ points in the $\hat{\xi}$ direction and the width of the $k_{t_{\xi}}$ distribution reflects the jet energy measurement error, since the photon energy is measured much many precisely ${ }^{4}$

Now consider the other case in which the magnitudes of the transverse momenta of the jets are identical, but the jets are not back-to-back (see

\footnotetext{
${ }^{2}$ It is important to note that the symbol $\eta$ is used here to indicate a component of $k_{t}$ rather then pseudorapidity.

${ }^{3}$ In this approximation one can write:

$$
k_{t_{\eta}} \simeq\left[\left|p_{t}^{j e t}\right|+\left|p_{t}^{\gamma}\right|\right]\left[\frac{\pi-\phi_{j p}}{2}\right] \quad \quad k_{t_{\xi}} \simeq\left|p_{t}^{j e t}\right|-\left|p_{t}^{\gamma}\right|
$$

${ }^{4}$ The electromagnetic cluster is very well measured in the central electromagnetic calorimeter (CEM) with a resolution of $\frac{13.5 \%}{\sqrt{E}}$ compared to about $\frac{105 \%}{\sqrt{E}}$ for jets.
} 
fig. 7.4b) ). It is clear that the $k_{t}$ points in the $\hat{\eta}$ direction and the width of $k_{t_{\eta}}$ gives a measurement of the angular error.

Before studying in more detail the distribution of $k_{t}$ components, we need to understand their relationship with jet variables, namely the dependence of the widths to the jet $E_{t}$.

Starting from the usual definition of transverse energy

$$
E_{t}^{2}=E^{2}-p_{z}^{2}=p_{t}^{2}+m^{2}
$$

Therefore, one may write the difference in $E_{t}$ as :

$$
\begin{aligned}
E_{t}^{j e t}-E_{t}^{\gamma} & =\sqrt{\left(p_{t}^{j e t}\right)^{2}+m_{\text {jet }}^{2}}-p_{t}^{\gamma}=p_{t}^{j e t} \sqrt{\left(1+\frac{m_{\text {jet }}^{2}}{\left(p_{t}^{j e t}\right)^{2}}\right)}-p_{t}^{\gamma} \\
& \simeq p_{t}^{\text {jet }}\left(1+\frac{m_{\text {jet }}^{2}}{2\left(p_{t}^{\text {jet }}\right)^{2}}\right)-p_{t}^{\gamma}+\mathcal{O}\left(\frac{m_{\text {jet }}^{4}}{\left(p_{t}^{\text {jet }}\right)^{4}}\right) \\
& =\left(p_{t}^{j e t}-p_{t}^{\gamma}\right)+\frac{1}{2} \frac{m_{\text {jet }}^{2}}{\left(p_{t}^{\text {jet }}\right)^{2}}+\mathcal{O}\left(\frac{m_{\text {jet }}^{4}}{\left(p_{t}^{\text {jet }}\right)^{4}}\right)
\end{aligned}
$$

Since the ratio of mass ${ }^{5}$ to $p_{t}$ for a jet is roughly $4 \%$, at the leading order we can suppose $p_{t}$ and $E_{t}$ to be identical.

One usually models the calorimeter resolution as:

$$
\frac{\sigma(E)}{E}=\frac{A}{\sqrt{E}}
$$

where $\mathrm{E}$ is the jet energy in $\mathrm{GeV}$. Then, since

$$
E_{t}=E \sin \theta
$$

one can write

$$
\sigma\left(E_{t}\right)=A \sin \theta \sqrt{E}
$$

\footnotetext{
${ }^{5}$ The mass of a jet is not a physically defined object. Usually, it is defined as the invariant mass of the calorimeter tower involved in the cluster treating them as massless particles.
} 
If one assumes independent errors and uses the rms values of $E_{t}$, then

$$
\sigma\left(\left\langle E_{t}^{j e t}-E_{t}^{\gamma}\right\rangle\right)=\sqrt{\left.\sigma^{2}\left(\left\langle E_{t}^{j e t}\right\rangle\right)+\sigma^{2}\left(E_{t}^{\gamma}\right\rangle\right)} \simeq \sigma\left(E_{t}^{j e t}\right)
$$

since $\sigma\left(E_{t}^{\gamma}\right) \ll \sigma\left(E_{t}^{j e t}\right)$.

Remembering that we are dealing with jets in the central detector, $\langle\sin \theta\rangle \sim$ 1 , so

$$
\sigma\left(\left\langle E_{t}^{j e t}-E_{t}^{\gamma}\right\rangle\right)=A \sqrt{E_{t}^{j e t}}
$$

Since $E_{t}^{j e t} \simeq p_{t}^{j e t}$ and of course $E_{t}^{\gamma}=p_{t}^{\gamma}$ one obtains:

$$
\sigma\left(k_{t_{\xi}}\right) \simeq A \sqrt{p_{t}^{j e t}}
$$

One thus expects the width of the $k_{t_{\xi}}$ distribution to grow with square root of the the jet $E_{t}$.

\subsubsection{Origin of the $k_{t}$ Components}

By combining the information on the widths of the $\vec{k}_{t}$ components, $k_{t_{\eta}}$ and $k_{t_{\xi}}$, we can extract the jet energy resolution. Consider the quantities that can be measured and the corresponding sources of $k_{t}$ spread:

- The $k_{t_{\eta}}$ distribution: The emission of gluons as well as the effect of adding or missing other particles - Underlying and Out of Cone energy - will cause a broadening of this distribution. Moreover, the angular error in measuring the jet axis and small relative energy mapping corrections contribute to the spread of the distribution. Finally, hard emission of gluons will increase the width of this component.

- The $k_{t_{\xi}}$ distribution: The calorimeter energy resolution is the main source of the $k_{t_{\xi}}$ component. For jets, one has a collection of particles spanning a range of energies. The resolution on the jet energy is a convolution of the fragmentation properties of the jet with the response of the detector to individual particles. The effects that are described for the $k_{t_{\eta}}$ distribution apply to this components as well, although they may differ in magnitude. Finally systematic offsets in the energy scale will cause a shift in the mean. 


\begin{tabular}{|l|c|}
\hline \hline Component & Sensitive to: \\
\hline$k_{t_{\xi}}$ & Calorimeter energy resolution \\
& QCD gluon emission (other jets) \\
& Misassignement of particles to cluster \\
& $\vec{B}$-field effects \\
\hline$k_{t_{\eta}}$ & Jet angular resolution \\
& QCD hard gluon emission (other jets) \\
& QCD soft gluon emission (other jets) \\
& Misassignment of particles to cluster \\
& $\vec{B}$-field effects \\
& Small scale mapping \\
\hline \hline
\end{tabular}

Table 7.1: Sources of $k_{t}$ components.

The causes of the $k_{t}$ spread are summarized in Table 7.1.

It can be seen that there is a significant overlap in effects which contribute to each of the components. However if one can estimate how the pieces that go into the $k_{t_{\eta}}$ width are related to those same pieces in the $k_{t_{\xi}}$ width, then the energy resolution can be extracted.

\subsubsection{Central Detector Resolution}

In this section we will show how we have extracted the effective detector resolution from the $k_{t_{\eta}}$ and $k_{t_{\xi}}$ widths. Looking at table 7.1 we can learn a number of things from previous studies and from the $k_{t_{\eta}}$ and $k_{t_{\xi}}$ distributions. These are:

- Map scale correction: In [52] is shown that the $\eta-\phi$ distribution of jets in the dijet sample is uniform. In other words this means that relative corrections ${ }^{6}$ work well. The mapping corrections can be ignored to the level of about a percent in the energy resolution.

- Jet angular resolution: The error in the determination of the jet axis is small relative to the width of the $k_{t_{\eta}}$ distribution. An estimation can be made using tracking chamber informations in order to determine the jet axis and comparing them with calorimeter clustering jet axis. The RMS of the mismatch found in [44] was $3^{\circ}$. This kind of error on a 50 $\mathrm{GeV}$ jet gives only a $2.5 \mathrm{GeV}$ contribution to the width of $k_{t_{\eta}}$ which is of the order of $6 \mathrm{GeV}$ (fig. 7.5).

${ }^{6}$ See sec. 4.2 .1 
- Hard gluons: Hard gluon emission can be excluded by putting a cut on the third jet energy. It will be shown later.

- Soft gluon emission: One expects the soft particle effects to be weakly dependent on the hard scattering and hence on jet energy. The hard interaction is a phenomenon that occurs on a short time scale relative to the soft particle effects ${ }^{7}$.

- Other effects: The magnetic field and particle misassignment effects will vary with the fragmentation function of the jets. That function varies logarithmically with energy [52]. Therefore, with a hard cut on the third jet, one expects a weak dependence of $k_{t_{\eta}}$ on energy. The value of the response will depend on how hard one cuts on the third jet since that limits the soft activity.

The widths of the $k_{t_{\eta}}$ and $k_{t_{\xi}}$ distributions, $\sigma_{\eta}$ and $\sigma_{\xi}$, are plotted as a function of $\sqrt{E_{t}^{\gamma}}$. and shown in fig. 7.7.
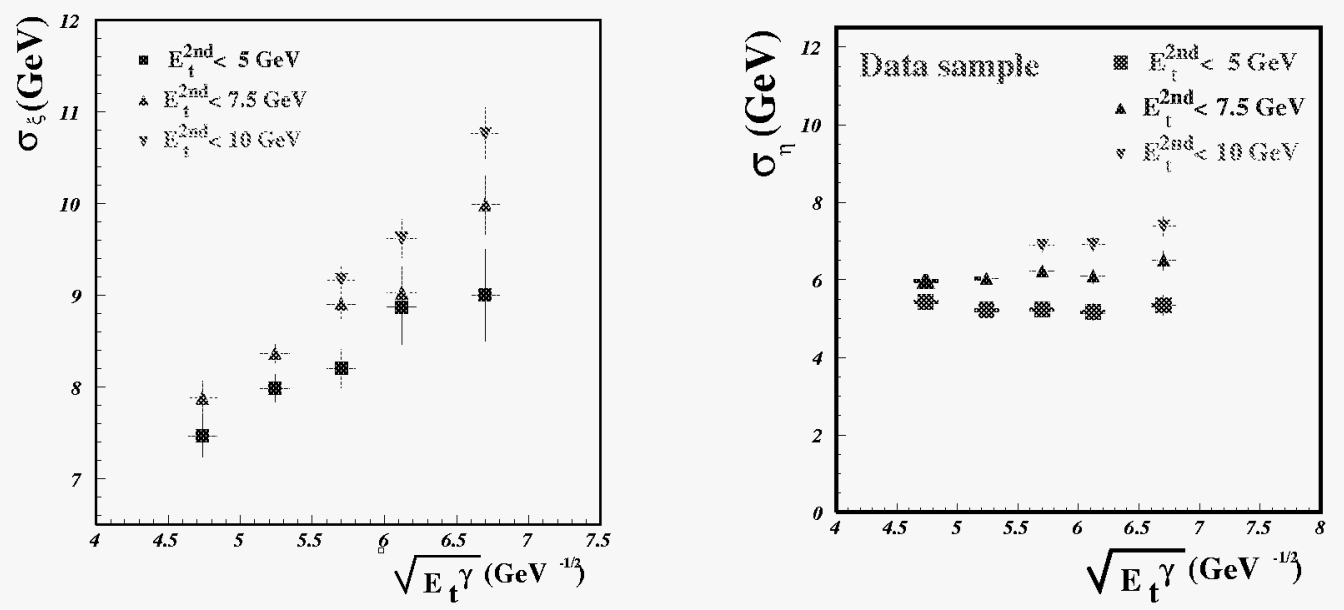

Figure 7.5: Width of $k_{t_{\xi}}$ distribution (a) and of $k_{t_{\eta}}$ distribution (b) as a function of $\sqrt{E_{t}^{\gamma}}$. Three different $2^{\text {nd }}$ jet cuts compared. JTC96 corrections are applied. For the first two bins, where $E_{t}^{\gamma}<35 \mathrm{GeV}$, only the two lower cuts $(5$ and 7.5 GeV) on the $2^{\text {nd }}$ jet are applied.

The cut on the second jet $E_{t_{c u t}}^{2 n d}$ was changed from 5 to $10 \mathrm{GeV}$ in $2.5 \mathrm{GeV}$ steps to investigate the influence of the cut on second jet on the $k_{t}$. Events are selected in the same way as in sec. 7.2 except the $2^{\text {nd }}$ jet cut. There are several clear features:

\footnotetext{
${ }^{7}$ For details see Chapter 1
} 
- The $\sigma_{\xi}$ width depends approximately linearly on $\sqrt{E_{t}^{\gamma}}$. The slopes of the $\sigma_{\xi}$ lines increase with increasing $E_{t_{c u t}}^{2 n d}$. This was expected from previous considerations.

- For all $E_{t_{c u t}}^{2 n d}, \sigma_{\eta}$ has a flat dependence on $\sqrt{E_{t}^{\gamma}}$. The magnitude of $\sigma_{\eta}$ increases with $E_{t_{c u t}}^{2 n d}$. This is what one would expect, the increasing activity in the second jet would add to the overall $\sigma_{\eta}$ level.

Both the $k_{t_{\eta}}$ and the $k_{t_{\xi}}$ distributions are sensitive to soft gluon emission. Question is whether there are identical contributions in both components. We find it plausible that the soft effects be isotropic in azimuth, then the contributions are the same. If this is the case, the soft contribution can be removed by subtracting in quadrature $\sigma_{\eta}$ and $\sigma_{\xi}$. In other words, we are able to subtract the contribution due to finite angle resolution $-\sigma_{\eta}$-from the jet energy resolution $-\sigma_{\xi}$.

$$
\sigma_{D}\left(E_{t}\right)=\sqrt{\sigma_{\xi}^{2}-\sigma_{\eta}^{2}}
$$

$\sigma_{D}\left(E_{t}\right)$ is the effective jet calorimeter resolution.

As shown in fig. 7.6, the $\sigma_{D}$ response curves for the various $E_{t_{c u t}}^{2 n d}$ lie on top of each other. This shows that the factorization of soft effects from the $k_{t_{\xi}}$ component has been successful.

\subsection{4 "Classification" vs. "JTC96"}

The presented results are obtained with a cut on second jet $E_{t_{c u t}}^{2 n d}=5 \mathrm{GeV}$. This choice is motivated by the fact that we want to have a clean sample with a low extra-jet activity and retain a high statistics.

A clear improvement using the "classification method" with respect to the standard "JTC96" method can be seen in fig. 7.7b that shows the $\sigma_{\xi}$ as a function of $E_{T}^{\gamma}$. As expected, the $\sigma_{\eta}$ width is not improved by the "classification method" which cannot recover the angular resolution being due to physics effects mainly.

Finally, in fig. 7.8 the $\sigma_{D}$ value is shown for both methods as a function of $\sqrt{E_{T}^{\gamma}}$.

One can notice the improvement achieved using the "classification method" for all photon energies. A larger improvement is obtained for low $E_{T}^{\gamma}$ as expected. In fact, for low jet energy the jet particles spread out more and fewer mixture towers are present.

In addition, the CTC works better for low energy particles. It was already known that the JTC96 corrections does not work less well for low energetic 


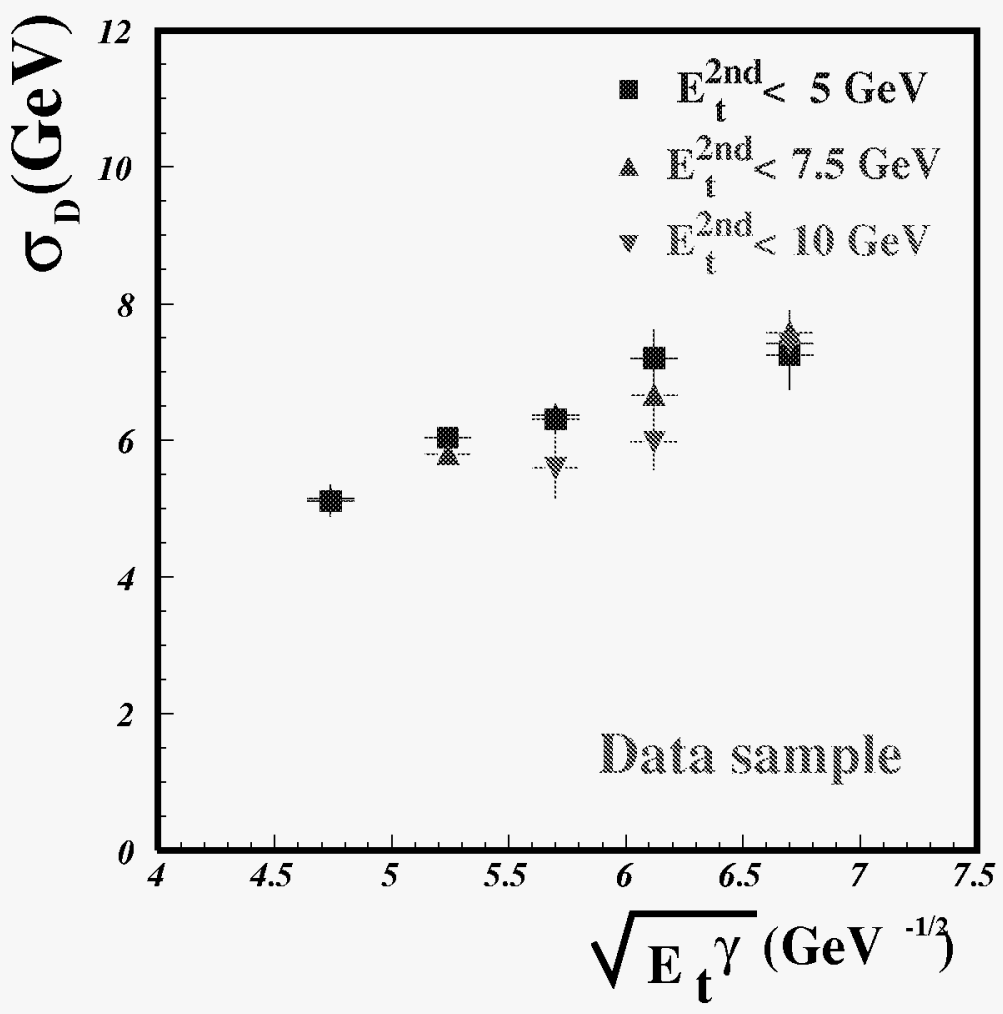

Figure 7.6: Effective detector resolution $\sigma_{D}=\sqrt{\sigma_{\xi}^{2}-\sigma_{\eta}^{2}}$ distribution as function of $\sqrt{E_{t}^{\gamma}}$. Three differents $2^{\text {nd }}$ jet cut are applied and compared. "JTC96 corrections" are applied. For the first two bins where $E_{T}^{\gamma}<35 \mathrm{GeV}$ only two lower cuts on $2^{\text {nd }}$ jet are applied.

jets. We now have a method to cure this problem. The last bin - at $E_{T}^{\gamma} \sim 50$ $\mathrm{GeV}$ - has an anomalous value of $\sigma_{D}$. At that energy we start suffering on statistics. Nevertheless one can again see a significant improvement also in this energy region.

In this sample and after all cuts we can evaluate the Central Detector resolution $\frac{\sigma}{E} \approx \frac{105 \%}{\sqrt{E}}$ using "JTC96 method". Using the new method we reach $\frac{80 \%}{\sqrt{E}}$. It is fair to observe that using together the calorimeter and the tracking information the parametrization of the energy resolution as $-\frac{\alpha}{\sqrt{E}}-$ is not anymore correct. The calorimeter resolution improves with energy whereas the track momentum resolution deteriorates. However, the improvement achieved with the new method is all together evident. 

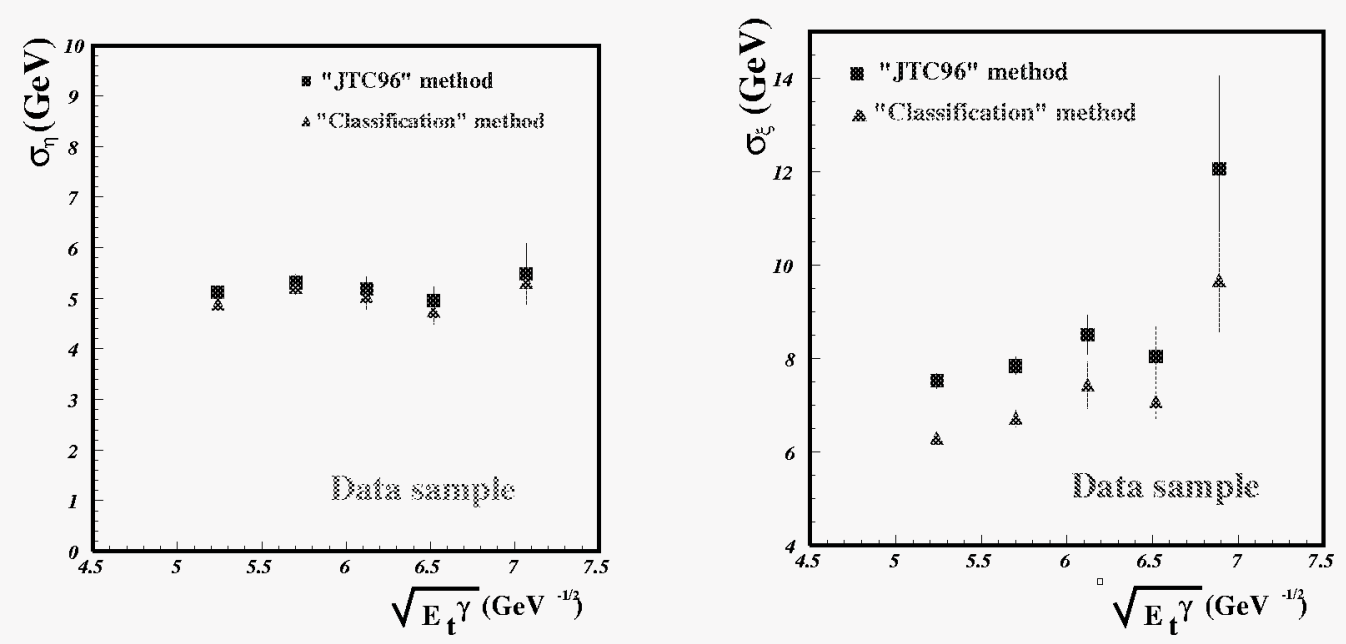

Figure 7.7: The value of $\sigma_{\xi}(a)$ and $\sigma_{\eta}$ (b) as function of $\sqrt{E_{T}^{\gamma}}$ for the two methods. The growing of $\sigma_{\xi}$ and the flat of $\sigma_{\eta}$ with energy is in agreement with what we have previously seen.

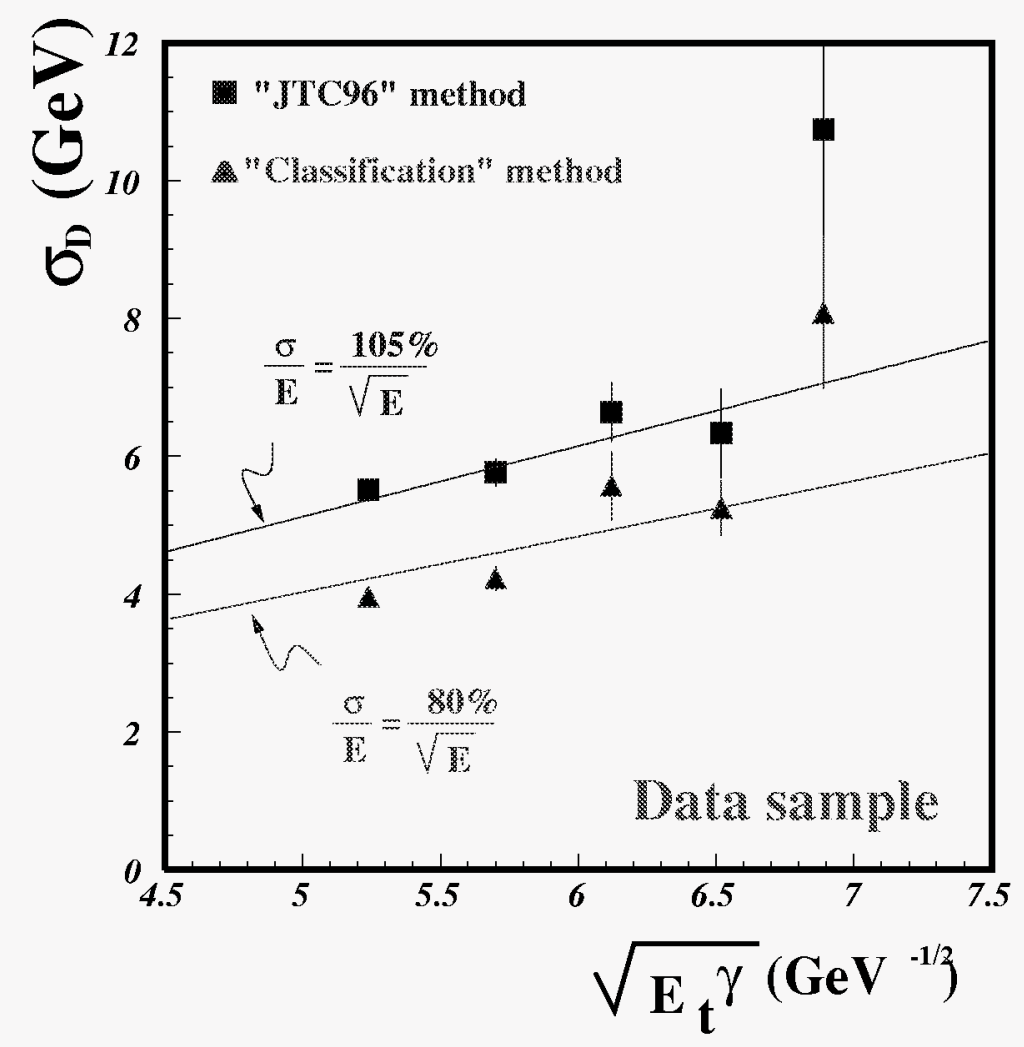

Figure 7.8: The value of $\sigma_{D}$ adopting the two methods as a function of $\sqrt{E_{T}^{\gamma}}$. The two extrapolated resolutions are also shown. 


\section{Conclusions}

In this thesis we first reviewed how jets have been reconstructed by the CDF collaboration in many successful analyses. We recalled how important an improvement in jet energy resolution would be for the physics foreseen in Run II. For example, it was shown in the TeV33 report that a 30\% improvement in resolution would make a large impact in a light Higgs signal significance. Problems in jet energy reconstruction can depend on different effects which we grouped in physics and detector effects. In this thesis these two effects were addressed separately. The impact of physics effects on jet energy resolution was limited by merging extra jets next to a leading jet.

In order to further improve the detector energy resolution a new way of defining the jet energy was proposed. Informations from the CTC and from the strip chambers were used to reconstruct the energy of single particles in the jet whenever possible. The new method, called "classification method", was tested on the photon + jet data sample. The results obtained are encouraging. It was shown how a clear improvement in jet energy resolution can be achieved. The default CDF jet energy resolution can be parametrized as:

$$
\frac{\sigma_{D}}{E}=\frac{105 \%}{\sqrt{E}}
$$

while the resolution of our "Classification" can be expressed as:

$$
\frac{\sigma_{D}}{E}=\frac{80 \%}{\sqrt{E}}
$$




\section{Acknowledgements}

With the several people I met since I started my thesis, it is a mighty task to properly thank all the people with whom I have interacted.

I begin with my advisor, Giorgio Bellettini, who pointed me to the very interesting subjet of jet corrections and put me into the Dijet Mass Group. His assistance in the correction phase was fundamental.

I learned a lot from each member of this gang. At the top there is Steve Kuhlmann to whom saying thanks is not enough. His presence every two weeks - at 8:00am - in videolink and his ability to quickly look into and solve a problem was foundamental in order to keep the work going. I'am especially grateful to him for the huge support provided by e-mail although my personal interpretation of the american language.

Stefano Lami provided invaluable companionship in the early phases and an intellectual support through this year. I will be forever grateful to him for the chance to visit the Rockefeller University and for the shepherd through the most famous places in the New York City (the Webster Hall, the Planet Hollywood ...) and all absolutely free !! Thanks for the generous treatment.

Davide Costanzo and Riccardo Paoletti shared with me any phases of the work encouraging me when the things going wrong and providing, every day, friendship and social support in particular through the "american" summer. It will hard to forgot the memorable trip through the Midwest till New York city (trying to elude cops and their speed traps). They deserve a special thanks for the time (i.e. several days) spent reading the draft of this thesis and remedying my several English errors, being late sometime (thanks Paola and Chiara for the patience).

Stefano Bettelli with his methodical habits taught me how to work with the Analysis Control tool and introduced me in the arcane word of the CDF standard routines. He, as well as "Pippi", gave me a special support in my first interaction with the American English. Without them I still would be in the Chicago O'Hare airport looking for a way to reach Batavia.

I also wish to thank Alessandro, Ernesto, Simone and the summer students for the nice time I had in Fermilab and for the daily excursions to 


\section{Chicago.}

I wish to mention the CDF Pisa group leads by Aldo Menzione which suggestions has been particularly helpful. I thank Paola Giannetti and Sandra Leone for much help and several hints to prepare the final discussion.

Finally I thank my "Pisan" friends who provided a reference point in these years. We have grown up together - sure, we have always eaten the same meal in the canteen... 


\section{Bibliography}

[Standard model, PDF and perturbative QCD]

[1] S. L. Glashow, Nucl. Phys. 22, 579 (1961)

S. Weinberg, Phys. Rev. Lett. 19, 1264 (1967)

[2] P.A.M Dirac

Proceedings of the Royal Society of London, A 114, 243 (1927)

[3] A. Salam

Nobel Symposium 8

edited by N. Svartholm -Stockholm, Almqvist and Wiksell (1968), p. 367.

[4] D.J.Gross and F.Wilczek

Phys. Rev. Lett 19, 1264 (1967)

S.Weinberg

Phys. Rev. D 83633 (1973)

H. Fritzsch, M. Gell-Mann, and H. Leutwyler

Phys. Rev. Lett 30, 1343 (1973)

[5] J.C.Collins, D.E.Soper and G.Sterman

Factorization of hard processes in $Q C D$

in "Perturbative QCD", World Scientific - Singapore

[6] A.D.Martin, R.G.Roberts and W.J.Stirling

Phys. Rev. D. 50 (1994) 6734

[7] B.L.Combridge, J.Kripfganz and J.Ranft

Phys. Rev. D 506734 (1994)

[Hadronization models: indipendent fragmentation]

[8] Ya.I Azimov, Yu.I. Dokshitzer, V.A.Khoze and S.I.Troyan

Phys. Lett. B 165147 (1985)

Z. Phys. C 2765 (1985) 
[9] R.D.Field and R.P.Feymann

Phys. Rev. D 152590 (1977)

Nucl. Phys. B 1381 (1978)

[Hadronization models: String model]

[10] A.Ali et al.

Phys. Lett. B 93155 (1980)

[11] B.Andersson, G.Gustafson, B.Soederberg

Z. Phys. C 20317 (1983)

Nucl. Phys. B 26429 (1986)

B.Andersson, G.Gustafson, G.Ingelman and T.Sjostrand

Phys. Rep. 9733 (1983)

[12] T.Sjostrand

Nucl. Phys. B 248469 (1984)

[13] JADE collaboration, W.Bartel et al.

Phys. Lett. B 157340 (1985)

TPC collaboration, H. Aihara et al.

Z. Phys. C 2831 (1985)

TASSO collabaration, M.Althoff et al.

Z. Phys. C 2929 (1985)

[Hadronization models: cluster model]

[14] D.Amati and G.Veneziano

Phys. Lett. B 8387 (1979)

A.Bassetto, M.Ciafaloni and G.Marchesini

Phys. Lett. B 83207 (1979)

G.Marchesini, L.Trentadue and G.Veneziano

Nucl. Phys. B 181335 (1980)

[15] R.D.Field and S.Wolfram

Nucl. Phys. B 21365 (1983)

[16] B.R. Webber

Nucl. Phys. B 238492 (1984)

[Monte Carlo programs] 
[17] H.-U. Bengtsson and T.Sjostrand

Comp. Phys. Comm. 4643 (1987)

T.Sjostrand

Comp. Phys. Comm. 8274 (1994)

[18] G.Marchesini and B.R.Webber

Nucl. Phys. B 310461 (1988)

G.Marchesini, B.R.Webber, G.Abbendi, I.G.Knowles, M.H.Seymour and L.Stanco

Comp. Phys. Comm. 7115 (1992)

[19] T.Sjostrand

Comp. Phys. Comm. 39347 (1986)

M.Bengtsson and T.Sjostrand

Comp. Phys. Comm. 43367 (1987)

[Top physics at CDF]

[20] F.Abe et al.

Phys. Rev. D50, 2966 (1994)

[21] F.Abe et al.

Phys. Rev. Lett. 73, 225 (1994)

[22] David Gerdes

Prospects for Observing Single Top Production at the Tevatron

$\mathrm{CDF} / \mathrm{ANAL} / \mathrm{TOP} / \mathrm{CDFR} / 2865$

[23] F.Abe et al.

Phys. Rev. D50, 2966 (1994)

[24] F.Abe et al.,

Phys. Rev. Lett. 74, 2626 (1995)

[25] B.Denby, M.Dickson, G.Pauletta N.Wainer

Quark/gluon discrimination at CDF using Neural Networks

$\mathrm{CDF} / \mathrm{DOC} / \mathrm{JET} / \mathrm{CDFR} / 1706$

[26] C.Hill and X.Zang

Fermilab-PUB-94/231-T (1994)

[Higgs physics] 
[27] P. W. Higgs

Phys. Rev. Lett. 12 (1964) 132

[28] CMS Thecnical Proposal

CERN/LHCC/94-38, LHCC/P1 (1994)

[29] ATLAS Thecnical Proposal

CERN/LHCC/94-43, LHCC/P2 (1994)

\section{[Higgs physics at CDF]}

[30] A. Stange, W. Marciano, and W. Willenbrock

Phys. Rev. D49, 1354 (1994); D50, 4491 (1994)

[31] S. Mrenna and G. Kane

CALT-68-1938 (1994)

[32] A. Balyaev, E. Boos and L. Dudko

Mod. Phys. Lett. A10, 25 (1995)

\section{[CDF detector]}

[33] The collider detector at Fermilab

A compilation of articles reprinted from Nuclear Instruments and Methods in Physical Research- $A$

NORTH-HOLLAND - AMSTERDAM

[34] F.Abe et al.

Phys. Rev. D52, 2624 (1995)

[Jet clustering algorithm and jet correction]

[35] Brenna Flaugher, Jim Mueller

A Guide to JETCLU: the CDF Jet Cluster Algorithm

$\mathrm{CDF} / \mathrm{DOC} / \mathrm{JET} / \mathrm{CDFR} / 1814$

F.Abe et al.

Phys. Rev. D45, 1448 (1992)

F.Abe et al.

Phys. Rev. Lett. 73, 225 (1994)

[36] L.Keeble, B.Flaigher

New Jet Correction Function QDJSCO 2.0

CDF/ANAL/JET/CDFR/1513

F.Abe et al.

Phys. Rev. D47, 4857 (1993) 
[37] Nathan Eddy

New Relative Jet Corrections for Run $1 A$ and $1 B$ Data

$\mathrm{CDF} / \mathrm{ANAL} / \mathrm{JET} / \mathrm{CDFR} / 3534$

[38] A.Caner, L.Galtieri, J.Lys, W.Yao,A.Yagil

Jet Corrections for Top Mass Fitting

$\mathrm{CDF} / \mathrm{ANAL} / \mathrm{TOP} / \mathrm{CDFR} / 2469$

$\left[K_{T}\right.$ jet finding algorithm]

[39] M.H.Seymour

Z. Phys. C62 127 (1993)

[Charged particles response in minimum bias events]

[40] S.Behrends, M.D.Shapiro,E.Pare

Single Pion Response in the Central Calorimeter

$\mathrm{CDF} / \mathrm{ANAL} / \mathrm{CAL} / \mathrm{CDFR} / 1066$

[41] S.Kuhlmann et al.

Response of the central calorimeter to low-energy Charged particles

$\mathrm{CDF} / \mathrm{ANAL} / \mathrm{CAL} / \mathrm{CDFR} / 1066$

[42] A.A.Bhatti

Isolated Charged Particle Response in 1992-93 Minimum Bias Data $\mathrm{CDF} / \mathrm{ANAL} / \mathrm{JET} / \mathrm{CDFR} / 2838$

[Jet correction using track informations]

[43] Bob Cousins

On sing CTC Track momenta to improve Jet energy resolutio

CDF/MEMO/JET/PUBLIC/1567

[44] G.Punzi et al.

Charged Track correction to Jets and effect on jet energy resolution

CDF/ANAL/JET/CDFR/1015

[45] S.dell'Agnello

Effect of the charged track correction on jet Energy resolution in 88/89

data

$\mathrm{CDF} / \mathrm{ANAL} / \mathrm{CAL} / \mathrm{CDFR} / 1066$

[46] G.Punzi

Invariant Mass spectrum of Jet-Jet events in Proton-Antiproton Collision at $\sqrt{s}=1.8 \mathrm{TeV}$

Ph.D. Thesis, Scuola normale Superiore, PISA (1990) 


\section{[Test beam CEM results]}

[47] L.Balka et al. The CDF Central Electromagnetic calorimeter Nucl. Instrumen. Meth. A262 272-279 (1988)

[Prompt photon study at CDF]

[48] F.Abe et al.

Phys. Rev. D48, 2998 (1993)

[Jet energy scale study]

[49] S.B. Kim and S.Vejcik

Jet $E_{T}$ scale from momentum balancing in $Z+1$ jet and Photon +1 Jet CDF/ANAL/TOP/CDFR/3504

[50] L.Galtieri, J.Lys

How well do we understand jets in Run I? Study of the Jet Energy Scale for Raw Jet $E_{T} \geq 8 \mathrm{GeV}$ $\mathrm{CDF} / \mathrm{ANAL} / \mathrm{TOP} / \mathrm{CDFR} / 3983$

[Detector resolution determination using $\overrightarrow{k_{t}}$ vector]

[51] UA2 collaboration

Phys. Lett. B 154338 (1985)

[52] R.D.St. Denis

Dijet angular distribution in proton-antiproton Collision at Fermilab Tevatron

Ph.D. Thesis, Harvard University, Cambridge, CA (1988) 